University of Tennessee Health Science Center UTHSC Digital Commons

\title{
Effect of Postmenopausal Hormone Replacement Therapy on Heart Rate Variability
}

Diane Todd Pace

University of Tennessee Health Science Center

Follow this and additional works at: https://dc.uthsc.edu/dissertations

Part of the Nursing Commons

\section{Recommended Citation}

Pace, Diane Todd , "Effect of Postmenopausal Hormone Replacement Therapy on Heart Rate Variability" (1998). Theses and Dissertations (ETD). Paper 188. http://dx.doi.org/10.21007/etd.cghs.1998.0235.

This Dissertation is brought to you for free and open access by the College of Graduate Health Sciences at UTHSC Digital Commons. It has been accepted for inclusion in Theses and Dissertations (ETD) by an authorized administrator of UTHSC Digital Commons. For more information, please contact jwelch30@uthsc.edu. 


\title{
Effect of Postmenopausal Hormone Replacement Therapy on Heart Rate Variability
}

\begin{abstract}
Minimal research has addressed effects of postmenopausal hormone replacement therapy (HRT) on heart rate variability (HRV), a measure of cardiovascular autonomic balance and a predictor of cardiac morbidity/mortality. Using an exploratory, pre-test/post-test design to describe HRV in postmenopausal women (PMW) before/after six weeks of HRT, twenty-four Black $(n=15)$ and White $(n=9)$ women, (mean age $=55 \pm 4$ years), were recruited from two primary care clinics $(n=20)$ and one church population $(n=4)$ in a large urban midsouth community. Cardiovascular risk factors for the sample included chronic illnesses of DM $(n=8), \operatorname{HRT}(n=11)$, both DM and HRT $(n=4)$, smoking $(n=9)$, non-exercise $(n=11)$, and obesity quantified by sample mean BMI (mean=28 \pm 7.5 ). Twenty-four hour Holter monitoring and HRV analyses were done at first visit and following six weeks of oral self-administration of PremPro ${ }^{\text {TM }}$ (estrogen: 0.625 mg; medroxyprogesterone acetate: $2.5 \mathrm{mg}$ ).

PMW demonstrated lower, but normal, HRV mean values than those for healthy female controls $(n=118$; age $=35.6 \pm 11.8$ years), Mean values were within normal limits established for the laboratory. Paired t-tests with significance at $p$ ? .10 demonstrated total group improvement for LowHertz $(p=.06)$ and pNN50 $(p=.10)$. HRV values improved for PMW with selected risk factors, consistently for values of TotalHertz and HighHertz, and, to a lesser extent, for values of PNN50 and rMSSD. Decreases in SDNN and SDANN were unexpected and merit further investigation.

White PMW showed no significant differences before/after HRT ; Black PMW demonstrated significant improvement in TotalHertz $(p=.01)$, LowHertz $(p=.03)$, HighHertz $(p=.01)$, and rMSSD $(p=.05$. Correlational analyses of relationship for pre-/post-test HRV values to CV risk factors demonstrated significant ( $p$ ?.10) relationships across HTN and years of menopause. Pre-/post-test significant differences were found for PMW with HTN for TotalHertz ( $p=.08)$, HighHertz ( $p=.04)$, pNN50 ( $p=.03)$, and rMSSD ( $p=.006)$, and PMW * 9 years menopausal for TotalHertz $(p=.07)$, LowHertz $(p=.01)$, pNN50 $(p=.08)$, and SD $(p=.07)$. Previous research findings suggested that PMW not on estrogen have a higher sympathetic mediation of autonomic function. Blacks and PMW $<55$ years of age, following six-weeks of HRT, demonstrated a mediation in sympathetic/parasympathetic balance in post-test values. Because the study was exploratory with numerous analyses at p-value?.10, findings must be interpreted with caution.
\end{abstract}

\section{Document Type}

Dissertation

\section{Degree Name}

Doctor of Philosophy (PhD)

\section{Program}

Nursing

\section{Research Advisor}

Kay F. Engelhardt

\section{Keywords}

Cardiovascular rish factors, cardiac autonomic function, estrogen, heart, hypertension 


\section{Subject Categories}

Medicine and Health Sciences | Nursing

\section{Comments}

Two year embargo expired June 2000 
EFFECT OF POSTMENOPAUSAL

HORMONE REPLACEMENT THERAPY ON

HEART RATE VARIABILITY

\author{
A Dissertation \\ Presented for \\ The Graduate Studies Council \\ The University of Tennessee, Memphis \\ In Partial Fulfillment \\ Of the Requirements for the Degree \\ Doctor of Philosophy
}

From the University of Tennessee, Memphis

By

Diane Todd Pace

June, 1998 
Copyright (C) Diane Todd Pace, 1998

All rights reserved 


\section{DEDICATION}

Sacrifice is such a small word to describe all that my family has done for me while I have pursued my many dreams. Without their love, support, encouragement, and unselfishness, I could never have achieved the many accomplishments which I have attained in my academic and professional career.

It is therefore with a grateful heart that I dedicate this dissertation to:

Jim, my husband of 25 years, who has been my best friend and most faithful encourager when at times I thought I could not continue-Here's to the next 25 years of loving, sharing, and caring;

and

My beautiful daughters, Sara and Cheryl, who have balanced their needs with my commitments-May you fulfill God's calling in your lives as you achieve your personal, educational, and career goals in Occupational Therapy and Law. My prayer is that God will provide you with a family who will give you the same love and freedom which you have given me so that you may also achieve your dreams;

and to

My wonderful parents, Mr. and Mrs. Norman Todd, who have supported me through many years of school, many achievements, as well as disappointments-I share this accomplishment joyfully and gratefully with you .

To all of you, I express my deepest love and appreciation for always being there to encourage me in so many ways. I am blessed to be your wife, your mother, your daughter. 


\section{ACKNOWLEDGMENTS}

No individual can achieve their goals without the assistance and support of others. For the many people who have knowingly or unknowingly played a role in my life as a teacher, friend, counselor, supporter, I express my deepest appreciation. I have been blessed by the personal commitment of my dissertation committee to helping me achieve this academic accomplishment. To my co-chairs, Dr. Kay Engelhardt and Dr. Donna Hathaway, I gratefully express my appreciation for their guidance during the preparation of this dissertation and throughout my doctoral program. The high standards which they exemplify in their roles as educators, mentors, and researchers have established a firm foundation upon which I will base my research career. They have always been accessible, demonstrating their commitment to students, and have truly proven themselves worthy of the title of role model. I would also like to thank Dr. Ray Ke. His clinical perspective gave me new insight into reproductive health, and his willingness to share of his time in helping prepare our grants gave me the opportunity to gain new skills. I am thankful to Dr. Cheryl Stegbauer for her critique of the conceptual model and her willingness to assist me in achieving a scholarly document and to Dr. Peggy Veeser for her commitment to women's health and insight on menopause. Other faculty who have provided direction in my academic career and to whom I am very thankful are Drs. Carol Panicucci of University of Tennessee, Memphis and Nancy Dayton of Union University who frequently served as advocates and counselors. Without my "Community of Scholars” from whom I learned much about scholarship, teamwork, and camaraderie, I would probably have found myself floundering through this learning process. I extend my heartfelt gratitude to Mona, Jean, Patty, Melissa, Ann, Rebecca, for prompting me with reality checks and overlooking my naiveté (even though they did laugh). 
A special acknowledgment is due to Elizabeth Bradshaw, Executive Director of Memphis FamilyCare Center. Without her support and willingness to allow me flexibility in my work schedule, I could not have attained this degree. To the staff of American Way, my friend and colleague, Sara Cheseldine, and my physician preceptors, Drs. Anthony Hicks, Art Levy, and Eugene Epstein, I express my sincere appreciation. To my patients, I am thankful for their encouragement and willingness to participate in my learning process and to contribute to scientific knowledge.

Additionally, I acknowledge the assistance of Mike Musick, pharmaceutical representative for Wyeth-Ayerst Laboratories, for providing the hormones for the drug intervention, and to the corporation for providing financial support to present the results of this dissertation at two research conferences. I appreciate the commitment of this company toward supporting research in women's health. Finally, it is with great appreciation that I recognize the partial funding for this research through NIH Grant:RO1-NR03871 for the heart rate variability monitoring and analyses and to the College of Medicine, Department of Obstetrics and Gynecology, for completing the hormonal assays. 
ABSTRACT

Minimal research has addressed effects of postmenopausal hormone replacement therapy (HRT) on heart rate variability (HRV), a measure of cardiovascular autonomic balance and a predictor of cardiac morbidity/mortality. Using an exploratory, pre-test/posttest design to describe HRV in postmenopausal women (PMW) before/after six weeks of HRT, twenty-four Black $(n=15)$ and White $(n=9)$ women, (mean age $=55 \pm 4$ years), were recruited from two primary care clinics $(n=20)$ and one church population $(n=4)$ in a large urban midsouth community. Cardiovascular risk factors for the sample included chronic illnesses of DM ( $n=8)$, HRT $(n=11)$, both DM and HRT $(n=4)$, smoking $(n=9)$, nonexercise $(n=11)$, and obesity quantified by sample mean BMI (mean=28 \pm 7.5$)$. Twentyfour hour Holter monitoring and HRV analyses were done at first visit and following six weeks of oral self-administration of PremPro ${ }^{\mathrm{TM}}$ (estrogen: $0.625 \mathrm{mg}$; medroxyprogesterone acetate: $2.5 \mathrm{mg}$ ).

PMW demonstrated lower, but normal, HRV mean values than those for healthy female controls $(n=118$; age $=35.6 \pm 11.8$ years $)$, Mean values were within normal limits established for the laboratory. Paired t-tests with significance at $\mathrm{p} \leq .10$ demonstrated total group improvement for LowHertz $(\mathrm{p}=.06)$ and $\mathrm{pNN} 50(\mathrm{p}=.10)$. HRV values improved for PMW with selected risk factors, consistently for values of TotalHertz and HighHertz, and, to a lesser extent, for values of PNN50 and rMSSD. Decreases in SDNN and SDANN were unexpected and merit further investigation.

White PMW showed no significant differences before/after HRT ; Black PMW demonstrated significant improvement in TotalHertz $(\mathrm{p}=.01)$, LowHertz $(\mathrm{p}=.03)$, HighHertz $(\mathrm{p}=.01)$, and $\mathrm{rMSSD}(\mathrm{p}=.05$. Correlational analyses of relationship for pre/post-test HRV values to $\mathrm{CV}$ risk factors demonstrated significant $(\mathrm{p} \leq .10)$ relationships across HTN and years of menopause. Pre-/post-test significant differences were found for PMW with HTN for TotalHertz ( $\mathrm{p}=.08)$, HighHertz $(\mathrm{p}=.04), \mathrm{pNN} 50(\mathrm{p}=.03)$, and 
rMSSD ( $\mathrm{p}=.006)$, and PMW $\geq 9$ years menopausal for TotalHertz $(\mathrm{p}=.07)$, LowHertz $(\mathrm{p}=.01), \mathrm{pNN} 50(\mathrm{p}=.08)$, and SD $(\mathrm{p}=.07)$. Previous research findings suggested that PMW not on estrogen have a higher sympathetic mediation of autonomic function. Blacks and PMW < 55 years of age, following six-weeks of HRT, demonstrated a mediation in sympathetic/parasympathetic balance in post-test values. Because the study was exploratory with numerous analyses at $\mathrm{p}$-value $\leq .10$, findings must be interpreted with caution. 


\section{TABLE OF CONTENTS}

CHAPTER $\quad$ PAGE

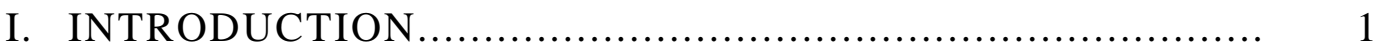

Statement of Problem........................................... 1

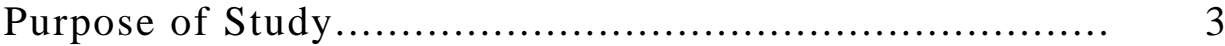

Conceptual Framework....................................... 3

Menopause........................................... 4

Holistic Paradigm.................................... 5

Biomedical Paradigm................................ 6

A Conceptual Model of Menopause..................... 8

Current Research Study.............................. 10

Hormone Replacement Therapy................................ 10

Heart Rate Variability.................................... 12

Measure of Autonomic Cardiac Balance................... 12

A Cyclic Phenomenon.................................... 12

Response to Influencing Factors........................ 13

Predictor of Morbidity and Mortality..................... 14

Measurement Indicators.............................. 14

Summary.............................................. 15

Operational Definitions......................................... 16

Significance of Study ..................................... 20

II. REVIEW OF RELATED LITERATURE............................ 22

Menopause.............................................. 22

Reproductive Hormone Deficiency........................... 22

Physiological Impact of Reproductive Hormone Deficiency.... 24

Cardiovascular Risk Factors................................... 27

Impact of Reproductive Hormone Deficiency on

Cardiovascular Disease................................... $\quad 28$

Hormone Replacement Therapy................................ 30

History ................................................. 30

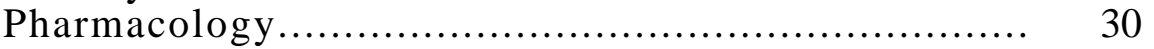

HRT Use and Compliance.................................. 32

Benefits of HRT ........................................... 33

HRT and Breast Cancer....................................... 34

Heart Rate Variability ........................................ 35

Clinical Applications.................................... $\quad 35$

Influences on HRV ...................................... 37

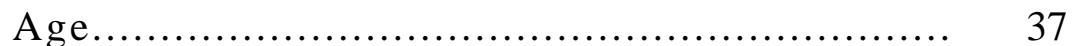

Gender............................................ 38

Pharmacological Interventions......................... 38

Physical Activity....................................... 39

Substance Abuse..................................... 39

HRV and Estrogen....................................... 39

Related Research Studies................................. 41

Summary ............................................. 41 
III. METHODOLOGY ................................................... 43

Research Aims..................................................... 43

Research Design.............................................. 43

Sample And Site................................................. 44

Drug Intervention Protocol.......................................... 45

Instrumentation..........................................................

Demographic............................................. 46

Hormone Assays......................................... 47

Heart Rate Variability .......................................... 47

Procedures..................................................... 49

Recruitment/Enrollment................................... 49

Blood Collection for Hormone Assays........................... 50

Drug Intervention Protocol................................... 50

Heart Rate Variability....................................... 51

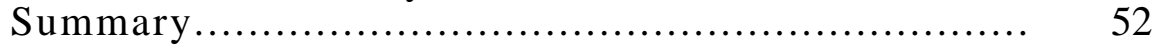

Protection of Human Subjects................................... 52

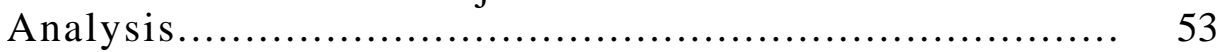

Study Assumptions................................................ 55

Study Limitations............................................. 55

IV. RESULTS ...................................................... 58

Description of Sample.............................................. 59

Demographic Data........................................ 59

Medical/Social History ...................................... 61

Reproductive History ..................................... 64

Physical Characteristics..................................... 66

Cardiovascular Risk Factors.................................... $\quad 70$

Aim One................................................... 71

Pre-Test Heart Rate Variability in PMW............................ 71

Post-Test Heart Rate Variability in PMW ........................ 75

Aim Two....................................................... 78

Aim Three................................................... 81

V. DISCUSSION, RECOMMENDATIONS, AND CONCLUSIONS....... 87

Introduction................................................. 91

Aim One .................................................. 91

Pre-Test Heart Rate Variability in PMW …………........ 92

Post-Test Heart Rate Variability in PMW ....................... 75

Aim Two.................................................... 94

Aim Three................................................... 95

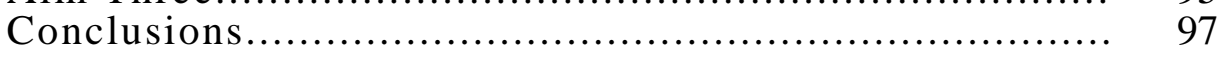

REFERENCES .......................................................... 100 


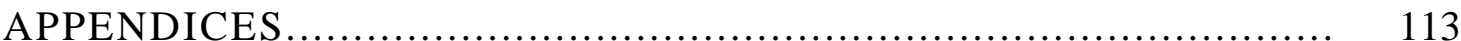

Appendix A: Approval Letters............................. 114

Appendix B: Prescriptive Authority for Principal Investigator..... 118

Appendix C: Study Instruments............................... 120

Appendix D: Protocol for Holter Monitoring...................... 123

Appendix E: Consent Form................................. 129

Appendix F: $\quad$ Drug Documentation Calendar..................... 134

Appendix G: Data Tables: Normative HRV Data for Healthy

Controls....................................... 136

Appendix H: Data Tables: Pre-Test Heart Rate Variability for Postmenopausal Women Across Selected

Cardiovascular Risk Factors.................... 138

Appendix I: Data Tables: Post-Test Heart Rate Variability for Postmenopausal Women Across Selected Cardiovascular Risk Factors..................... 144

Appendix J: Data Tables: Differences Between Pre- and PostTest Heart Rate Variability for Postmenopausal Women Across Selected Cardiovascular Risk

Factors.......................................

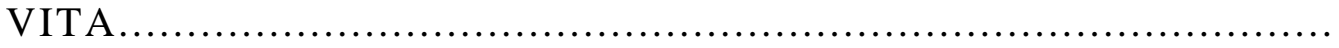




\section{LIST OF TABLES}

\section{TABLE}

PAGE

1 Definitions of Heart Rate Variability................................. 17

2 Characteristics of Postmenopausal Women Across Demographic Variables...

3 Characteristics of Postmenopausal Women Across Medical and Social Variables....

4 Characteristics of Postmenopausal Women Across Reproductive History

5 Mean Height, Weight, Body Mass Index, and Blood Pressure Values of Postmenopausal Women

6 Body Mass Index and Health Risk of Postmenopausal Women....

7 Characteristics of Postmenopausal Women Based Upon the American Heart Association's Cardiovascular Risk Factors

8 Pre-Test Mean Values of Heart Rate Variability in Postmenopausal Women

9 Post-Test Mean Values For Heart Rate Variability in Postmenopausal Women

10 Differences Between Pre- and Post-Test Mean Values For Heart Rate Variability in Postmenopausal Women...

11 Differences Between Pre- and Post-Test Mean Values For Heart Rate Variability in Postmenopausal Women Across Demographic Variable of Race....

12.1 Relationship Between Heart Rate Variability in Postmenopausal Women Across Sample Demographic, Medical/Social, and Reproductive History Variables...

12.2 Comparisons of Differences Across Race for Selected Cardiovascular Risk Factors...

13 Differences Between Pre- and Post-Test Mean Values For Heart Rate Variability in Postmenopausal Women By Absence/Presence of Hypertension.

14 Differences Between Pre- and Post-Test Mean Values For Heart Rate Variability in Postmenopausal Women By Number of Years of Menopause.... 
CHAPTER I

\section{INTRODUCTION}

It is anticipated that by the year 2000 the number of women in the United States living beyond the age of 45 will be 43.5 million (Hargrove \& Eisenberg, 1995; ScharboDehaan, 1996). A naturally occurring phenomenon during this period is the onset of menopause occurring between the ages of $40-58$ with a mean age of 51 . At an increasing life expectancy of 84 years, women may expect to live thirty-three percent (33\%) of their life in this postmenopausal, hypoestrogenic period. In recent years, much scientific data on menopause, mostly from observational, retrospective, and correlational studies, have been published regarding the effects of estrogen deficiency and the apparent protection from morbidity afforded by hormone replacement therapy (HRT). However, definitive answers are still unclear as to the physiological effects of HRT (Eskin, 1994; Gambrell, 1995; Mastrangelo, 1993; Notelovitz, 1989; Porterfield, 1997; Scharbo-Dehaan, 1996). The Women's Health Initiative, a longitudinal, prospective, randomized study funded through the National Institute of Health, is currently being conducted; however, data from this study will not be available until 2005.

\section{Statement of Problem}

Consistent and extensive data from observational studies have indicated that decreased estrogen production is a significant risk factor for cardiovascular disease with coronary heart disease the principal cause of death in postmenopausal women (Newman \& Sullivan, 1996; Gorodeski, 1994). A major source of information is the longitudinal Framingham Heart Study with biennial evaluations of a population which included 2,873 
women. During the 30 year study, 574 women (20\%) developed clinical coronary heart disease with the initial presentation of the disease proving more fatal than for men $(68 \%$ of all coronary deaths compared to $49 \%$ in men). Examination of data from 1600 premenopausal women found only six who had developed coronary heart disease (Castelli, 1994; Newman \& Sullivan). Referring to 1993 American Heart Association statistics, Gorodeski noted that $39 \%$ of women compared to $31 \%$ of men experiencing an acute myocardial infarction die within one year post event . Rosano et al. (1997) documented an imbalance of cardiovascular autonomic control shifting toward sympathetic hyperactivity in menopausal women. According to research results by Kleiger et al. (1987), increased sympathetic activity may predispose an individual to ventricular fibrillation and sudden cardiac death. Currently, estrogen as hormone replacement therapy is increasingly recommended for the prevention and treatment of cardiovascular disease in postmenopausal women with many studies suggesting a 31-35\% reduction in cardiovascular risk (Gupta \& Rymer, 1995; Lip, Beevers, \& Zarifis, 1995; Newman \& Sullivan, 1995; Notelovitz, 1989). Others have criticized many of these past studies for selection bias, however, stating that study participants at onset may have been at lower risk for developing heart disease than the general population due to their having a more favorable life style (Barrett-Connor, 1995; Rebar, 1994).

Heart Rate Variability (HRV), defined as the cyclic changes or fluctuations of heart periods (R-R intervals) over time, has been shown to be a good predictor of cardiovascular morbidity and mortality (Benson, 1995; Cowan, 1995; Kleiger et al., 1987; Saul et al., 1988; Tiller, McCraty, \& Atkinson, 1996; van Ravenswaaij-Arts et al., 1993). However, limited research is available which examines the effect hormone replacement therapy may have on the variability of heart rate, and if this intervention may reduce the risk of cardiovascular disease in postmenopausal women. Research by Huikuri et al (1996), and 
Rosano et al. (1997), have provided only limited data examining this problem, indicating a need for further research.

\section{Purpose of Study}

The purpose of this study was to examine the effect that hormone replacement therapy exerts on cardiovascular autonomic function, as determined by analysis of 24-hour heart rate variability, in postmenopausal women. The following three specific aims will be addressed:

1. Describe heart rate variability in postmenopausal women, ages 45-60.

2. Describe the differences in heart rate variability in postmenopausal women before and after six weeks of hormone replacement therapy.

3. Describe heart rate variability in postmenopausal women relative to selected cardiovascular risk factors before and after six weeks of hormone replacement therapy.

\section{Conceptual Framework}

The conceptual framework for this study is developed around the basic construct, menopause. Defined by the Scientific Group on Research on the Menopause in the 1990's of the World Health Organization (1996), natural menopause is the permanent cessation of menstruation, a consequence of loss of ovarian follicular activity. Menopause occurs with the final menstrual period, followed by 12 consecutive months of amenorrhea with no other attributed pathological or physiological rationale. Because no single independent biological marker for the event exists, menopause can only be identified retrospectively, a year or more after the event. 
Two secondary concepts comprise the framework for this study: hormone replacement therapy (HRT) and heart rate variability (HRV). When viewed from a biomedical perspective, menopause is considered an endocrinopathy resulting in a reproductive hormone deficiency (Utian, 1990). Loss of estrogen often results in a number of physiological effects on the cardiovascular, skeletal, and urogenital systems as well as psychological effects. Hormone replacement therapy is a pharmacological intervention used for therapeutic replacement of hormones which are no longer at physiological levels postmenopause (Rebar, 1994; Voda, 1993). Heart rate variability is defined as the cyclic changes or fluctuations of heart periods (R-R intervals) around the mean heart rate over time (Cowan, 1995). The amount of short and long term variability in heart rate provides a measurement of autonomic nervous system (ANS) balance, delineating between sympathetic and parasympathetic components of autonomic control. Analysis of 24-hour measurements of heart rate variability (HRV) has been shown to be a good predictor of physiological distress and mortality, especially for cardiovascular disease (Benson, 1995; Cowan, 1995; Kleiger et al., 1987; Saul et al., 1988; Tiller et al., 1996; van RavenswaaijArts et al., 1993).

\section{$\underline{\text { Menopause }}$}

Researchers involved in the study of menopause often polarize on their perspective of this phenomenon as being an endocrinopathy or a developmental event occurring during the natural aging process (Voda, 1992). Various theoretical models have been used to describe the concept of menopause. Most scientists agree that the menopausal experience is a complex interaction of both multi-dimensional socio-cultural/psychological factors and biological factors. This primary construct will be examined under two paradigms: holistic and mechanistic/biomedical. 
Holistic Paradigm. The holistic paradigm of menopause describes menopause as intrinsically related to the whole concept of nature and existence and not as an independent, biological entity. It is visualized in a larger system picture and is described well by Hartshorne (1978) as, “composed not of mere matter but of psychical life; pulses of feeling, pains, pleasures....composed also of the experiences of past personal experience, 'memory', and also impersonal memory which is the other portions of the past than one's own previous experiences" (p. 142). Social scientists, Flint and Samil (1990), using a holistic approach for their research, found that within the context of a woman's culture, she learned from childhood, either consciously or unconsciously, how to react to this midlife event.

Researchers who view and describe menopause from this theoretical perspective vigorously argue that menopause is not a disease. They contend that it is a "natural" occurrence during the midlife period of a woman in which she is to be an informed decision maker regarding issues related to this normal developmental phase (Coney, 1994;

Formanek, 1990; MacPherson, 1981; MacPherson, 1995; Voda, 1993). Within this model, menopause is viewed as more than a mechanistic linear happening in the woman's reproductive cycle. Menopause is, instead, a multi-varied, dynamic, interrelated change which has different meanings and experiences for the individual woman. One feminist author noted that women experience menopause differently, some finding the transition as difficult and disabling and others expressing it as positive and easy (Zita, 1993). She defined menopause as a "personal, physical, cultural, and political event, located both inside and outside the physical boundaries of the body and inside a larger body politic" (p. $68)$.

Anthropologists Brown and Kerns (1985) studied the experiences of middle-aged women across a variety of cultures and societies and attempted to develop a transcultural definition of midlife. These researchers were not able to describe a typical menopause. 
Contrary to the negative reports often documented in industrialized cultures, menopause often remains an unreported and unrecognized event in some nonindustrialized societies. Their research showed that for some women this midlife event was often perceived as a positive socio-cultural change. Through menopause, women were often freed from cumbersome restrictions imposed upon them as younger women. The change often allowed them to enter an era of special status and provided them recognition beyond their individual household. Woods (1994), evaluating menopause research from different perspectives indicated that researchers from the holistic perspective, should be challenged to develop an integrative perspective of menopause with studies transcending traditional disciplinary boundaries.

Biomedical Paradigm. Within the biomedical paradigm, the mechanistic philosophy of science is used to explain the mid-life change known as menopause. Changes in the endocrinology of the gonadal-hypothalmic-pituitary feedback mechanism provide a basis for defining menopause as "endocrinopathy", a cause and effect event occurring at one point in reproductive time. Under this paradigm, menopause is considered an objective, biological change resulting from a gradual physiological decline in ovarian activity (BarrettConnor, 1995; Eskin, 1994; Grady et al., 1992; American College of Physicians, 1992; Scharbo-Dehaan, 1996).

Two rationales have been given for endocrinological changes occurring during menopause. One rationale asserts that cessation of ovarian follicle development initiates the hypothalamic-pituitary change, triggering menopause as a result of compromised ovarian function. The alternative view suggests that depletion of the ovarian follicles is actually a consequence of age-related changes in the central nervous system effecting the secretion of gonadotropin-releasing hormone $(\mathrm{GnRH})$, follicle stimulating hormone (FSH) and luteinizing hormone (LH) (Wise, Kranjnak, \& Kashon, 1996). Follicles no longer develop in response to these hormones resulting in loss of negative feedback inhibition and leading 
to markedly decreased levels of sex steroids produced by the ovaries (Porterfield, 1997; Schinfeld, 1994).

Estrogen, progesterone, and androgen are the primary sex steroids secreted by the ovaries. Estrogen is present in three types: estradiol, estriol, estrone. Estradiol is both the most potent and plentiful of ovarian estrogen. At the time of menopause, the serum estradiol level drops to about one sixth the mean level for younger women. Although ovarian production of estradiol does not cease entirely, its primary source at menopause is peripherally from androgens. Estriol is not produced by the ovaries in appreciable amounts except during pregnancy. Estrone, the predominant estrogen in the postmenopausal period, is produced in adipose tissue or produced peripherally from androgens (Porterfield, 1997).

Progesterone is produced in all ovarian endocrine cells and is a precursor for androgens and estrogens. Synthesized normally during the postovulatory phase of the menstrual cycle, its primary function is to transform the uterine endometrium, which has proliferated as a result of estrogenic influence, into a secretory stage. In menopause, it's synthesis is decreased to about one third of that in the follicular phase of younger women with primary production in the adrenal gland. This hormonal loss has important effects on the endometrium and the basis for adding progesterone to estrogen therapy in postmenopausal women with intact uteri (Porterfield, 1997; Schinfeld, 1994).

During the reproductive years, androgens, such as testosterone and androstenedione, are produced by both the adrenal glands and the ovaries. With a loss of ovarian function in menopause, as much as $50 \%$ of androgen production may be lost. Although linked to changes in sexual behavior and attitudes of women, research has not identified the exact influence of androgenic hormones on a woman's libido (Porterfield, 1997; Schinfeld, 1994; Voda, 1993).

Symptoms, such as hot flashes, night sweats, mood changes, dyspareunia, vaginal atrophy, and headaches are often described as commonplace during this period and are 
attributed to hormone deficiencies. It is these symptoms which most often prompt some women to seek medical intervention.

A Conceptual Model of Menopause. Figure 1 presents a model suggesting the relationships and interactions of various influences on the conceptualization of the construct, menopause. External environmental forces such as family, culture, politics, media, education, and economy, as well as the healthcare provider, help to shape each woman's personal beliefs and values associated with the concept of menopause. The openness of the circles suggests that these influences are also shaped and changed by the individual woman once she has conceptualized a concrete image of this phenomenon. The smaller circle represents the healthcare provider within the global perspective of the total environment and provides recognition that this particular influence may be a strong, independent force. Not all cultures, nor all women, will have interaction within this influence; however, this professional often exerts a dominant effect in western cultures. Therefore, healthcare providers have an important obligation to provide unbiased information on menopause, specifically when the conceptualization includes decision making regarding the use of hormone replacement therapy (Rothert, 1990). Two smaller intertwining circles represent the biomedical and holistic philosophical paradigms of menopause. These two major paradigms offer underlying assumptions and information about menopause and serve as filters through which this information is assimilated into a personal conceptualization of menopause. A small area of balance between the two paradigms is represented by overlapping of the circles to illustrate personal resolution of conflicting assumptions and information which have been filtered through the biomedical and holistic paradigms of menopause. The concept, menopause, is represented by a parallelogram which signifies the choices an individual woman makes in the development of her personal conceptualization of the phenomenon in view of the broad influences in the model. 


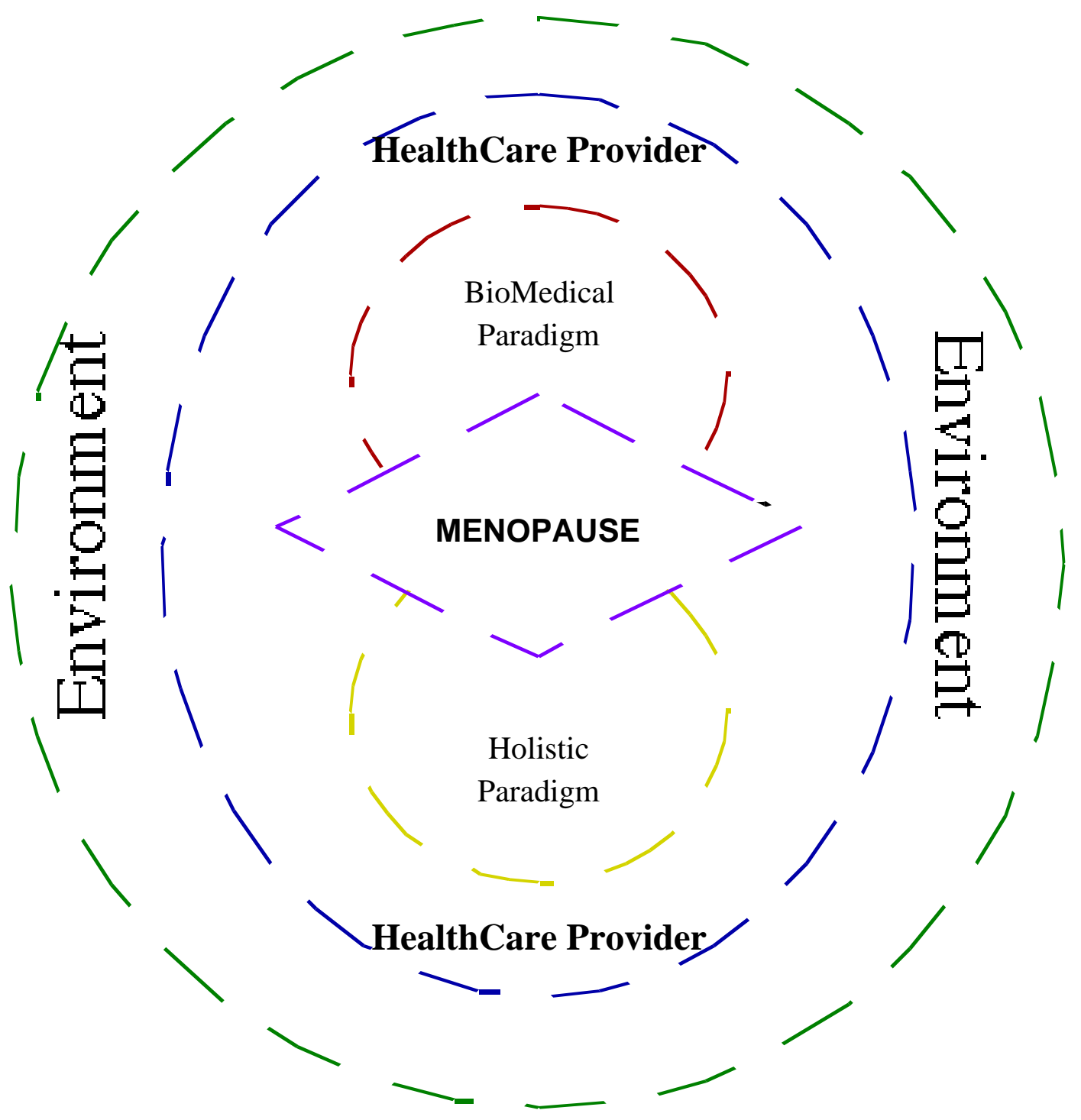

Figure 1. A Model Depictiing the Influences on the Conceptualization of Menopause. 
Current Research Study: A Biomedical Paradigm. Although recognizing that menopause is multi-dimensional, the conceptual framework for this study is developed under the biomedical paradigm. Within this paradigm, menopause is viewed as a reproductive hormone deficiency with women predisposed to cardiovascular disease, osteoporosis, and other morbidity due to decreased estrogen production. Hormone replacement therapy (HRT) is used to reduce symptomatology, prevent pathology, and protect the woman from an accelerated aging process related to estrogen deficiency. This study is conceptually focused on the effect this reproductive hormone deficiency has on the cardiovascular system. From a mechanistic/biomedical perspective, it is conceptually delineated by the effect of reproductive hormone deficiency and hormone replacement on cardiac autonomic function (CAF). Quantification of CAF can be further described through the measurement and analysis of heart rate variability.

\section{Hormone Replacement Therapy}

With loss of ovarian function resulting in a decrease in estrogen levels, proponents of the biomedical paradigm promote the use of HRT to reduce or reverse the negative effects caused by this deficiency. The American College of Physicians (1992) recommend in their "Guidelines for Counseling Postmenopausal Women about Preventive Hormone Therapy" that all women regardless of ethnicity consider taking hormone replacement therapy as a prevention for disease and to prolong life. For postmenopausal women with intact uteri, two regimens of therapy are suggested for clinical use: (a) estrogen $(0.625 \mathrm{mg})$ plus cyclic progestin $(5-10 \mathrm{mg})$ for 10 to 14 days per month; or (b) estrogen (0.625 mg) and continuous progestin $(2.5 \mathrm{mg})$ per day.

Controversy, however, exists regarding the use of HRT with feminist researchers and clinicians questioning results from studies on use, risks, and benefits of replacing artificial hormones at pharmacological doses (MacPherson, 1993; Voda, 1993). Their 
belief is that transition to menopause is gradual, and menopause is a natural life changing process, not a disease process. Under a holistic paradigm as their conceptual framework for menopause, administration of hormone replacement therapy is illogical, unhealthy, and unethical. The response to menopausal changes under this framework is to teach women how to live their lives in a more healthy manner. They further advocate that information on risks and benefits be shared with women in an unbiased manner by healthcare providers in order to permit the women to make an informed choice on use of HRT (Logothetis, 1993; MacPherson, 1993).

Researchers who practice under the biomedical paradigm also express some concern about the potential side effects and risks which a woman may incur as a result of taking HRT. Links to breast cancer have raised serious questions about the safety of HRT even though its cardioprotective or osteoporosis prevention benefits have been lauded (Barrett-Connor, 1995; Colditz, Hankinson, \& Hunter, 1995; Ettinger, Quesenberry, Schroeder, \& Friedman, 1997). These researchers, although promoting HRT as an intervention to improve quality of life for the postmenopausal woman, advocate that the risks of HRT be clearly delineated and that research continue to develop alternative approaches to therapy which would provide similar benefits (Rebar, 1994).

Both paradigms are important when examining the concept of hormone replacement therapy. Pathological disorders (osteoporosis, cardiovascular disease) affecting postmenopausal women have been associated with estrogen deficiency with some women having higher risk factors for these diseases. Nonetheless, HRT is also an artificial measure to change estrogen deficiency, and risks are apparent. The essential quality surrounding the concept of HRT is the right of women to receive unbiased information and to make an informed personal choice on use of HRT based on that information. 
$\underline{\text { Heart Rate Variability }}$

The concept of heart rate variability (HRV) is also discussed under a biomedical framework. HRV is the measurement of variation of one cardiac cycle to another over time (Keehn, 1992).

Measure of Cardiac Autonomic Balance. Heart rate variability is defined as cyclic changes or fluctuations of heart periods (R-R intervals) around the mean heart rate over time. The amount of short and long term variability in heart rate provides a measurement of autonomic nervous system (ANS) balance. The cyclic fluctuations in the ANS balance create the variability of the heart waveforms and delineate between sympathetic and parasympathetic components of autonomic control (Cowan, 1995; van Ravenswaaij-Arts et al., 1993).

HRV has been utilized in scientific research to characterize the cardiac control mechanism of the ANS (Keehn, 1992). Controlling vital functions such as cardiac action, temperature regulation, metabolism, digestion, fluid balance, and excretion, the ANS has a significant effect on total body homeostasis. The ANS is divided into two major divisions, sympathetic and parasympathetic. Activation of the sympathetic division results in cardiac responses of vasoconstriction, increased force and rate of cardiac contractility, and increased conduction velocity in the myocardium. Aptly described by the phrase "fight or flight", the sympathetic nervous system prepares the body for responding to emergencies. Exerting an antagonistic response to sympathetic function, the parasympathetic division of the ANS induces selective vasodilation of blood vessels and decreases the rate and force of cardiac contractility (Keehn, 1992; Spencer, Nichols, Lipkin, Henderson, \& West, 1993).

A Cyclic Phenomenon. Continuous cycling is a positive characteristic of the autonomic nervous system. Measurement of this cycling produces the frequency parameters which occur in HRV. The length of time in which one cycle occurs is called a period. Keehn (1992) relates this concept to certain mechanistic happenings: (a) the earth's 
rotation of one day is composed of 86,400 seconds; (b) the average human breath is about four seconds; (c) the average heart beat is 0.83 seconds; and, (d) the vibration of middle $\mathrm{C}$ on a piano string is approximately 0.00379 seconds.

Keehn (1992) further discusses cyclic phenomenon as expressed in frequency, the number of cycles which occur in a specific period of time. Named after German physicist, Heinrich Hertz (1857-1894), a unit of frequency is called "hertz" (Hz.). One unit of frequency is equal to one cycle per second. "With this nomenclature, the earth rotates at $0.000011574 \mathrm{~Hz}$., middle $\mathrm{C}$ has a frequency of $264 \mathrm{~Hz}$, an average heart rate is $1.2 \mathrm{~Hz}$, and respiration has an average frequency of $0.25 \mathrm{~Hz} "($ p. 8).

Because the heart rate is not constant but is a composite of multiple frequency components, HRV is characterized by discrete frequencies. Keehn (1992) further describes the analysis of HRV by comparing it to a prism. Similar to the white light which enters a prism and is separated into a spectrum of differentiated colors, HRV is separated by the mathematical analysis called Fast Fourier Transformation into characteristic frequencies. These frequencies are then used to evaluate the function of the autonomic nervous system.

$\underline{\text { Response to Influencing Factors. Endogenous and exogenous factors can influence }}$ changes within the ANS leading to alteration of HRV responses. Marked variability among individuals result from physical, psychological, environmental, or pharmaceutical effects, as well as from variation in instrumentation or measurement of values. Various researchers have noted the influence of age, race, and gender on variation of ANS function, with lower autonomic function (AF) values identified in women, Blacks, and elderly (Braune, 1996; Cowan, Pike, \& Burr, 1994; Liao, 1995; Pace, Engelhardt \& Hathaway, 1997). Age has been implicated in some studies as a cause for decreasing resting heart rate variation, suggesting a lowered vagal tone in elderly participants (van Ravenswaaij-Arts et al., 1993). Body size and smoking status (Kageyama et al., 1997), hormone levels (Rosano, 1997), exercise (Davy, 1996), and medications (Cowan, Pike, Burr, Cain, \& Narayanan, 1993) 
have also been implicated as influencing AF. Additionally, various diseases such as hypertension, heart failure, cardiac arrhythmias, and diabetes mellitus may also affect results of HRV (van Ravenswaaij-Arts et al., 1993).

In addition to physiological factors, psychological factors are implicated in ANS modulation. Mental stress evokes an increase in blood pressure and heart rate secondary to an increase in sympathetic activity and a decrease in parasympathetic activity (Low, 1993; Tiller et al., 1996). Research conducted by Bonnet and Arand (1997) and Chokroverty (1993) documented diurnal changes or periodic changes during day and night periods on HRV. Their research found autonomic alterations, primarily within the cardiovascular and respiratory systems, during normal sleep. In non-REM sleep, a decrease in heart rate and blood pressure was noted. Further decrease was noted during REM sleep with a predominance of parasympathetic activity.

Predictor of Morbidity and Mortality. Analysis of 24 hour measurements of heart rate variability (HRV) has been shown to be a good predictor of physiological distress and mortality, especially for cardiovascular disease (Kleiger et al., 1987; Marquette Electronics, 1990; Saul et al., 1988; van Ravenswaaij-Arts et al., 1993). A sympathovagal imbalance in coronary artery disease and hypertension may be detectable with HRV analysis. Low heart rate variability has been associated with an increased risk for sudden cardiac death in patients with diabetes and in those who have experienced a myocardial infarction.

Measurement Indicators. The non-invasive clinical method for assessing measurement of HRV is conducted by 24-hour Holter monitoring which provides a continuous recording of electrocardiographic data. Analysis of the data occurs across two domains, frequency and time (Cowan, 1995; Marquette Electronics, 1990). Frequency domain data are mediated by the sympathetic and parasympathetic nervous system control and are reported by the values of Total Hertz, High Hertz, Low Hertz, and Ratio of Low to High Hertz. Time domain data are a general measure of autonomic nervous system balance. 
Circadian function is represented by the values of SDNN, SDANN, and SD.

Parasympathetic response by vagal influence is represented by pNN50, and rMSSD.

Individual terms are further defined in the section, Operational Definitions and in Table 1.

\section{Summary}

According to Jacox (1974) concepts are "abstract representations of reality" (p. 351) and must be defined precisely to prevent confusion and improve communication between researchers. The construct of menopause, and the concepts of hormone replacement therapy and heart rate variability, have both independent meanings and, within this study, interdependent meanings.

Menopause is visualized as the highest abstraction of the concepts. Although multidimensional and influenced by many endogenous and exogenous factors, when filtered through a biomedical paradigm, menopause is perceived as a reproductive hormone deficiency. This deficiency affects many metabolic and physiological functions within a woman's body, including the cardiovascular system. Research has shown that these effects can be altered by administering hormone replacement therapy, a pharmacological intervention consisting of estrogen and progesterone.

The effect of HRT on the cardiovascular system has been investigated significantly over the last decade, but little research has been done to investigate its effect on the third concept, heart rate variability. Defined as cyclic changes or fluctuations of heart periods around the mean heart rate over time, HRV has been used as a quantitative measurement of cardiac autonomic balance and a predictor of cardiac morbidity and mortality. Similar to the construct, menopause, HRV is also influenced by many exogenous and endogenous factors. This study will investigate the relationship between the concepts of HRT and HRV in postmenopausal women. 
Operational Definitions

1. Women who were postmenopause were operationally defined as amenorrheic for 12 months; FSH level $\geq 30 \mathrm{mIU} / \mathrm{ml}$; serum estradiol level $\leq 30 \mathrm{pg} / \mathrm{ml}$ (Speroff, 1994).

2. Hormone replacement therapy was operationally defined as the pharmacological replacement of estrogen $(0.625 \mathrm{mg})$ and progesterone $(2.5 \mathrm{mg})$. The hormones were administered in a single, oral caplet developed and distributed by the pharmaceutical company, Wyeth-Ayerst Laboratories as PremPro ${ }^{\mathrm{TM}}$ and dispensed by the primary investigator, a certified Family Nurse Practitioner.

3. Heart rate variability was operationally defined by four frequency domain values: Total Hertz, Low Hertz, High Hertz, and Low/High Ratio; and by 5 time domain values: pNN50, rMSSD, SD, SDNN, and SDANN. See Table 1 for further delineation of each variable. 
Table 1

Summary of Heart Rate Variability Measurements

\begin{tabular}{|c|c|c|c|c|}
\hline Variable & Definition $^{1}$ & Normal & $\begin{array}{c}\text { Values } \\
\text { Borderline } \\
\end{array}$ & Abnormal \\
\hline \multicolumn{5}{|c|}{ Frequency Domain Measures } \\
\hline \multirow[t]{2}{*}{$\begin{array}{l}\text { Total } \\
\text { Hertz }\end{array}$} & $\begin{array}{l}\text { Standard deviation from all } \\
\text { frequencies }(0.01-1.00 \text { Hertz) over the } \\
\text { total test duration of } 24 \text { hours. }\end{array}$ & $\geq 6.11$ & $5.95-6.10$ & $\leq 5.94$ \\
\hline & $\begin{array}{l}\text { Represents combined sympathetic and } \\
\text { parasympathetic autonomic response. }\end{array}$ & & & \\
\hline \multirow[t]{3}{*}{ Low Hertz } & $\begin{array}{l}\text { Standard deviation from frequencies } \\
\text { between } 0.04-0.15 \text { Hertz. }\end{array}$ & $\geq 5.22$ & $4.97-5.21$ & $\leq 4.96$ \\
\hline & $\begin{array}{l}\text { Represents primarily sympathetic } \\
\text { autonomic response (including blood } \\
\text { pressure modulation) with influence } \\
\text { from parasympathetic activity. }\end{array}$ & & & \\
\hline & Predominant during waking hours. & & & \\
\hline \multirow[t]{3}{*}{$\begin{array}{l}\text { High } \\
\text { Hertz }\end{array}$} & $\begin{array}{l}\text { Standard deviation from frequencies } \\
\text { between } 0.15-0.40 \text { Hertz. }\end{array}$ & $\geq 3.45$ & $3.29-3.44$ & $\leq 3.28$ \\
\hline & $\begin{array}{l}\text { Represents primarily parasympathetic } \\
\text { autonomic response (including } \\
\text { respiratory modulation). }\end{array}$ & & & \\
\hline & Predominant during sleep hours. & & & \\
\hline \multirow[t]{3}{*}{$\begin{array}{l}\text { Low/High } \\
\text { Ratio }\end{array}$} & $\begin{array}{l}\text { Represents the relationship of low to } \\
\text { high frequencies over the total test } \\
\text { duration of } 24 \text { hours. }\end{array}$ & 1.2 & & \\
\hline & $\begin{array}{l}\text { Provides a quantification of balance } \\
\text { between sympathetic and } \\
\text { parasympathetic activity. }\end{array}$ & & & \\
\hline & $\begin{array}{l}\text { Higher ratios reflect primarily } \\
\text { sympathetic autonomic response. } \\
\text { Ratios which achieve a lower value, } \\
\text { close to } 1.2 \text {, reflect a balance of } \\
\text { sympathetic and parasympathetic } \\
\text { activity. }\end{array}$ & & & \\
\hline
\end{tabular}


Table 1 (continued)

\begin{tabular}{|c|c|c|c|c|}
\hline Variable & Definition $^{1}$ & Normal & $\begin{array}{c}\text { Values }^{2} \\
\text { Borderline }\end{array}$ & $\begin{array}{c}\text { Abnorma } \\
1 \\
\end{array}$ \\
\hline \multicolumn{5}{|c|}{ Time Domain Measures } \\
\hline \multirow[t]{2}{*}{ pNN50 } & $\begin{array}{l}\text { Proportion of all adjacent R-R } \\
\text { intervals varying by more than } 50 \\
\text { msec during the total test duration of } \\
24 \text { hours. }\end{array}$ & $\geq 0.6$ & $0.4-0.5$ & $\leq 0.3$ \\
\hline & $\begin{array}{l}\text { Most sensitive to high frequency } \\
\text { components and independent of } \\
\text { circadian influence. Represents vagal } \\
\text { mediation of heart rate and } \\
\text { parasympathetic activity. }\end{array}$ & & & \\
\hline \multirow[t]{2}{*}{ rMSSD } & $\begin{array}{l}\text { Mathematical computation of the } \\
\text { square root of the mean of the sum of } \\
\text { squares of differences between } \\
\text { adjacent R-R intervals. }\end{array}$ & $\geq 14$ & 13 & $\leq 12$ \\
\hline & $\begin{array}{l}\text { Most sensitive to high frequency } \\
\text { components and independent of } \\
\text { circadian influence. Represents vagal } \\
\text { mediation of heart rate and } \\
\text { parasympathetic activity. }\end{array}$ & & & \\
\hline \multirow[t]{2}{*}{ SD } & $\begin{array}{l}\text { Mean of the standard deviations } \\
\text { computed for each 5-minute block }\end{array}$ & $\geq 33$ & $31-32$ & $\leq 30$ \\
\hline & $\begin{array}{l}\text { Sensitive to variability within the } 5 \text { - } \\
\text { minute block and higher frequency } \\
\text { components. Disregards variability } \\
\text { which occurs over longer periods. }\end{array}$ & & & \\
\hline \multirow[t]{2}{*}{ SDNN } & $\begin{array}{l}\text { Standard deviation of all R-R intervals } \\
\text { over the total test duration of } 24 \\
\text { hours. }\end{array}$ & $\geq 85$ & $79-84$ & $\leq 78$ \\
\hline & Sensitive to all sources of variation . & & & \\
\hline
\end{tabular}

(table continues) 
Table 1 (continued)

\begin{tabular}{lllll}
\hline Variable & \multicolumn{1}{c}{ Definition } & Normal & $\begin{array}{c}\text { Values } \\
\text { Borderline }\end{array}$ & $\begin{array}{c}\text { Abnorma } \\
1\end{array}$ \\
\hline SDANN & $\begin{array}{l}\text { Standard deviation of all the means of } \\
\text { R-R intervals from each 5-minute block } \\
\text { and computed over the total test } \\
\text { duration of 24 hours. }\end{array}$ & $\geq 69$ & $66-68$ & $\leq 65$ \\
& $\begin{array}{l}\text { Differentiates how much the heart rate } \\
\text { differs during each 5-minute period } \\
\text { from the mean heart rate over 24 hours. } \\
\text { Sensitive to low frequencies and } \\
\text { variations due to posture and activity. }\end{array}$ & & \\
\end{tabular}

${ }^{1}$ Source: Marquette Electronics, (1990). Heart rate variability: Physician's Guide.

Marquette Electronics: Milwaukee, WI. Marquette Electronics (1990).

${ }^{2}$ Source:Cashion, A. (1998). Twenty-four hour heart rate variability measures in healthy controls. Unpublished raw data, University of Tennessee, Memphis.

Note. Sample $N=158$, mean age $=36.2 \pm 11.2$, range: $18-65$ years; Men=40 $(25 \%)$, Women $=118(75 \%) ;$ Blacks=29 $(19 \%) ;$ Whites $=121(77 \%)$; Other $=7(4 \%)$. 
Significance of Study

With average age expectancy increasing, American women can expect to spend greater than one-third of their lives post-menopausal in a hypoestrogenic state. Based on the 1990 U.S. Census data, there are over 40 million women who are $\geq 50$ years and who have had or will soon have their last menstrual period (Scharbo-Dehaan, 1996). The effect of this hypoestrogenic state on molecular and physiological changes in health status has been a focus of many research studies over the last decade (Rebar, 1994). These studies have especially shown a relationship between cardiovascular disease and decreased estrogen production.

In studying the effect of estrogen on cardiovascular disease, many studies have reported a positive relationship on lipid levels and a beneficial effect on glucose metabolism (Gupta \& Rymer, 1996; Lip, Beevers, \& Zarifis, 1995), improved endothelial function through its antioxidant activity (Koh et al., 1997), and improved hemodynamics (Lip et al., 1995; Rebar, 1994). Morbidity and mortality statistics published in the United States and Great Britain have shown that in women above 50 "the cumulative absolute risk of dying from cardiovascular disease is 31\%" (Gupta \& Rymer, 1996, p. 119). In an analysis of studies on estrogen and the risk of heart disease, Rebar found significant evidence which strongly supported the view that postmenopausal estrogen therapy substantially reduced the risk of coronary heart disease. At the 10 year followup of the prospective Nurses' Health Study, researchers concluded that estrogen use was associated with a significant (up to $50 \%$ ) reduction in coronary heart disease morbidity and mortality (Stampfer \& Golditz, 1991).

In a review of literature, two studies were found which examined the effect of estrogen on HRV. Huikuri et al. (1996) and Rosano et al. (1997) both found higher heart rate variability values in postmenopausal women on HRT. Empirical evidence has shown 
that estrogen positively affected the cardiovascular system, and measurement of heart rate variability was considered to be a good predictor of cardiovascular status. Therefore the significance of this study was to add further empirical data to the limited body of scientific knowledge regarding the effect of hormone replacement therapy on HRV. 
CHAPTER II

\section{REVIEW OF RELATED LITERATURE}

Menopause is a universal phenomenon in the reproductive/developmental life cycle of a women. As previously discussed under the conceptual framework, there are sociocultural/psychological changes which influence a woman's transition to this period of life and physiological changes which occur as a result of declining ovarian function and aging. This study examines only the physiological change in the cardiovascular system as measured by heart rate variability and variations in $\mathrm{HRV}$ which occur in response to postmenopausal hormone replacement therapy.

Because this study was conducted within a conceptual framework of a biomedical paradigm, the review of literature will address the research relevant to scientific knowledge produced under this same paradigm. Additionally, the two concepts of hormone replacement therapy and heart rate variability have been incorporated within the same frame of reference.

\section{Menopause}

Under the biomedical paradigm, menopause is defined as a state of reproductive hormone deficiency. It is characterized as a period of biological and chemical changes which occur in response to the normal aging process.

\section{$\underline{\text { Reproductive Hormone Deficiency }}$}

Scientists studying the physiological changes in the postmenopause period which result in decrease of reproductive hormones, have categorized menopause as an 
endocrinopathy (Utian, 1990). Morphologic changes identified in postmortem studies, such as a decrease in the number of ovarian follicles, occur as genetically pre-programmed events. Additionally, examination found ovaries to be fibrotic and, occasionally, hyperplastic and hypertrophic (Krouse, 1994; Utian, 1990).

Manifestations of the decrease in ovarian response during menopause include a shortening of the follicular phase, cessation of ovulation, and ultimate amenorrhea. Estrogen production changes from the primary form of estradiol-17ß produced premenopausally to estrone, a weaker estrogen formed from peripheral conversion of estradiol or androstenedione and also produced primarily in the adipose tissue. Whereas premenopausal women will produce 50-500 micrograms of estradiol daily during the first half of a menstrual cycle, production of less than $30 \mathrm{pg} / \mathrm{ml}$ documents menopausal status. Only 15-100 micrograms of the naturally produced estrogen, estrone, are produced daily by postmenopausal women. Follicle stimulating hormone (FSH) produced by the pituitary gland and important in the negative feedback mechanism of ovulatory control premenopausally, increases and remains at high level (> 30 IU/L) in menopause because the ovary no longer responds to its stimulatory efforts. Additionally, receptor tissues in other target organs are affected by the postmenopausal change in hormones. Physiological changes created by the decreased estrogen also impact the skeletal, urogenital, integumentary, neurological, and, cardiovascular systems (Krouse, 1994; Porterfield, 1997; Speroff, Glass, \& Kase, 1994; Utian, 1990).

A study by Burger et al. (1995) investigated hormone changes in 380 women with a mean age of 49.4 years (range: 45.6-56.9). Participants were divided into five groups based upon their reporting of menstrual cycling : (a) regular cycles (Group 1-27\%), (b) change in menstrual flow without a change in frequency (Group 2-23\%), (c) change in frequency without a change in flow (Group 3-9\%), (d) changes in both frequency and flow of menses (Group 4-28\%), and (e) at least three months post last menstrual period (Group 
5-13\%). Data demonstrated significant increases in serum FSH across groups. Compared to Group 1, FSH levels in Group 4 were 53\% higher $(\mathrm{p}=0.0005)$ and Group 5 were 253\% higher (0.0001). Significant decreases in estradiol were also noted between Group 1 and Groups 4 and 5 ( $\mathrm{p}=0.005$ and 0.0001 , respectively). Additionally, women in Group 5 who had no menstrual cycle during the subsequent year demonstrated significantly $(\mathrm{p}=0.05)$ higher FSH and lower estradiol levels than did women in that group who experienced at least one further menstrual cycle during that year. The researchers concluded that these major hormonal changes were associated cross-sectionally with menopausal transition and, subsequently, menopause.

\section{Physiological Impact of Reproductive Hormone Deficiency}

Most symptomatology which occurs during menopause has been directly related to a decrease in estrogen. The negative impact that this decrease has on the physiological function of the skeletal, urogenital, integumentary, neurological, and cardiovascular systems have been investigated by numerous researchers (Rebar, 1994).

Beyond age 40, bone reabsorption begins to exceed the formation of new bone, accelerating after menopause and in some cases leading to bone fractures from osteoporosis. Postmenopausal women within 10 years of the last menstrual period lose bone mineral density (BMD) more rapidly than what can be justified only by the aging process (Speroff, Glass, and Kase, 1994). The Postmenopausal Estrogen/Progestin Interventions (PEPI) trial documented that osteoporosis in the hip and spine was prevalent in approximately $20 \%$ of postmenopausal Caucasian women after age 50 (Trial, 1996). Researchers at the National Osteoporosis Foundation concluded that a minimum of $90 \%$ of all hip and spine fractures among elderly white women should be attributed to the disease. Further study has demonstrated the value of estrogen therapy in preventing bone mineral 
loss which ultimately increases the risk of fractures (Ettinger, 1994; Melton et al., 1997; Richelson, Wahner, Melton, \& Riggs, 1984).

Often primary symptoms prompting women to seek medical intervention are complaints of flushing and diaphoresis which frequently occur at night and often lead to insomnia. Kronenberg (1990), following a review of literature on hot flashes in menopausal women, reported the prevalence of this symptom was highest in the first two years postmenopause and decreased in intensity and frequency in subsequent years. Her research further documented thermoregulatory and cardiovascular changes during a hot flash and suggested that estrogen and progesterone play a role in these changes.

Genitourinary atrophy in menopause causes many uncomfortable symptoms for women including urethritis with dysuria, urgency incontinence, vaginal drying and pruritus, and dyspareunia (Rebar, 1994). Rebar describes the rationale for these symptoms as related to the anatomic changes in genitourinary tract: decreasing size of uterus and adnexa; flattening and shrinking of the labia majora and decreasing size of clitoris; a smaller introitus resulting from loss of subcutaneous fat and elastic tissue in the vulva; and decreasing secretion by the Bartholin glands, shortening and narrowing of vagina, and thinning of the vaginal mucosa which contribute to vaginal dryness, pruritus, and, in some cases, dyspareunia; finally, an increase in the occurrence of vaginal trauma, irritation, and infection occur with changing glycogen content and acidity of secretions from the vaginal epithelium (p. 450).

Researchers Stenberg, Heimer, and Ulmsten (1995) noted that studies investigating the prevalence of urogenital symptoms in postmenopausal women have been rare. While attributing urogenital symptoms to estrogen deficiency, they concluded that these symptoms have been difficult to investigate due to the complexity of the problems. However, because symptoms were relieved by the administration of estrogen therapy, they concurred that a hormonal deficit was implicated as the basis for the symptoms. 
Psychophysiologic complaints, such as fatigue, nervousness, headaches, depression, irritability, and dizziness, may be reported but vary across populations. Researchers also vary as to their belief that these complaints are estrogen-related phenomena (Speroff et al., 1994).

In recent years new research has suggested a possible link between estrogen deficiency and Alzheimer's disease in women. Paganini-Hill and Henderson (1994) conducted a secondary analysis using a case-control methodology with the Leisure World Cohort, a prospective, longitudinal study with 8,877 female residents of a retirement community in southern California. From a cohort of 2,529 females who died between 1981 and 1992, 138 women had been diagnosed with Alzheimer's or other dementia illnesses likely to represent Alzheimer's disease. Researchers determined that the risk of the disease was less in estrogen users when compared to nonusers (odds ratio $=0.69,95 \%$ confidence interval 0.46-1.03). The researchers concluded that the increased incidence of Alzheimer's disease in older women may be due to estrogen deficiency.

Findings of the Baltimore Longitudinal Study of Aging reported by Kawas et al. (1997) added additional support to research findings documenting a protective influence of estrogen on reducing the risk of Alzheimer's disease. This prospective multidisciplinary study conducted by the National Institute on Aging followed 472 peri and postmenopausal women up to 16 years. After adjusting for education, estrogen replacement therapy (ERT) users were found to have a lower relative risk than non-ERT users for developing Alzheimer's disease $(0.46,95 \%$ confidence interval, 0.209-0.997).

Finally, a recent study also indicated that estrogen may have a role in prevention of colon cancer. Researchers in the Cancer Prevention Study II, a prospective mortality study of approximately 1.2 million Americans begun in 1982, examined a cohort of 422,373 postmenopausal women who were cancer-free at the onset of the study. Reported by Calle, Miracle-McMahill, Thun, and Heath (1995), in the 897 colon cancer deaths which were 
documented during the study period, the use of estrogen replacement therapy was associated with a significantly decreased risk of fatal colon cancer.

\section{$\underline{\text { Cardiovascular Risk Factors }}$}

According to statistics published by the American Heart Association (AHA, 1997), myocardial infarction (MI) is the single largest cause of mortality in American women. In 1993 in the U. S., 500,387 women died from cardiovascular disease compared to 457,211 men. Citing various studies, the AHA has alerted women of their increased risk of MI after menopause and of the cardioprotective effect of estrogen and progesterone. The AHA has identified the following risk factors for coronary heart disease:

1. Non-modifiable risk factors:

a. Heredity: African-Americans or individuals with a family history of a first degree relative with cardiovascular disease have greater risk.

b. Male gender.

c. Increasing age: Four out of five individuals dying from myocardial infarction are over 65 years of age. Women who have an MI at these ages are twice as often as men to die within a few weeks of the attack.

2. Modifiable risk factors:

a. Cigarette smoking: The risk of MI is more than twice in a smoker in comparison to a nonsmoker, and smokers are more likely to experience a fatal MI. If combined with a family history of heart disease, smoking greatly increases the risk. Women who quit smoking reduce their risk after 2-3 years cessation to a risk comparative to that of a nonsmoker.

b. High blood cholesterol levels. 
c. Hypertension.

d. Physical activity: Participation in even modest levels of low-intensity activity is beneficial if done regularly and long term.

3. Other contributing factors

a. Diabetes Mellitus: According to AHA statistics, greater than $80 \%$ of individuals with diabetes die of some form of cardiovascular disease.

b. Obesity: Although not an independent risk factor for cardiovascular disease, obesity is associated with other risk factors such as hypertension, dyslipidemia, and diabetes.

c. Stress: Studies have suggested that a relationship exists between cardiovascular disease and an individual's life stress and socioeconomic status. This factor may contribute to other risk factors such as smoking, obesity, and hypertension.

\section{$\underline{\text { Impact of Reproductive Hormone Deficiency on Cardiovascular Disease }}$}

The cardiovascular system is significantly impacted by menopausal estrogen deficiency (Gupta \& Rymer, 1996). Cardiovascular disease primarily results from atherosclerosis in major vessels. Research has shown that prior to menopause higher highdensity lipoprotein (HDL) levels and lower cholesterol levels are present. After menopause, however, atherogenic lipids increase, with an associated increase in atherosclerotic effects in arteries and increased risk for cardiovascular disease (Barrett-Connor, 1995; Gupta \& Rymer, 1996; Rebar, 1994). Reported by Nabulsi et al. (1993), the Atherosclerosis Risk in Communities Study followed 4,958 postmenopausal women, classifying them into four groups: (a) current users of unopposed estrogen replacement, (b) current users of estrogen and progesterone replacement, (c) current nonusers who had previously been on some type 
of hormone replacement, and (d) current nonusers with no past history of hormone replacement use. Results demonstrated that current users, in comparison to both classifications of nonusers, had higher mean levels of HDL and lower mean levels of LDL but higher triglyceride levels. Lower fasting serum glucose levels were also documented.

Loss of estrogen affects vessel endothelium and vasodilating and antiplatelet aggregation factors which then predispose the postmenopausal woman to negative cardiovascular outcomes (Rosselli, Imthurn, Keller, Jackson, \& Dubey, 1995). In an animal study, Wellman, Bonev, Nelson, and Brayden (1996), examined gender differences in coronary arteries from rats. Results demonstrated pressure-induced arterial vasoconstriction was greater in estrogen-deficient male or ovariectomized rats when compared with similar arteries of female ovariectomized rats receiving physiological levels of estrogen replacement. Researchers concluded that physiological levels of estradiol increased the diameter of pressurized coronary arteries and could be a reason for the cardioprotective effect of estrogen in women.

Further studies in peri and postmenopausal women have suggested that estrogen is active both in vascular smooth muscle and endothelium, and its effect on hemodynamic characteristics can be mediated by the vasoregulatory hormone, nitric oxide (Ramsay, Johnson, Leone, \& Steer, 1995; Rozenberg, Liebens, Vandromme, Hotimsky, \& Rijsselberge, 1994; Sudhir, Jennings, Funder, \& Komesaroff, 1996). Nitric oxide is a substance secreted by vessel endothelium, a monolayer of cells lining the intimal surface of the circulatory system which aids in the moderation of coronary tone (Collins \& Beale, 1996). Studies have found that oxidized lipids inhibit nitric oxide (NO), whereas estrogen is a potent antioxidant of lipids. The cardioprotective effect of estrogen, therefore, may be related to enhanced NO production which results in relaxation of the vessel and slows the development of atheroma. 
Hormone Replacement Therapy

Despite increasing amount of scientific data published about its benefits and risks, many questions about HRT remain unanswered, creating a degree of uncertainty in the general population. Most studies until recently have been observational, retrospective, or correlational. Results from the Postmenopausal Estrogen/Progestin Interventions (PEPI), a prospective, 3-year clinical trial, corroborated previous studies documenting the cardioprotective effect of HRT through its reduction of lipids (Trial, 1996).

\section{History}

Since the 1930s, oral estrogen has been prescribed to treat the symptoms of menopause. In the 1970s, estrogen was one of the top five prescriptions sold in the United States. However in the mid 1970s, several epidemiological studies reported a relationship between estrogen use and endometrial hyperplasia and cancer with 2,900 deaths from the disease reported in 1984 . Use of estrogen therapy decreased by $40 \%$. Following a reexamination of the side effects, hormone replacement therapy was introduced in the 1980s with a progesterone added to the former unopposed estrogen to prevent endometrial hyperplasia. This alteration was accepted by healthcare providers and recommended by the American College of Obstetrics and Gynecology (Voda, 1993).

\section{Pharmacology}

Hormone replacement therapy can be administered through a variety of pharmacological preparations, non-oral and oral. Non-oral routes of administration such as transdermal patches, transvaginal gels and rings, subcutaneous pellets and intranasal sprays, and non-traditional alternatives for estrogen such as the phytoestrogens in soy products currently exist. The most common and most frequently prescribed preparation is 
estrogen extracted from the urine of pregnant mares. Complex pharmacokinetics of conjugated equine estrogen (CEE) are a result of various estrogenic components of the drug. All oral preparations are converted by the body to estrone sulfate and rapidly metabolized in the liver (Scharbo-Dehaan, 1996).

Current clinical practice guidelines dictate that postmenopausal women with intact uteri be prescribed HRT consisting of estrogen with the addition of a progestin to prevent endometrial hyperplasia (American College of Physicians, 1992; Barrett-Connor, 1995). Research has provided conflicting results regarding the physiological effect of progesterone. Grady et al. (1992) and Notelovitz (1989) reported on studies indicating that progesterone may neutralize the positive effect of estrogen. However, in a randomized, crossover study by Koh et al (1997), no diminished effect on fibrinolysis or lipoprotein in postmenopausal women was observed. Additionally, research published by BarrettConnor, Wingard, and Criqui (1989) showed no significant differences in lipid levels for users of estrogen only, compared to users of both estrogen and progesterone.

The American College of Physicians (1992) recommended one of two drug administration regimens: continuous or cyclic. With a continuous regimen, the usual prescribed dose is estrogen $0.625 \mathrm{mg}$ and medroxyprogesterone acetate (MPA) $2.5 \mathrm{mg}$. The cyclic regimen consists of daily estrogen $(0.625 \mathrm{mg})$ and adding a progestin, usually MPA 5-10 mg, for the last 12 to 14 days of the month.

No single protocol exists for the administration of HRT, nor one ideal regimen that satisfies all women. The ideal dose is one which provides the lowest dose of estrogen and effectively treats reported symptoms of menopause as well as reduces the potential for morbidity/mortality which has been linked to estrogen deficit. 
$\underline{\text { HRT Use and Compliance }}$

Voda (1993) reported that fewer than $20 \%$ of postmenopausal women in the United States use HRT. Additionally, women who chose to initiate therapy remained on the drugs an average length of only 9 months. Utian and Schiff (1994) reported results of a survey sponsored by the North America Menopause Society (NAMS) and conducted by The Gallup Organization to: (a) explore women's knowledge about menopause; (b) identify obstacles which occur in the patient/physician communication regarding menopause; and, (c) identify issues of concern to women over age 45 . Using a proportionate, stratified random digit telephone sample drawn from telephone exchanges serving the continental U.S., 833 interviews were conducted with women between the ages of $45-60$ between May and June, 1993. Of the total sample, 53.5\% were premenopausal and $43.2 \%$ were postmenopausal. Only 27 (3.3\%) were listed as unknown status. Three hundred and twelve women (37.5\%) had previously undergone hysterectomy. Caucasians comprised $86.4 \%$ and blacks, $10.2 \%$ of the total sample. The majority of the women $(63 \%)$ had no college education. When questioned about HRT, approximately three in five (58\%) of the women responded that they had never taken HRT. Only $34 \%$ were currently taking hormones and $8 \%$ reported previous use of HRT. Women who had received a hysterectomy were more likely to currently use HRT (53\% compared to $21 \%$ ). The rationale most often given (34\%) by previous users for discontinuing use of HRT was due to drug side effects (Utian \& Schiff, 1994).

Faulkner, Hutchins, and McCollam (1997) conducted a retrospective study of HRT compliance from a large prescription claims database in Arizona. The study followed for one year, 79,956 women, ages 40-59, who had been newly prescribed HRT. Preliminary results demonstrated that only $46 \%$ of the patients were compliant with HRT therapy at the end of one year. 


\section{Benefits of HRT}

Research examining the pharmacological benefits of HRT to counter the effects of cardiovascular disease, osteoporosis, urogenital changes, vasomotor flushing, cognition, and colon cancer have been fully discussed under the earlier section, reproductive hormone deficiency. A short synopsis of those benefits is reviewed in this segment.

In a manuscript reviewing the literature on hormone replacement therapy, Rebar (1994) reported on multiple studies which have demonstrated the benefits of HRT.

Research has shown that HRT has a cardioprotective function by regulating plasma lipoprotein levels causing a decrease in LDL and increase in HDL. Improved blood flow to tissue, including the myocardium, increased coronary vasodilatation through mediation of the endothelial-derived relaxing factor of nitric oxide, and modulation of local vascular wall fibrinolytic activity which reduces the development of atherogenesis, also have important implications from the use of HRT for the prevention and treatment of cardiovascular disease.

Rebar also reported on studies which found that use of HRT prevented and treated osteoporosis in postmenopausal women. Researchers at the National Osteoporosis Foundation concluded that use of HRT ultimately reduced the risk of fractures by preventing bone mineral loss which occured in postmenopausal, estrogen-deficient women (Melton et al., 1997).

Symptoms caused by postmenopausal changes in the genitourinary tract have also been eliminated or decreased following use of HRT. Urethritis with dysuria, urgency incontinence, vaginal drying, pruritus, and dyspareunia resulting from postmenopausal anatomic changes have been relieved by treating the estrogen deficiency. Additionally, vasomotor complaints of flushing, hot flashes, and diaphoresis have been eliminated by administration of exogenous estrogen (Rebar, 1994). 
Although acknowledging that research investigating the effect of HRT on mood and psychological function have been few and poorly controlled, Rebar suggested that HRT has a positive effect on cognition, emotions, and behavior. A case-control study within the Leisure World Cohort by Paganini-Hill and Henderson (1994), and research by Kawas et al. (1997), suggested that HRT may be useful for preventing or delaying the onset of dementia caused by Alzheimer's disease. A recent study by Calle et al. (1995) also has shown a link between HRT and a reduced risk of colon cancer.

\section{$\underline{\text { HRT and Breast Cancer }}$}

Evidence suggesting that estrogen use increases the risk of breast cancer is a continuing source of concern for postmenopausal women, although conflicting data exist on the actuality of this risk. A meta-analysis of studies investigating a link between postmenopausal estrogen therapy and risk of breast cancer found no overall increase with short term hormone use (Steinberg, Thacker, \& Smith, 1995). In contrast, two other metaanalysis of studies on long-term estrogen use found that breast cancer risk increased with duration of estrogen use, with a $25-30 \%$ increase after ten years or longer of sustained therapy (Grady \& Ernster, 1991; Steinberg, Smith, Thacker, \& Strough, 1994). Ettinger, Quesenberry, Schroeder, and Friedman (1997) also found a link between breast cancer and long-term estrogen use. From a longitudinal study of 454 women who were followed an average of 31.1 years after menopause, 232 women had taken estrogen for 17.2 of those years. At the conclusion of the study, $72 \%$ of the estrogen users and $57 \%$ of nonusers were still participating in the study. The researchers found a twofold risk of breast cancer with long-term use of estrogen. 
Heart Rate Variability

Heart rate variability is defined as the cyclic change or fluctuation of heart periods (R-R intervals) around the mean heart rate over time. The amount of short and long term variability in heart rate provides a measurement of autonomic nervous system (ANS) balance, delineating between sympathetic and parasympathetic components of autonomic control . The non-invasive clinical method for assessing measurement of HRV is by 24 hour Holter monitoring which provides a continuous recording of electrocardiographic data. Analysis of the data occurs across two domains, frequency and time. Frequency domain data are mediated by the sympathetic and parasympathetic nervous system control and are reported by the values of Total Hertz, High Hertz, Low Hertz, and Ratio of Low to High Hertz. Time domain data are a general measure of autonomic nervous system balance and reported by the values of pNN50, rMSSD, SD, SDNN, and SDANN (See Table 1) (Cowan, 1995; Keehn, 1992).

\section{Clinical Applications}

Measurements of 24-hour heart rate variability (HRV) have been shown to be good predictors of physiological stress and mortality, especially for cardiovascular disease. Sympathovagal imbalance in coronary artery disease and hypertension may be detectable, and low heart rate variability has been associated with an increased risk for sudden cardiac death in patients with diabetes and in those who have experienced a myocardial infarction.

Kleiger, Miller, Bigger, and Moss (1987) were among the early researchers to report on clinical applications of HRV. They investigated HRV as a predictor of long term survival following an acute myocardial infarction (MI). In a multi-center study of patients from nine hospitals located in five U.S. cities, 808 patients younger than 70 years old who had survived the acute phase of MI were enrolled in the study. One HRV variable, 
identified as the 24-hr SD by Kleiger, was defined as the standard deviation of RR intervals around the average This value is now labeled SDNN (Keehn, 1992). Participants were divided into three groups by their SDNN value: (a) < 50 ( $\mathrm{n}=125)$, (b) 50-100 ( $\mathrm{n}=472)$, and (c) $>100(n=211)$. Results indicated that participants whose SDNN values were less than $50 \mathrm{~ms}$ had approximately a four-fold increased risk of dying during follow-up when compared with participants whose SDNN value was more than $100 \mathrm{~ms}$.

Saul et al. (1988) conducted a study to evaluate the differences in cardiac autonomic balance in 21 normal control participants and 25 participants with chronic stable congestive heart failure (CHF). HRV results corroborated other studies demonstrating a diminished vagal response and a preserved sympathetic modulation of heart rate in participants with CHF. Additionally, these results were not influenced by age as was noted in HRV values in normal controls.

Decreased HRV, specifically in values measuring parasympathetic modulation, has been documented in individuals following sudden cardiac arrest (Cowan, 1995; Magid et al., 1987; Martin et al., 1987). Cardiac arrest is caused by electrical instability, primarily augmented by sympathetic hyperactivity. Following an arrest, it has been hypothesized that loss of parasympathetic tone allows for this uncontrolled sympathetic hyperactivity increasing the risk for event mortality. These studies indicate that analysis of HRV may identify patients at higher risk for sudden cardiac death.

The association of decreased HRV with an increased risk for new cardiac events was studied in a sample of 2501 participants, mean age of 53 years with no clinically apparent signs of cardiovascular disease, in the Framingham heart study. During a mean follow-up of 3.5 years, 58 individuals experienced a cardiac event. Adjustment for age, sex, cigarette smoking, diabetes, left ventricular hypertrophy, and other relevant risk factors was made in the analysis of data. Results showed all HRV measurements with the exception of the Low/High ratio were significantly associated with risk for a cardiac event 
( $\mathrm{p}=0.0016)$. Findings suggested that HRV analysis could offer healthcare providers valuable prognostic data on cardiovascular disease (Tsuji et al., 1996a).

Recent research has also indicated that HRV analysis may be used to further define psychologically based illnesses such as depression and anxiety by evaluating the sympathovagal balance. Tiller, McCraty, and Atkinson (1996) conducted a study with 20 individuals trained in the Freeze-Frame bio-feedback type technique where they were taught to recognize stressful feelings and initiate interventions to regain emotional/physical balance. Participants were monitored for 24 hours during a normal business day in their workplace and instructed to use the Freeze-Frame technique on at least three occasions when they were sensing stress or feeling out of balance. During positive emotional states, a marked increase in values representing parasympathetic mediation was noted.

\section{$\underline{\text { Influences on HRV }}$}

Although studies have indicated that a variety of factors may influence HRV values, some results have been conflicting.

Age. Increasing age has been implicated for decreasing HRV (Liao et al., 1995; Murata, Landigran, \& Araki, 1992; Schwartz, Gibb, \& Tran, 1991; Veerman, Imholz, Wieling, Karemaker, \& Montfrans, 1994). However, Reardon and Malik (1996), although identifying a significant difference $(\mathrm{p}=<0.05)$ in the HRV triangular index which gives an estimate of overall HRV measurement, reported no significant change in vagal measurements of rMSSD with age. They evaluated HRV on 56 healthy participants between the ages of 40 and 102 years, 39 of whom were women. None had a history of heart disease or were taking medication which could affect cardiac autonomic function. Parameters were compared between participants younger and older than 70 years. The researchers concluded that the time domain short term component (rMSSD) reflecting vagal 
modulations of heart rate was not affected by age. Saul et al. (1988) has also reported that the effect of age on HRV is not a significant factor in patients with congestive heart failure.

Gender. In a study to describe the effects of gender and age on HRV in healthy participants $(\mathrm{N}=111)$, Cowan et al. (1994) examined 40 men (mean age $=57 \pm 15)$ and 71 women (mean age $=52 \pm 15$ ). Results showed significantly lower values in healthy women than in healthy men for all variables except the high frequency (High Hertz) and the two time domains representing vagal modulation (rMSSD and pNN50). Another study by Pace et al. (1997) examined gender differences in 253 participants (123 with diabetes) awaiting renal/renal-pancreas transplantation. Researchers found lower mean HRV scores for women although no significant difference between gender at an alpha of 0.05 was noted.

Pharmacological Intervention. Drug therapy may influence HRV and should be considered when analyzing HRV results. Van Ravenswaaij-Arts et al. (1993) reported that spectral analysis helped to quantify sympathetic and parasympathetic activities of drugs such as beta-blockers and calcium channel blockers. In normotensive adults, fast fluctuations in heart rate were mediated through the vagal influences of atenolol. The researchers further reported on a study by Guzzetti and colleagues which investigated the effect of atenolol in hypertensive patients. An increase in the parasympathetic high frequency value (High Hertz) was observed corresponding to a decrease in the sympathetic low frequency value (Low Hertz). Additionally, van Ravenswaaij-Arts found that calcium channel blockers had varying responses on HRV. In postinfarction patients, Diltiazem reduced low frequency HRV similar to those values demonstrated by the beta blockers. Nifedipine, conversely, did not reduce sympathetic activity. Sedatives, analgesics, and anesthetics were also found to negatively influence HRV.

Salo et al. (1996) investigated the effect of drugs on HRV. Thirteen hypertensive diabetic participants, mean age 48 ( \pm 4 years), were assessed during treatment with a cardioselective beta blocker (metoprolol) and an ACE inhibitor (enalapril). Both drugs 
decreased blood pressure comparably in the sample. Metoprolol also decreased heart rate and HRV low frequency values. Enalapril did not significantly alter HRV. Noting that HRV was significantly abolished in participants with hypertension, diabetes, and autonomic neuropathy, the authors suggested that an ACE inhibitor may be a better alternative for individuals with related disorders.

Physical Activity. Positive effects of exercise on HRV have been demonstrated in various studies conducted with older patients (Levy et al., 1992) and those with chronic illness such as diabetes (Howorka et al., 1997) and coronary artery disease (Osterhues, Hanzel, Kochs, \& Hombach, 1997). In postmenopausal women, a study by Davy, Miniclier, Taylor, Stevenson, and Seals (1996) examined HRV in 9 physically active women (mean age $=53 \pm 1$ year) and 11 age-matched controls (mean age $56 \pm 2$ years). The time domain variables were significantly higher in the active women $(\mathrm{p}=<0.05)$. Mean frequency values were also increased compared to those of the less active women.

Substance Abuse. Chronic alcoholism has been associated with autonomic neuropathy resulting from impairment in vagal modulation of cardiac function (Omboni, Parati, Rienzo, Wieling, \& Mancia, 1996). Smoking has also been associated with lower values of HRV (Tsuji et al., 1996b).

\section{$\underline{\text { HRV and Estrogen }}$}

According to Collins and Beale (1996), estrogen has beneficial effects on the autonomic nervous system by modulating the influence of sympathetic/parasympathetic influences. A study by Huikuri et al. (1996) used HRV to examine sex-related differences in autonomic modulation of heart rate in randomly selected, age-matched men and women. A sample of 186 women and 188 men, mean age $50( \pm 6)$ years demonstrated gender differences in cardiovascular autonomic regulation suggesting that the vagal responses to cardiovascular unloading was attenuated in women. A secondary analysis of data was 
reviewed on 92 women, separated into two groups based upon estrogen use; each group consisted of 46 women (mean age $50 \pm 4$ years). The researchers found that heart rate variability was higher for postmenopausal women on estrogen replacement therapy (ERT) compared to women without ERT and suggested that hormonal factors may influence changes in autonomic modulation. Significant differences were noted between the two groups of women in time domain variables of $\mathrm{SD}(\mathrm{p}=0.01)$ and $\mathrm{pNN} 50$, a measurement of vagal function $(\mathrm{p}=0.05)$. Women on estrogen also demonstrated significantly better values for the frequency domain variables (High Hertz: $\mathrm{p}=0.05$; Low Hertz: $\mathrm{p}=0.01$ ).

A second study by Rosano et al. (1997) supported previous results that postmenopausal women have an increased sympathetic tone which may cause an abnormal control of the cardiovascular system. A sample of 30 women, mean age $56( \pm 6)$ years, nonsmokers, with no symptoms or evidence of cardiac disease underwent 24-hour ambulatory ECG monitoring. Baseline HRV results demonstrated an imbalance in autonomic control with primarily sympathetic hyperactivity demonstrated by low values of high frequency (High Hertz) and a high low/high frequency ratio. Eighteen women were subsequently placed on transdermal ERT and 12 women refused ERT, but served as controls for the study. HRV analysis was repeated at four weeks and four months. Significant changes $(\mathrm{p}=0.05)$ were noted at four weeks in the ratios reflecting parasympathetic modulation (High Hertz, rMSSD) and sympathovagal balance observed by a decrease in the Low/High Ratio. All values were significantly improved at the four month retest with the high and low frequency values and $\mathrm{pNN} 50$ significant at $\mathrm{p}=0.01$ suggesting that an "extra period of estrogen exposure is needed to stabilize the autonomic control of the cardiovascular system in symptomatic menopausal women" (p. 816). 
$\underline{\text { Related Research Studies }}$

A current research study by Engelhardt, Pace, Hathaway and Andersen (1997) is examining the relationship of female sex steroids to autonomic function (AF). In seeking to evaluate the hypothesis that the autonomic nervous system is a significant mediator of the protective effects of estradiol on the cardiovascular system, the researchers are conducting vasomotor and cardiac evoked testing, in addition to 24-hour heart rate variability, within four groups of women based upon reproductive status. Preliminary results suggest that PMW on HRT have more optimum AF levels than PMW not on HRT in some, but not all, tests. More optimum levels were observed in the HRV time domain tests of SD, SDNN, and SDANN. In this study, age effects on HRV have been evident.

A secondary data analysis by Pace, Engelhardt, \& Hathaway (1996) examined gender differences in autonomic function within a population of 253 patients awaiting kidney/kidney-pancreas transplantation. Results demonstrated that women demonstrated poorer values which were significant/approaching significance in 6 of 16 AF variables tested when compared to men with similar pathology. Lower, although not significant, AF values were found by Cashion (1998) in healthy female controls when compared to healthy male controls. Both studies suggest that estrogen may influence autonomic nervous system function.

\section{Summary}

A significant amount of literature, as previously reviewed, is available about menopause addressing the effect of decreased reproductive hormones on biological processes. Metabolic alterations occurring as a result of this deficiency impact multiple body systems, especially the cardiovascular system. Many studies have also addressed the effects of hormone replacement therapy in preventing morbidity and mortality resulting from these metabolic alterations. Limited research, however, has been conducted 
investigating the effect of menopause, or the effect of postmenopausal HRT, on heart rate variability. This study, therefore, was conducted to provide additional descriptive data on HRV in postmenopausal women and to investigate differences in HRV across selected cardiovascular risk factors before and after the administration of HRT. 


\section{CHAPTER III}

\section{METHODOLOGY}

Research has demonstrated that increased risk of cardiovascular disease (CVD) exists in postmenopausal women, and hormone replacement therapy decreases cardiac risk. Because analysis of HRV has been used to predict cardiac morbidity and mortality, consequently this study was designed to investigate cardiovascular health of PMW using HRV. Selected factors identified by the American Heart Association as increasing risk of CVD were also investigated in their relation to HRV in PMW. This chapter will discuss research aims, study design, sample and site, drug intervention protocol, instrumentation, and procedures for a study investigating the effect of postmenopausal HRT on HRV.

\section{Research Aims}

Three specific aims were identified for conducting this study:

1. Describe heart rate variability in postmenopausal women, ages 45-60.

2. Describe the differences in heart rate variability in postmenopausal women before and after six weeks of hormone replacement therapy.

3. Describe heart rate variability in postmenopausal women relative to selected cardiovascular risk factors before and after six weeks of HRT.

\section{Research Design}

This research study was conducted in the Mid-South at two community-based ambulatory care clinics and a suburban church using a therapeutic intervention, one-group 
pre-test/post-test design. An early decision was made to place minimal limitations on the exclusion criteria for participation. Because many women in community settings, although healthy, have variations in their lifestyle adaptation, exposure to environmental factors, and presence of concomitant disease, the study was designed to allow women usually seen in this population to serve as their own controls. According to Campbell and Stanley (1963), this design effectively controls for internal validity issues. Historical events such as seasonal and time changes, variations in physiological measurements such as temperature, pulse, respirations, blood pressure and weight, and environmental factors such as stress, change in diet and exercise which might influence HRV values were assumed to produce similar effects in both the pre-test and repeated measures post-test values of the same individual over a six-week time period.

\section{Sample and Site}

This study used a non-probability, purposive sampling to obtain representative proportions of participants with varying ethnic and socio-economic demographic characteristics. Following approval to conduct the research from the Institutional Review Board at the University of Tennessee, Memphis and from the Medical Director for the community-based clinics (see Appendix A), participants were recruited from the following sites: (a) a community-based urban family practice clinic in the eastern geographic area of the city, (b) a community-based inner city family practice clinic, and (c) a suburban church. Recruitment in these settings allowed for a diverse population of participants with varying ethnic and socio-economic demographic characteristics.

The following inclusion/exclusion criteria were established for the study:

Inclusion Criteria: $\quad$ 1. Women between the ages of 45 and 60 years;

2. Self-reported amenorrhea for 12 months; 
3. Hormone levels: FSH $\geq 30 \mathrm{mic} / \mathrm{ml}$ and Estradiol $\leq 30 \mathrm{pg} / \mathrm{ml}$;

4. Expressed willingness and ability to take an oral medication;

5. Expressed willingness to complete the six week study.

Exclusion Criteria: 1. Past medical history of hysterectomy;

2. Undiagnosed abnormal vaginal bleeding;

3. Past medical history of breast cancer;

4. Family history of first-line relative with breast cancer;

5. Current use of hormone replacement therapy. However, individuals on current HRT could participate following a 6-week wash-out period. Two women in the study met this criteria.

Although not considered as inclusion/exclusion criteria, participants were encouraged to notify their primary care provider of their participation in the study. Additionally, because of recognized health maintenance benefits, participants were also encouraged to have their yearly gynecological exam and mammogram if it had been one year or longer since their last evaluation.

Twenty-nine women met study criteria with three declining to participate. Twentysix women who met the inclusion criteria and agreed to participate in the study were enrolled. Twenty-four women completed the study. One of the enrolled women elected to discontinue HRT after three weeks, secondary to medication side effects; a second woman had no phone and was lost to follow-up.

\section{Drug Intervention Protocol}

The intervention protocol for this study was based upon the 1992 "Guidelines for Counseling Postmenopausal Women about Preventive Hormone Therapy" published by the American College of Physicians (1992). The HRT guidelines for women who have intact 
uteri is the administration of estrogen with the addition of a progestin. The frequently prescribed clinical regimen of oral conjugated estrogen, $0.625 \mathrm{mg}$ and medroxyprogesterone acetate, $2.5 \mathrm{mg}$ taken on a daily basis, was used as the drug intervention for this study. Drug samples for distribution to patients through provider offices were supplied by a pharmaceutical representative of the company. The drugs were combined in a single peach tablet, packaged individually on a blister card marketed as PremPro $^{\mathrm{TM}}$, a trademark of Wyeth-Ayerst Laboratories. A package insert (Wyeth-Ayerst Laboratories, 1997, January) and a consumer booklet (Wyeth-Ayerst Laboratories, 1997, April) supplied by the company were distributed to each participant at the onset of the study. Potential side effects were discussed with each individual The medication samples, a 44-day supply of tablets, were dispensed by the principal investigator, a certified Family Nurse Practitioner with prescriptive authority from the Tennessee State Board of Nursing (see Appendix B).

Instrumentation

\section{Demographic}

Two demographic/health history forms (see Appendix C) designed by the principal investigator were used during the study. The General Information data form was completed by the participant and documented demographic information including age, race, marital status, education, and social behaviors including smoking, exercise, and alcohol consumption. Information was also solicited on reproductive history, current and past medical history, and family history. The Pre/Post-Test Health Form was completed by the primary investigator at the time of enrollment and retesting. The form verified basic demographic information, validated adherence to the inclusion/exclusion criteria, and 
allowed for documentation of physiological variables such as pulse, blood pressure, height, and weight. In addition, the date for retesting was noted.

\section{$\underline{\text { Hormone Assays }}$}

Analysis of serum estradiol and follicle stimulating hormone (FSH) values were performed by automated chemiluminescence immunoassay using the CIBA-Corning ACS: 180 Plus System ${ }^{\circledR}$ manufactured by Chiron/Diagnostic Corporation. Intra-assay coefficients of variation were noted as $6.49 \%$ for estradiol and $3.56 \%$ for FSH. Inter-assay coefficients of variation were noted as $11.1 \%$ for estradiol and $4.98 \%$ for FSH (Chiron/Diagnostic, 1996). Automated hormonal assays were performed by licensed medical laboratory technicians at a certified laboratory directed by the University of Tennessee College of Medicine, Department of Obstetrics and Gynecology.

\section{Heart Rate Variability}

Heart Rate Variability (HRV) was measured by use of the Marquette Electronics ${ }^{\circledR}$ Ambulatory ECG Analysis and Editing Systems, version 5.8 software program with Series 8500 Holter recording monitor (Marquette Electronics, 1990). The Holter monitoring equipment provides continuous recording of 24-hour non-invasive electrocardiographic data. Marquette ${ }^{\circledR}$ HRV software analyzes beat to beat variability of heart waveforms, providing $\mathrm{R}$ wave detection and offering discrimination and reproducibility.

Standard monitoring procedures provided by the Physiological Function Laboratory and supervised by the director of the laboratory at the University of Tennessee, Memphis, located at the W. F. Bowld Hospital were used during this study. The standardized Holter monitor protocol (see Appendix D) developed by the laboratory research team was used to insure consistency in testing. The PI for this study connected all participants to the Holtor monitor to insure reliability of electrode placement. The PI was instructed in the protocol 
and was observed in a correct return demonstration by the research technician. This technician had received intensive training in the procedure and was currently testing approximately 15 patients a week at the laboratory using the established protocol.

HRV data analysis for this study was conducted only by the PI to insure reliability of analyses. Two recordings of 24-hour electrocardiographic data were obtained on each participant pre-intervention and post-intervention, six weeks later. Ten percent of both the pre- and post-intervention tapes were reanalyzed by the PI to insure intra-rater reliability.

The analysis of HRV was done by first downloading each tape into the Marquette ${ }^{\circledR}$ software program which provided a mechanical analysis of beat morphology. The QRS beats were divided into multiple classifications by the computer program but were manually reviewed by the PI and reclassified, when necessary, for correct labeling of normal, artifact, ventricular ectopy, and unclassified beats. Incorrect labeling was edited by the PI because undetected or misclassified beats could add artificial variability, reducing internal validity (Keehn, 1992).

Following manual validation of beat classification, HRV analysis was performed on each tape, calculated by the Marquette ${ }^{\circledR}$ software (Marquette Electronics, 1990) using a Fast Fourier Transform algorithm with the resulting expression transformed into Hertz frequency values by an additional computerized mathematical calculation. Non-spectral measurement of time domain components of HRV were also calculated by the Marquette ${ }^{\circledR}$ software. Calculations of HRV values have been reported reproducible across intervals ranging from 3 to 65 days in 14 normal participants (Kleiger et al., 1987). Quality assurance data provided by the manufacturer stated that "calculations and statistics of the Marquette HRV program can be verified from laboratory measurements of predictable test signals" (Keehn, 1992) p. 139.

Cowan (1995) reported validity of the time domain variable measurements showing high correlations $(r=.90)$ among the individual values. Strong correlations of the time 
domain variables with the frequency domain variables were also demonstrated. Cowan also documented high correlations between frequency domain variables using two different methods of HRV analysis, Fast Fourier Transform and Autoregressive ( $r=.97$ and r=.99). Kleiger et al (1991) reported data on reliability attesting to the reproducibility of 24-hour HRV measurements.

Normative data and reliability and validity studies for data obtained at the UTM lab is ongoing. Additionally, a current study by Engelhardt, Pace, Hathaway, and Anderson (1997) is currently being conducted to determine normative values of HRV in healthy women at varying points in their reproductive history.

\section{Procedures}

\section{$\underline{\text { Recruitment/Enrollment }}$}

Letters were sent to the providers at the clinics notifying them of the study, and posters explaining the study were posted at all three sites. Patients who reported to the clinics during the period between September, 1997 and January, 1998 and met the study criteria were invited to participate. Non-clinic participants were recruited by the PI through distribution of flyers at a suburban church. Individuals who were interested in participation were directly contacted by the PI, study details were explained, and a mutually agreed upon date and time scheduled for testing.

Twenty-six individuals who met the inclusion and exclusion criteria were enrolled in the study. The study protocol was explained to each participant by the PI and a signed consent form was obtained (see Appendix E). Appropriate data was obtained on the PreTest Health Form, and the self report Demographic Information Record was distributed to the participant with verbal instructions on how to complete the form (see Appendix C). Participants were encouraged to alert their provider of their participation in the study and to 
continue with routine health maintenance and/or follow-up visits. For any medical questions or concerns, they were instructed to discuss those issues with their provider. For any questions on follow-up related to the study, a phone number providing 24-hour access to the PI was provided.

At the time of enrollment, a return appointment time was made for repeat testing on or between days 40 and 44. Participants were reminded of and confirmation obtained by phone one week prior to the scheduled post-test appointment.

\section{$\underline{\text { Blood Collection for Hormone Assays }}$}

The procedure for venipuncture was explained to the participant, and blood was drawn by the PI, a Family Nurse Practitioner, or a medical assistant trained and certified in venipuncture technique. Following universal precautions, $20 \mathrm{ml}$ of venous blood was obtained in two serum separator blood collection tubes. Tubes were delivered to the onsite, Level Two CLIA certified clinic laboratory by the PI and handled according to established laboratory protocol by a licensed Medical Laboratory Technician. The blood was centrifuged to separate plasma and serum. The serum was placed in a plastic blood container tube and placed in the freezer, again following established laboratory protocol. All specimens were batched and frozen until hormone assays were run by a certified laboratory with the University of Tennessee, Memphis, College of Medicine (see instrumentation). Results were documented on a lab sheet and returned to the PI.

\section{$\underline{\text { Drug Intervention Protocol }}$}

An envelope with 44 individual PremPro ${ }^{\mathrm{TM}}$ tablets in blister packaging was given to each participant, and they were instructed that more tablets were distributed than they might

have to take. All participants were informed of potential side effects of the medication, and a drug insert information sheet and consumer brochure was distributed. To promote 
compliance with taking the daily medication, they were given a Drug Documentation Calendar (see Appendix F) and instructed to mark with an "X" each day that the medication was taken. Should the tablet not be taken, they were instructed to circle the day and not to take any missed pills. The calendar was to be returned at the time of retesting. Additionally, participants were instructed to keep a diary of any changes which might occur during the six-week study time, such as change in prescribed medication, change in exercise level, hospitalization, unusual stressor, and to note the change on the Drug Documentation Calendar.

\section{$\underline{\text { Heart Rate Variability }}$}

Twenty four hour ambulatory Holter monitoring was conducted on each participant at enrollment and again on completion of the study on or between days 40 and 44 . The chest area of the participant was cleaned by vigorous rubbing of a pre-packaged alcohol swab and then by a dry gauze pad. Five chest electrodes were placed in a modified Lead One position (see Appendix D). Lead wires were attached to the monitor according to manufacturer's instructions. Both the electrodes and leads were securely taped to the participant's chest to prevent dislodgment. The electronic recording monitor, weighing approximately 12 pounds, was placed in a purse-like holder with a strap allowing the

participant to wear it around their neck or waist. A one page diary (see Appendix D) supplied by the Physiologic Function Laboratory with the monitors, was given to each participant with instructions to note the time of meals, exercise, any unusual stressors, and sleeping time on the diary. Additionally, the following directions were given to each participant orally by the PI:

1. Monitoring will be continuous for the 24-hour period. Do not disconnect the monitor before the specified time given by the PI. 
2. Do not shower or allow the electrodes to become wet. Should the tape become loose on the electrodes, reapply new tape.

3. At the end of 24 hours, remove the electrodes from the chest. Do not disconnect any leads. Return the equipment, unopened, with the data form and diary, to the site at which testing was initiated.

\section{Summary}

Enrollment with venipuncture, Holter Monitor application, and instructions took approximately 60 minutes. Retesting took approximately 25 minutes because no further enrollment instructions were necessary. Participants who elected to continue HRT on completion of the study were given a prescription for PremPro ${ }^{\mathrm{TM}}$. For individuals who had been recruited at the clinic sites, this procedure was documented in their medical record. For non-clinic participants, a prescription for two months was given by the PI with instructions to contact their primary care provider for follow-up and continued drug prescription refills. They were informed that results would be mailed to them following completion of the study

Except for venipunctures and analysis of hormonal assays, data collection was completed by the PI. HRV analyses and all data entry for statistical computation was also completed by the PI.

\section{Protection of Human Subjects}

Approval to conduct the study was obtained from the Institutional Review Board of the University of Tennessee, Memphis. Additional approval for clinic site utilization was obtained from the executive and medical directors for the clinics (See Appendix A). 
Although a drug intervention was involved with the study, dosage was at recommended therapeutic levels and minimal side effects were expected for the 6-week study protocol. Heart rate variability testing by the Holter monitor was expected to cause no discomfort other than occasional skin irritation at the site where the chest patches were taped. Collection of blood samples were done by standardized protocol and by qualified professionals and presented minimal risk or discomfort to participants. There were no known legal, psychological, or social risks involved.

Subjects were fully informed of identified procedures prior to enrollment in the study and again at retesting. Additionally, they were informed of potential risks and benefits and signed an informed consent form. Opportunity was given to read the form or have it read to them by the PI. Participants were assured that individual data would be kept confidential. To insure anonymity of participants and confidentiality of data, participants were assigned a study number and collected data was entered on a computer spreadsheet by that number. Original data collection sheets with the matching assigned study number were maintained in a locked security cabinet accessible only by the PI or appropriate faculty representative. Participants were informed that if study results suggested a possible health problem which might need immediate attention, they would be notified and asked if they wanted that information released to their provider. They were further instructed that their participation in the study was voluntary, and they could withdraw from the study at any time with no repercussions, although notification of withdrawal to the PI was encouraged.

\section{Analysis}

Data were analyzed using JMP® Statistical Discovery Software (SAS Institute, 1995). Classification and summarization of sample demographics were calculated through use of descriptive statistics. Means, standard deviations, and ranges were calculated for 
chronological age, age at menarche and menopause, and pre- and post-test physiological measurements of weight, height, BMI, and blood pressure. Additional descriptive statistical values were obtained for sample characteristics including race, education, marital and employment status, presence of hypertension or diabetes, smoking status, exercise status, relevant family history, reproductive history, and characteristics of participants based upon the American Heart Association's cardiovascular risk factors.

Aim one, describe HRV in postmenopausal women, was addressed by performing descriptive analyses for group mean data on each individual HRV value to obtain means, standard deviations, and ranges of scores at baseline and following six weeks of hormone replacement therapy. These values were compared to mean HRV values for healthy female controls (Cashion, 1988).

Aim two was to describe the differences in HRV in postmenopausal women before and after six weeks of hormone replacement therapy. Assuming a normal distribution of variables, paired t-tests were performed on pre- and post-test HRV mean values. Statistical significance of alpha 0.05 established statistical significance with alpha values $\leq 0.10 \geq$ 0.05 used to indicate a statistical trend.

Aim three was to describe $\mathrm{HRV}$ relative to selected cardiovascular risk factors before and after six weeks of hormone replacement therapy. Pearson product-moment or Spearman's Rho correlation coefficients were calculated on selected factors to measure the strength of linear relationship between each factor and each HRV variable. Paired t-tests were done to investigate differences between pre- and post-tests HRV mean values across race, presence/absence of hypertension, and years of menopause. 
Study Assumptions

The assumptions for this study were embedded in the philosophical framework described in chapter one. The following assumptions were the impetus for the evolution of and, ultimately, implementation for this investigation:

1. Menopause is a multi-dimensional event which marks a biological transition in women's natural process of aging. It can be investigated under a biomedical paradigm as a reproductive hormonal deficiency.

2. Determining the definitive nature of reproductive hormone effects on women is difficult because of the many conflicting factors which concurrently affect both the physiological and psychological response to the menopausal event.

3. Considering both the benefits and risks, hormone replacement therapy is a highly effective therapeutic intervention in treating cardiovascular symptomatology, preventing cardiovascular morbidity, and reducing cardiovascular mortality caused by the decrease in reproductive hormones.

4. The frequency and time domains of heart rate variability are relevant measures to assess cardiovascular morbidity and predict CV mortality.

5. Six weeks of HRT will alter a response in heart rate variability because of its attenuation of pressor and neurohormonal responses and its alteration of sympathetic control (Collins \& Beale, 1996).

\section{Study Limitations}

A major limitation to this study was recruitment of participants. For individuals in some of the geographical locations, transportation to the enrollment site to initiate testing 
was a limiting factor for participation. Many did not drive and depended on family members or friends to transport them to the clinic. The occurrence of several major holidays during the data collection period also hindered recruitment. Committing to six weeks of study participation and the need to obtain transportation back to the clinic for retesting could have been a deterring factor in the decision to participate.

Subjects were primarily recruited from individuals who presented to clinics for healthcare intervention. Participants were considered "healthy" at the time of their visit, returning for medication refills or follow-up on their chronic illnesses rather than for an acute, episodic illness. Although they were representative of a clinically-relevant population within a family practice setting, results can not be generalized to the whole population of menopausal women, nor to all PMW who are treated at a community-based clinic. Additionally, the small sample size limits the use of inferential statistics in the analyses and, additionally, generalization of the results.

The drug treatment intervention caused side effects such as break-through vaginal bleeding and breast tenderness for some participants. For two individuals, these side effects caused them to withdraw from the study. Additionally, several women did not comply with the instructions to mark the Drug Documentation Calendar. Therefore, report of compliance was by self-report of memory recall or the count of remaining tablets.

Minimal documentation was available from the literature to determine the length of time needed for an effect of HRT to be observed on HRV. The drug intervention protocol chosen for this study was for six weeks, based on results from a previous study by Collins and Beale (1996) showing neurohormonal changes and pressor responses following six weeks of HRT administration. This time frame may not have been an adequate amount of time to determine if a significant effect on HRV may result in response to HRT.

Control of intervening factors such as age and concomitant disease was done by recording individual participant factors and including the presence or absence of selected 
factors during the study analyses. Medications which might effect cardiac autonomic function were also recorded. However, other intervening factors such as new stresses occurring during the study period could not.

The small size, enrollment of PMW with a large number of confounding variables such as multiple CV risk factors and chronic disease, and use of an exploratory design with multiple analyses considering a significant trend using a $p$-value $=\leq .10$, limit interpretation of results and require a cautious review of findings. 


\section{CHAPTER IV}

\section{RESULTS}

The purpose of this study was to investigate the effect of postmenopausal hormone replacement therapy on cardiac autonomic function. This chapter presents the results of this investigation which utilized an experimental pre-test/post-test design to explore the effects of six weeks of hormone replacement therapy (estrogen/progesterone combined continuous therapy) on heart rate variability. Twenty-six postmenopausal women between the ages of 45-60 were recruited with 24 women completing the six week study.

Three specific aims were identified for conducting this study: (1) describe heart rate variability for postmenopausal women, ages 45-60; (2) describe the differences in heart rate variability for postmenopausal women before and after six weeks of hormone replacement therapy; and, (3) describe heart rate variability for postmenopausal women relative to selected cardiovascular risk factors before and after six weeks of hormone replacement therapy.

Descriptive data which include demographic, medical, and social characteristics of the sample are presented first, followed by further descriptive analyses of pre-and post-test HRV group means. Paired t-tests were used to compare differences between mean values for pre- and post-tests. Using Pearson product-moment or Spearman Rho correlation coefficients, relationships across cardiovascular risk factors were investigated, and paired t-tests were conducted to further investigate differences between pre- and post-test for the total sample across selected cardiovascular risk factors.

Comparison of mean HRV values for PMW participating in this study were made with mean HRV values for healthy female controls. Testing and data analyses for the control group and for this current study were conducted at the Physiologic Function 
Laboratory at a Mid-South research facility. Validity and reliability studies conducted by Cashion (1998) have provided normative data for HRV values within healthy controls and identified values which are borderline and abnormal. Healthy female controls, a subset of the total sample $(\mathrm{N}=158)$, were used as comparison to PMW in this study. This group included $28(24 \%)$ Blacks, $86(74 \%)$ Whites, and $3(2 \%)$ others with a mean sample age of $35.6 \pm 11.8$. Normative data for the total sample are included in Appendix G.

Further statistical analyses were conducted to examine the differences in HRV group means across the various cardiovascular risk factors. However, because of the exploratory design of this study and the small sample size, use of inferential statistics was limited and results of further analyses should be reviewed and interpreted with caution. These analyses were not included in the results chapter, but can be reviewed in Appendices $\mathrm{H}-\mathrm{J}$.

\section{Sample Description}

Tables two through seven present a description of the study participants across demographic, medical, social, reproductive history, and physical characteristics. Additionally, participants are described according to their cardiovascular risk factors as

defined by the American Heart Association. Data are categorized by sample and across race to provide a broader description of participants.

\section{$\underline{\text { Demographic Data }}$}

The demographic data for the sample are presented in Table 2. The 24 women ranged in age from 45-60, mean age of 54.92, with 15 (63\%) Blacks and $9(37 \%)$ Whites. 
Table 2

Demographic Characteristics of Postmenopausal Women

\begin{tabular}{|c|c|c|c|c|c|c|c|c|c|c|}
\hline & \multirow[t]{2}{*}{ Variable } & \multicolumn{3}{|c|}{ Sample $(\mathrm{N}=24)$} & \multicolumn{3}{|c|}{ Blacks $(n=15)$} & \multicolumn{3}{|c|}{ Whites $(n=9)$} \\
\hline & & mean & $\mathrm{n}$ & $\%$ & mean & $\mathrm{n}$ & $\%$ & mean & $\mathrm{n}$ & $\%$ \\
\hline \multirow[t]{5}{*}{ Age } & Mean & 54.9 & & & 54.27 & & & 54.92 & & \\
\hline & SD & 4.45 & & & 4.15 & & & 4.45 & & \\
\hline & Range & $45-$ & & & $47-60$ & & & $45-60$ & & \\
\hline & $<55$ & & 10 & 42 & & 8 & 53 & & 2 & 22 \\
\hline & $\geq 55$ & & 14 & 58 & & 7 & 47 & & 7 & 78 \\
\hline
\end{tabular}

Educational Status

\begin{tabular}{|c|c|c|c|c|c|}
\hline $\begin{array}{l}\text { No High } \\
\text { School } \\
\text { (HS) } \\
\text { Diploma }\end{array}$ & 10 & 42 & 8 & 53 & 2 \\
\hline $\begin{array}{l}\text { Completed } \\
\text { HS* }\end{array}$ & 14 & 58 & 7 & 47 & 7 \\
\hline
\end{tabular}

Marital Status

$\begin{array}{lcccccc}\text { Not Married } & 15 & 63 & 12 & 80 & 3 & 33 \\ \text { Married } & 9 & 47 & 3 & 20 & 6 & 67\end{array}$

Employment Status

$\begin{array}{lcccccc}\text { Unemployed } & 9 & 38 & 5 & 33 & 4 & 5 \\ \text { Employed } & 15 & 62 & 10 & 67 & 44 & 56 \\ \begin{array}{l}\text { Full or Part } \\ \text { Time }\end{array} & & & & & & \end{array}$

* Three women in this group had completed college. 


\section{Medical/Social History}

Current medical and social characteristics are identified in Table 3. Seventeen (71\%) women presented with concomitant disease, including coronary artery disease (1-4\%), diabetes mellitus (8-33\%), and hypertension (11-46\%). Four (17\%) of the women presented with both diabetes mellitus and hypertension. Three (13\%) women were diagnosed with hypothyroidism. Only 7 (29\%) women were on no prescribed medications. Other women were taking a variety of prescribed drugs for disease management including insulin $(n=4)$, oral sulfonylureas $(n=2)$, antihypertensives (calcium channel blockers, $n=2$; beta blockers, $n=1$; diuretics, $n=3$; ACE inhibitors, $n=7$; vasodilators, $n=2$ ), antidepressants $(n=4)$, hypolipidemics $(n=3$, and thyroid supplements $(n=3)$. Nine $(38 \%)$ women were prescribed a multi-drug regimen such as antihypertensive, hypolipidemic, and oral sulfonylurea.

Social history was obtained by self report on the demographic form. Nine (37\%) of the women were current smokers. Of the $15(63 \%)$ non-smokers, 4 had smoked in the past, but all had been non-smokers for $>17$ years. A larger percentage of Caucasian women (44\%) compared to Black women (33\%) were current smokers. However, Caucasian women (67\%) reported more involvement in planned exercise than did Black women (47\%). Intake of alcohol was denied by $13(54 \%)$ of the women. Of those women who reported alcohol intake, 7 (29\%) identified their intake as occasional, and 4 (17\%) identified their intake as frequent.

Exercise was defined in the study as planned physical activity such as walking or bicycle riding, in contrast to unplanned activity which resulted during employment, housework, or gardening, or minimal/no physical activity. Of the 24 women, 13 (54\%) reported that they engaged in planned physical activity compared to $11(36 \%)$ who denied 
participation in planned physical activity. Of the 11 women who denied participation, 8 were employed outside the home.

Review of self-reported family medical history identified 8 (33\%) women reporting family members with cardiovascular disease, 14 (58\%) with diabetes mellitus, and 11 $(46 \%)$ with hypertension. Black women reported a higher percentage of family history of HTN (47\% compared to Whites, 33\%), while Whites reported a higher percentage of family history of coronary artery disease (56\% compared to Blacks, $27 \%$ ). 
Table 3

Medical and Social Characteristics of Postmenopausal Women

\begin{tabular}{lccccccc}
\hline \multicolumn{1}{c}{ Variable } & \multicolumn{2}{c}{$\begin{array}{c}\text { Sample } \\
(\mathrm{N}=24)\end{array}$} & \multicolumn{2}{c}{$\begin{array}{c}\text { Blacks } \\
(\mathrm{n}=15)\end{array}$} & \multicolumn{2}{c}{$\begin{array}{c}\text { Whites } \\
(\mathrm{n}=9)\end{array}$} \\
& $\mathrm{n}$ & $\%$ & $\mathrm{n}$ & $\%$ & \multicolumn{2}{c}{$\mathrm{n}$} & $\%$ \\
\hline $\begin{array}{l}\text { Medical History Variables } \\
\text { Diabetes }\end{array}$ & 8 & 33 & 5 & 33 & 3 & 33 \\
No Diabetes & 16 & 67 & 10 & 67 & 6 & 67 \\
Hypertension & 11 & 46 & 9 & 60 & 2 & 22 \\
No Hypertension & 13 & 54 & 6 & 40 & 7 & 78 \\
Diabetes and Hypertension & 4 & 17 & 4 & 27 & 0 & 0 \\
Coronary Artery Disease & 1 & 4 & 0 & 0 & 1 & 11
\end{tabular}

Social History Variables

$\begin{array}{lcccccc}\text { Current Smoker } & 9 & 37 & 5 & 33 & 4 & 44 \\ \text { Non-Smoker/Quit > 10 yrs } & 15 & 63 & 10 & 67 & 5 & 56 \\ \text { Alcohol Intake: None } & 13 & 54 & 8 & 53 & 5 & 56 \\ \text { Alcohol Intake: Occasionally } & 7 & 29 & 5 & 33 & 3 & 33 \\ \text { Alcohol Intake: Frequently } & 4 & 17 & 2 & 14 & 2 & 21 \\ & & & & & & \\ \text { Planned Exercise } & 13 & 54 & 7 & 47 & 6 & 67 \\ \text { No Planned Exercise } & 11 & 36 & 8 & 53 & 3 & 33\end{array}$

Family History Variables

\begin{tabular}{lcccccc} 
Coronary Artery Disease & 8 & 33 & 4 & 27 & 5 & 56 \\
Diabetes & 14 & 58 & 9 & 60 & 5 & 56 \\
Hypertension & 11 & 46 & 7 & 47 & 3 & 33 \\
\hline
\end{tabular}




\section{$\underline{\text { Reproductive History }}$}

Characteristics of the sample by reproductive history are summarized in Table 4. The mean age of menarche in the sample was 12.5 years $(\mathrm{SD}=0.93)$ with no difference across race. Mean age at menopause was 46.4 years $(\mathrm{SD}=5.99$; $\mathrm{Range}=32-55)$ which is lower than the national average of 51 years. Seventeen (71\%) women experienced menopause at an age less than 51 years. Three of these women experienced premature menopause at an age less than 40 years (Blacks, $n=2$; Whites, $n=1)$. Seven $(29 \%)$ women reported age at menopause between 51-55 years $(\mathrm{Mean}=52.4 ; \mathrm{SD}=1.4)$. The mean age for onset of menopause was the same across race.

The mean number of years of menopause for all women during study participation was 8.64 years $(\mathrm{SD}=5.35$; Range $=1-21$ years). Thirteen $(54 \%)$ women were menopausal less than nine years (Mean=4.86; $\mathrm{SD}=2.48 ;$ Range $=1-8$ years), and $11(46 \%)$ women were menopausal nine years or greater (Mean=13.45; $\mathrm{SD}=3.88$; Range=9-21 years). A difference was observed across race for this variable. Blacks in this sample averaged 7.93 $(\mathrm{SD}=5.71$; Range $=2-21$ years) years of menopause compared to the average number of years of menopause for Whites of 9.89 years ( $\mathrm{SD}=5.09$; Range=1-19 years).

Of the 24 women participating in the study, 13 (54\%) women reported no previous use of hormone replacement therapy (HRT). Eleven (44\%) women reported previous use of HRT, with nine (38\%) women having stopped HRT between three to six months prior to study participation, and two (8\%) women who completed an eight week wash-out period of no HRT use prior to study enrollment.

The inclusion criteria for study participation was based upon having a Follicle Stimulating Hormone (FSH) level of $>30 \mathrm{mIU} / \mathrm{ml}$ and Estradiol level $<30 \mathrm{pg} / \mathrm{ml}$. The mean FSH level for the total sample was 61.91 ( $\mathrm{SD}=21.32)$, and the mean Estradiol level was $5.32(\mathrm{SD}=7.49)$. 
Table 4

Characteristics of Postmenopausal Women by Reproductive History

\begin{tabular}{|c|c|c|c|c|c|c|c|c|c|}
\hline \multirow[t]{2}{*}{ Variable } & \multicolumn{3}{|c|}{ Sample $(\mathrm{N}=24)$} & \multicolumn{3}{|c|}{ Blacks $(n=15)$} & \multicolumn{3}{|c|}{ Whites $(n=9)$} \\
\hline & Mean & $\mathrm{n}$ & $\%$ & Mean & $\mathrm{n}$ & $\%$ & Mean & $\mathrm{n}$ & $\%$ \\
\hline \multicolumn{10}{|l|}{ Age at Menarche } \\
\hline Mean & 12.5 & & & 12.6 & & & 12.33 & & \\
\hline SD & 0.93 & & & .91 & & & 1.0 & & \\
\hline Range & $11-15$ & & & $11-15$ & & & $11-14$ & & \\
\hline \multicolumn{10}{|l|}{ Age at Menopause } \\
\hline Mean & 46.42 & & & 46.53 & & & 46.22 & & \\
\hline $\mathrm{SD}$ & 5.99 & & & 5.7 & & & 6.82 & & \\
\hline Range & $32-55$ & & & $35-55$ & & & $32-53$ & & \\
\hline$<$ age $51^{1}$ & & 17 & 71 & & 12 & 80 & & 5 & 56 \\
\hline$\geq$ age 51 & & 7 & 29 & & 3 & 20 & & 4 & 44 \\
\hline \multicolumn{10}{|l|}{$\begin{array}{l}\text { Years of } \\
\text { Menopause }\end{array}$} \\
\hline Mean & 8.64 & & & 7.93 & & & 9.89 & & \\
\hline SD & 5.35 & & & 5.71 & & & 5.09 & & \\
\hline Range & $1-21$ & & & $2-21$ & & & $1-19$ & & \\
\hline$<9$ years & 4.86 & 13 & 54 & & 10 & 67 & & 3 & 33 \\
\hline$\geq 9$ years & 13.45 & 11 & 46 & & 5 & 33 & & 6 & 67 \\
\hline \multicolumn{10}{|c|}{ Past History of HRT Use } \\
\hline No Past Use & & 13 & 54 & & 6 & 40 & & 7 & 78 \\
\hline Past Use ${ }^{2}$ & & 11 & 46 & & 9 & 60 & & 2 & 22 \\
\hline
\end{tabular}


Table 4 (continued)

\begin{tabular}{|c|c|c|c|c|c|c|c|c|}
\hline \multirow[t]{2}{*}{ Variable } & \multicolumn{2}{|c|}{ Sample $(N=24)$} & \multicolumn{3}{|c|}{ Blacks $(n=15)$} & \multicolumn{3}{|c|}{ Whites $(n=9)$} \\
\hline & Mean & $\%$ & Mean & $\mathrm{n}$ & $\%$ & Mean & $\mathrm{n}$ & $\%$ \\
\hline \multicolumn{9}{|l|}{ FSH Levels } \\
\hline Mean & 61.91 & & 60.31 & & & 64.57 & & \\
\hline $\mathrm{SD}$ & 21.32 & & 19.03 & & & 25.70 & & \\
\hline Range & 30.73- & 02.89 & $31-95.7$ & & & 30.73 & 02.8 & \\
\hline \multicolumn{9}{|c|}{ Estradiol Levels } \\
\hline Mean & 5.32 & & 4.94 & & & 5.96 & & \\
\hline SD & 7.49 & & 4.90 & & & 10.89 & & \\
\hline Range & $1.51-3$ & .09 & $1.5-15.3$ & & & $2.0-34$ & $99^{3}$ & \\
\hline
\end{tabular}

${ }^{1}$ Three women experienced premature menopause $<$ age $40($ Blacks $=2 ;$ Whites $=1)$.

${ }^{2}$ Two women completed an eight week wash-out period prior to study enrollment .

${ }^{3}$ One woman presented with an estradiol level of 34.09. She was included in the study because her FSH level was 40 and she had been amenorrheic for 7 years. Her HRV levels were consistent with other PMW in the study and below the group mean for each value.

\section{Physical Characteristics}

Tables five and six identify height, weight, Body Mass Index (BMI), and blood pressure and delineation of health risk for this sample based upon BMI . The mean height for the sample was 64.67 inches $(\mathrm{SD}=2.70$; Range=60-71). At enrollment, the mean weight for the sample was 166.92 pounds $(\mathrm{SD}=43.88$; Range=108-281). The mean weight 
for Blacks (169.53; $\mathrm{SD}=50.56)$ was similar to Whites (162.56; $\mathrm{SD}=30.46)$, however the upper limit of the ranges differed by 57 pounds $($ Blacks=281; Whites=224) at pre-test and by 62 pounds at post-test $($ Blacks $=283$; Whites $=221)$. The mean weight at post-test was $167.58(\mathrm{SD}=43.86 ;$ Range=111-283). Nine (37\%) women demonstrated a decrease or no change in their weight. An increase in weight was documented in 15 of the women with an average weight gain for these women of 3.27 pounds.

Body Mass Index was calculated at a mean of $28 \mathrm{~kg} / \mathrm{m}^{2}$ on enrollment $(\mathrm{SD}=7.5$; Range $=18-45$ ) with no significant change at post-test. This value identifies the sample to be at a high health risk based upon weight (Lerman, 1997). Seven (29\%) women presented with a BMI of $<25$ representing a low health risk. Nine $(38 \%)$ women presented with BMI's between 25 and < 30 representing a moderate to high health risk. A very high health risk was identified for eight (33\%) women who presented with a BMI of $\geq 30$ (Blacks, $\mathrm{n}=5$; Whites, $\mathrm{n}=3$ ). Three (13\%) Blacks would be classified as morbidly obese based upon their BMI of $>40$.

The mean systolic blood pressure (SBP) of the sample at enrollment was 132.25 $\mathrm{mm} / \mathrm{Hg}(\mathrm{SD}=19.04$; Range=106-190), and the mean diastolic blood pressure (DBP) was 82.92 mm/Hg (SD=7.80; Range=70-100). Ten (42\%) women (Blacks, $\mathrm{n}=7$; Whites, $\mathrm{n}=3$ ) presented with elevations of systolic or diastolic blood pressures categorized as Stage One Hypertension or greater with a $\mathrm{SBP} \geq 140-159$ or a $\mathrm{DBP} \geq 90-99$. One of these woman (Black, age 60, with both HTN and DM) presented with Stage Three Hypertension with a BP of 190/100. Nine of the women were currently taking antihypertensive medication. Mean systolic/diastolic blood pressures at post-test were 135.67 and $83 \mathrm{~mm} / \mathrm{Hg}$. Seven women presented with elevations of $\mathrm{SBP} \geq 140-159$ or a DBP $\geq 90-99$. The woman with Stage Three HTN presented with a BP of $140 / 90$ at re-testing. 
Table 5

Mean Height, Weight, Body Mass Index, and Blood Pressure Values of

Postmenopausal Women

\begin{tabular}{|c|c|c|c|}
\hline Variable & Sample $(\mathrm{N}=24)$ & Blacks $(n=15)$ & Whites $(n=9)$ \\
\hline \multicolumn{4}{|l|}{ Height (in.) } \\
\hline Mean & 64.67 & 64.53 & 64.89 \\
\hline SD & 2.70 & 2.70 & 2.85 \\
\hline Range & $60-71$ & $60-71$ & $61-68$ \\
\hline \multicolumn{4}{|l|}{ Weight (lb.) } \\
\hline \multicolumn{4}{|l|}{ Pre-Test } \\
\hline Mean & 166.92 & 169.53 & 162.56 \\
\hline SD & 43.48 & 50.56 & 30.46 \\
\hline Range & $108-281$ & $108-281$ & $122-224$ \\
\hline \multicolumn{4}{|l|}{ Post-Test } \\
\hline Mean & 167.58 & 169.93 & 163.67 \\
\hline SD & 43.86 & 51.28 & 30.04 \\
\hline Range & $111-283$ & $111-283$ & $124-221$ \\
\hline \multicolumn{4}{|c|}{ Body Mass Index $\left(\mathrm{kg} / \mathrm{m}^{2}\right)^{1}$} \\
\hline $\begin{array}{c}\text { Pre-Test } \\
\text { Mean }\end{array}$ & 28.00 & 28.6 & 27 \\
\hline SD & 7.50 & 9.11 & 3.84 \\
\hline Range & $18-45$ & $18-45$ & $22-35$ \\
\hline \multicolumn{4}{|l|}{ Post-Test } \\
\hline Mean & 28.08 & 28.73 & 27 \\
\hline SD & 7.72 & 9.48 & 3.46 \\
\hline Range & $18-46$ & $18-46$ & $23-34$ \\
\hline
\end{tabular}


Table 5 (continued)

\begin{tabular}{|c|c|c|c|}
\hline Variable & $\begin{array}{l}\text { Sample } \\
(\mathrm{N}=24)\end{array}$ & Blacks $(\mathrm{N}=15)$ & $\begin{array}{l}\text { Whites } \\
(\mathrm{N}=9)\end{array}$ \\
\hline \multicolumn{4}{|c|}{$\begin{array}{l}\text { Systolic blood Pressure } \\
(\mathrm{mm} / \mathrm{Hg})\end{array}$} \\
\hline \multicolumn{4}{|c|}{ Pre-Test } \\
\hline Mean & 132.25 & 134.13 & 129.11 \\
\hline SD & 19.04 & 20.18 & 17.67 \\
\hline Range & $106-190$ & $110-190$ & $106-158$ \\
\hline \multicolumn{4}{|l|}{ Post-Test } \\
\hline Mean & 135.67 & 137.33 & 132.89 \\
\hline SD & 17.43 & 18.25 & 16.65 \\
\hline Range & $96-170$ & $96-170$ & $112-160$ \\
\hline \multicolumn{4}{|c|}{ Diastolic Blood Pressure (mm/Hg) } \\
\hline & \multicolumn{2}{|c|}{ Pre-Test } & 82.22 \\
\hline SD & 7.80 & 9.37 & 4.52 \\
\hline Range & $70-100$ & $70-100$ & $78-90$ \\
\hline \multicolumn{4}{|l|}{ Post-Test } \\
\hline Mean & 83.00 & 83.47 & 82.22 \\
\hline SD & 8.77 & 10.27 & 5.95 \\
\hline Range & $60-96$ & $60-96$ & 74.94 \\
\hline
\end{tabular}

${ }^{1} \mathrm{BMI}$ is a measure of weight for height. It can be calculated from prepared charts which appropriately evaluate individuals between the ages of 19 and 70. Normal BMI is approximately $22 \mathrm{~kg} / \mathrm{m}^{2}$. A BMI of $\geq 27 \mathrm{~kg} / \mathrm{m}^{2}$ is related to high to extremely high health risk (Lerman, 1997). 
Table 6

Body Mass Index and Health Risk of Postmenopausal Women

\begin{tabular}{|c|c|c|c|c|c|c|c|}
\hline \multirow[t]{2}{*}{$\begin{array}{l}\text { Body Mass } \\
\text { Index }\end{array}$} & \multirow[t]{2}{*}{ Health Risk ${ }^{1}$} & \multicolumn{2}{|c|}{$\begin{array}{l}\text { Sample } \\
(\mathrm{N}=24)\end{array}$} & \multicolumn{2}{|c|}{$\begin{array}{l}\text { Blacks } \\
(\mathrm{n}=15)\end{array}$} & \multicolumn{2}{|c|}{$\begin{array}{l}\text { Whites } \\
(n=9)\end{array}$} \\
\hline & & $\mathrm{n}$ & $\%$ & $\mathrm{n}$ & $\%$ & $\mathrm{n}$ & $\%$ \\
\hline 18 to $<25$ & Low & 7 & 29 & 5 & 33 & 2 & 22 \\
\hline 25 to $<27$ & Moderate & 5 & 21 & 1 & 7 & 4 & 45 \\
\hline 27 to $<30$ & High & 4 & 17 & 3 & 20 & 1 & 11 \\
\hline$\geq 30$ & Very High & 8 & 33 & 6 & 40 & 2 & 22 \\
\hline
\end{tabular}

${ }^{1}$ Body Mass Index is related to morbidity and mortality caused by obesity and is an indicator of health risk.

Source: Lerman, R. H. (1997). Obesity: An escalating problem: Addressing the genetic and environmental factors. Contemporary Internal Medicine, 9, 14-21.

\section{Cardiovascular Risk Factors}

Table seven characterizes the sample by presence of risk factors identified by the American Heart Association. Sixteen (67\%) women (Blacks, n=11; Whites, $n=5$ ), reported a family history of cardiovascular disease, either coronary heart disease or hypertension. Presence of contributing factors were identified in eight (33\%) of the women who had diabetes mellitus and $17(71 \%)$ women who had Body Mass Indexes of > 25 which placed them at or above moderate health risk. Three women were morbidly obese. Nine (38\%) women were identified with modifiable risk factors of smoking, $11(46 \%)$ with hypertension, and 11 (46\%) who were non-exercisers. 
Table 7

Characteristics of Postmenopausal Women Based Upon the American Heart Association's Cardiovascular Risk Factors ${ }^{1}$

\begin{tabular}{|c|c|c|c|c|c|c|}
\hline \multirow[t]{2}{*}{ Variable } & \multicolumn{2}{|c|}{$\begin{array}{l}\text { Sample } \\
(\mathrm{N}=24)\end{array}$} & \multicolumn{2}{|c|}{$\begin{array}{l}\text { Blacks } \\
(\mathrm{n}=15)\end{array}$} & \multicolumn{2}{|c|}{$\begin{array}{l}\text { Whites } \\
(n=9)\end{array}$} \\
\hline & $\mathrm{n}$ & $\%$ & $\mathrm{n}$ & $\%$ & $\mathrm{n}$ & $\%$ \\
\hline \multicolumn{7}{|l|}{ Non-Modifiable } \\
\hline Black & 15 & 63 & 15 & & & \\
\hline $\begin{array}{l}\text { Family History of Cardiovascular } \\
\text { Disease (CAD or HTN) }\end{array}$ & 16 & 67 & 11 & 73 & 5 & 56 \\
\hline \multicolumn{7}{|l|}{ Modifiable } \\
\hline Cigarette Smoking & 9 & 38 & 5 & 33 & 4 & 44 \\
\hline Hypertension & 11 & 46 & 9 & 60 & 2 & 22 \\
\hline Non-Exerciser & 11 & 46 & 8 & 53 & 3 & 33 \\
\hline \multicolumn{7}{|l|}{ Contributing Factors } \\
\hline Diabetes Mellitus & 8 & 33 & 5 & 33 & 3 & 33 \\
\hline Obesity (Body Mass Index > 25) & 17 & 71 & 10 & 67 & 7 & 78 \\
\hline
\end{tabular}

${ }^{1}$ Source: American Heart Association. (1998). Risk factors and coronary heart disease. [On-Line]. Available: http://www.amhrt.org/Heart-and-Stroke-A-Z-Guide/riskfact.html.

\section{Aim One}

Aim one was to describe heart rate variability (HRV) in postmenopausal women between the ages of 45-60. Twenty-four hour ambulatory Holter monitoring was used to collect data for analysis of HRV. The frequency domain values delineate between 
sympathetic and parasympathetic cardiac autonomic function, and the time domain values reflect cardiac autonomic balance. Time domain values of pNN50 and rMSSD further reflect vagal influences within autonomic activity.

The frequency and time domains of HRV for the sample of 24 postmenopausal women are summarized in tables eight and nine using descriptive statistics to identify means and standard deviations for each HRV variable at baseline and following six weeks of hormone replacement therapy. Normative data established from analysis of HRV from healthy female controls (mean age $=35.6 \pm 11.8$ ) tested through the Physiologic Function Laboratory (Cashion, 1998) are included in the tables for comparison of values. In addition, mean HRV values for PMW across race are also summarized.

\section{Pre-Test Heart Rate Variability}

Means for all HRV values were lower for study participants than the HRV means for healthy female controls with all frequency domain mean values less than one SD below the values for healthy female controls. The mean value for Low Hertz within the sample was borderline abnormal, as established by normative values for the Physiologic Function Laboratory (Cashion, 1998). This low value was consistent across race. All other mean values in the frequency domains, and all time domain mean values, were within the normal range established for healthy controls, with pNN50, rMSSD, SDNN, and SDANN mean values within one SD of the mean for healthy female controls. White PMW, when compared to Black PMW, demonstrated the pattern identified by Huikuri et al. (1996) and Rosano et al. (1997) as better parasympathetic mediation represented by a higher High Hertz and a lower Low/High Ratio.

SDNN values of less than $50 \mathrm{~ms}$. have been used as a predictors for cardiovascular mortality. Survivors of acute myocardial infarction had approximately a four-fold increased risk of dying during post-recovery when compared with participants whose SDNN value 
was more than $100 \mathrm{~ms}$ (Kleiger, 1987). In this sample of 24 postmenopausal women, only one Black woman, age 53, non-smoker with hypertension and nine years postmenopause presented with values approaching the critical level of 50. Her pre-test SDNN value was 59 , decreasing to 52 on post-test.

Two PMW presented with all HRV values near or above, the values for healthy female controls. Both were Black, ages 47 and 56, and were postmenopausal less than nine years. The 56 year old woman had concomitant disease of both diabetes mellitus and hypertension. Nine (38\%) PMW, five Blacks and four Whites, demonstrated baseline HRV values which were abnormal based on normative data established for healthy controls. Four (44\%) were smokers and four (44\%) were non exercisers. Seven (78\%) were postmenopausal $\geq 9$ years. 
Table 8

Pre-Test Mean Values for Heart Rate Variability for Postmenopausal Women

\begin{tabular}{|c|c|c|c|c|c|}
\hline \multicolumn{2}{|c|}{ HRV Values } & $\begin{array}{l}\text { Mean Values } \\
\text { for Healthy } \\
\text { Controls }^{1}\end{array}$ & $\begin{array}{l}\text { Sample } \\
(\mathrm{N}=24)\end{array}$ & $\begin{array}{l}\text { Blacks } \\
(\mathrm{n}=15)\end{array}$ & $\begin{array}{l}\text { Whites } \\
(n=9)\end{array}$ \\
\hline \multicolumn{6}{|c|}{ Frequency Domains } \\
\hline \multirow[t]{2}{*}{ Total Hertz } & Mean & 7.16 & 6.22 & 6.18 & 6.29 \\
\hline & SD & 0.61 & 0.80 & 0.08 & 0.08 \\
\hline \multirow[t]{2}{*}{ Low $\mathrm{Hz}$} & Mean & 6.31 & 5.15 & 5.15 & 5.16 \\
\hline & SD & 0.64 & 0.89 & 0.09 & 0.09 \\
\hline \multirow[t]{2}{*}{ High Hz } & Mean & 5.18 & 4.11 & 4.07 & 4.17 \\
\hline & $\mathrm{SD}$ & 0.88 & 1.07 & 1.09 & 1.09 \\
\hline \multirow[t]{2}{*}{ Lo/Hi Ratio } & Mean & & 1.30 & 1.31 & 1.29 \\
\hline & $\mathrm{SD}$ & & 0.22 & 0.19 & 0.27 \\
\hline \multicolumn{6}{|c|}{ Time Domains } \\
\hline \multirow[t]{2}{*}{ pNN50 } & Mean & 10.80 & 3.80 & 3.65 & 4.11 \\
\hline & SD & 8.12 & 3.92 & 3.96 & 4.00 \\
\hline \multirow[t]{2}{*}{ rMSSD } & Mean & 32.00 & 20.67 & 20.20 & 21.57 \\
\hline & SD & 13.00 & 8.21 & 8.27 & 7.99 \\
\hline \multirow[t]{2}{*}{ SD } & Mean & 56.00 & 38.00 & 37.93 & 38.07 \\
\hline & SD & 14.00 & 13.18 & 14.86 & 13.67 \\
\hline \multirow[t]{2}{*}{ SDNN } & Mean & 129.00 & 101.92 & 103.93 & 100.79 \\
\hline & SD & 31.00 & 27.19 & 32.24 & 22.38 \\
\hline \multirow[t]{2}{*}{ SDANN } & Mean & 115.00 & 93.17 & 95.27 & 91.71 \\
\hline & $\mathrm{SD}$ & 31.00 & 24.88 & 29.57 & 19.03 \\
\hline
\end{tabular}

${ }^{1}$ Based on data from Physiologic Function Laboratory testing. Sample $=118$ women, mean age $=35.6 \pm 11.8$; range: $18-61$ years. Blacks $=24 \%$; Whites $=74 \%$ Source: Cashion, A. (1998). Twenty-four hour heart rate variability measures in healthy controls. Unpublished raw data, University of Tennessee, Memphis. 
$\underline{\text { Post-Test Heart Rate Variability }}$

The frequency and time domains for heart rate variability collected on post-test following six weeks of postmenopausal hormone replacement therapy are summarized in Table 9. Mean post-test values for PMW remained lower than mean values for healthy female controls, but were within the normal mean ranges for HRV (see Appendix G). Posttest mean values improved over pre-test mean values for Total Hertz, Low Hertz, High Hertz, pNN50, rMSSD, and SD. Frequency domain values of Total Hertz and time domain values of pNN50 and rMSSD were at or within one SD from the mean. Mean values for SDNN and SDANN decreased from pre-test mean values, but were at or above one SD from the mean value for healthy female controls. Low Hertz, High Hertz, and SD remained below one SD from the mean values for healthy female controls. Mean value for Low Hertz improved from borderline abnormal to within the range of normal as established by normative data for healthy controls.

Black PMW demonstrated improvement in seven of the nine HRV values. SDNN and SDANN decreased as was reflected in total sample means, but values were within one SD of the mean for healthy controls. Black PMW reflected an improvement in parasympathetic mediation with an increase in High Hertz and a decrease in Low/High Ratio. In contrast, White PMW improved in only three variables, Low Hertz, pNN50, and SD. Decreases in total sample mean values were noted for Total Hertz and High Hertz, with an increase in Low/High Ratio. SD improved minimally, but rMSSD, SDNN, and SDANN all decreased from pre-test values, with SDNN decreased below one SD from the mean for healthy controls.

Seven (29\%) women (Blacks, $n=4$; Whites, $n=3$ ) demonstrated individual post-test values which were abnormal compared to normative data for healthy controls. Five (71\%) of these women were $\geq 55$ years of age, suggesting an age effect. Additionally, five (71\%) 
were smokers and four (57\%) were non-exercisers by self-report. Five (71\%) of the women had been postmenopausal $\geq 9$ years.

Two women had low SDNN values (58 and 56) on post-test. SDNN is a predictor of cardiac mortality in survivors of acute myocardial infarction when below $50 \mathrm{~ms}$. Only one of these women had a low value (59) on pre-test. The other woman, White, smoker with diabetes, seven years postmenopausal, decreased in SDNN value from 78 on pre-test to 56 on post-test. 
Table 9

Post-Test Mean Values for Heart Rate Variability for Postmenopausal Women

\begin{tabular}{|c|c|c|c|c|c|}
\hline \multicolumn{2}{|c|}{ HRV Values } & $\begin{array}{l}\text { Mean Values } \\
\text { for Healthy } \\
\text { Controls }^{1}\end{array}$ & $\begin{array}{l}\text { Sample } \\
(\mathrm{N}=24)\end{array}$ & $\begin{array}{l}\text { Blacks } \\
(\mathrm{n}=15)\end{array}$ & $\begin{array}{l}\text { Whites } \\
(\mathrm{n}=9)\end{array}$ \\
\hline \multicolumn{6}{|c|}{ Frequency Domains } \\
\hline \multirow[t]{2}{*}{ Total Hertz } & Mean & 7.16 & 6.38 & 6.49 & 6.19 \\
\hline & $\mathrm{SD}$ & 0.61 & 0.78 & 0.64 & 0.98 \\
\hline \multirow[t]{2}{*}{ Low Hz } & Mean & 6.31 & 5.39 & 5.47 & 5.26 \\
\hline & $\mathrm{SD}$ & 0.64 & 0.82 & 0.69 & 1.04 \\
\hline \multirow[t]{2}{*}{ High $\mathrm{Hz}$} & Mean & 5.18 & 4.21 & 4.40 & 3.90 \\
\hline & SD & 0.88 & 1.07 & 0.91 & 1.28 \\
\hline \multirow[t]{2}{*}{ Lo/Hi Ratio } & Mean & & 1.33 & 1.27 & 1.42 \\
\hline & $\mathrm{SD}$ & & 0.23 & 0.19 & 0.28 \\
\hline \multicolumn{6}{|c|}{ Time Domains } \\
\hline \multirow[t]{2}{*}{ pNN50 } & Mean & 10.80 & 4.55 & 4.47 & 4.69 \\
\hline & $\mathrm{SD}$ & 8.12 & 5.15 & 4.72 & 6.10 \\
\hline \multirow[t]{2}{*}{ rMSSD } & Mean & 32.00 & 21.83 & 22.13 & 21.33 \\
\hline & $\mathrm{SD}$ & 13.00 & 10.16 & 8.99 & 12.45 \\
\hline \multirow[t]{2}{*}{ SD } & Mean & 56.00 & 39.46 & 40.27 & 38.11 \\
\hline & $\mathrm{SD}$ & 14.00 & 12.73 & 12.03 & 14.47 \\
\hline \multirow[t]{2}{*}{ SDNN } & Mean & 129.00 & 98.38 & 100.40 & 95.00 \\
\hline & SD & 31.00 & 27.27 & 30.99 & 20.92 \\
\hline \multirow[t]{2}{*}{ SDANN } & Mean & 115.00 & 88.58 & 91.00 & 84.56 \\
\hline & SD & 31.00 & 26.61 & 30.79 & 18.64 \\
\hline
\end{tabular}

${ }^{1}$ Based on data from Physiologic Function Laboratory testing. Sample $=118$ women, mean age $=35.6 \pm 11.8 ;$ range: $18-61$ years. Blacks $=24 \%$; Whites $=74 \%$

Source: Cashion, A. (1998). Twenty-four hour heart rate variability measures in healthy controls. Unpublished raw data, University of Tennessee, Memphis. 
Aim Two

Aim two was to describe the differences in heart rate variability for postmenopausal women before and after six weeks of hormone replacement therapy. A summary of changes in mean HRV values between pre- and post-testing is given in Tables 10 and 11. Paired t-tests were used to compare differences between the means which showed increases in post-test values over pre-test values for seven of the variables. Although no increase was statistically significant at the $\mathrm{p} \leq 0.05$ level, both Low Hertz $(\mathrm{p}=0.06)$ and pNN50 $(p=0.10)$ had increases in mean values which approached significance $(p=\leq 0.10>0.05)$.

Across race, significant differences were observed between pre- and post-test mean values. For Black PMW, significant differences $(p=\leq 0.05)$ were observed for Total Hertz, Low Hertz, High Hertz, and rMSSD. Although not significant, improvement was also noted for pNN50 and SD. Improvement in parasympathetic mediation was observed by noting the increase in High Hertz and the decrease from 1.31 to 1.27 in the Low/High Ratio. No significant improvement were observed across post-test HRV values for White PMW. In contrast to Black PMW, White women significantly $(\mathrm{p}=\leq 0.05)$ increased in Low/High Ratio and decreased in High Hertz, demonstrating poorer parasympathetic mediation and higher sympathetic activity when compared to pre-test values. 
Table 10

Differences Between Pre- and Post-Test Mean Values for Heart Rate Variability for Postmenopausal Women

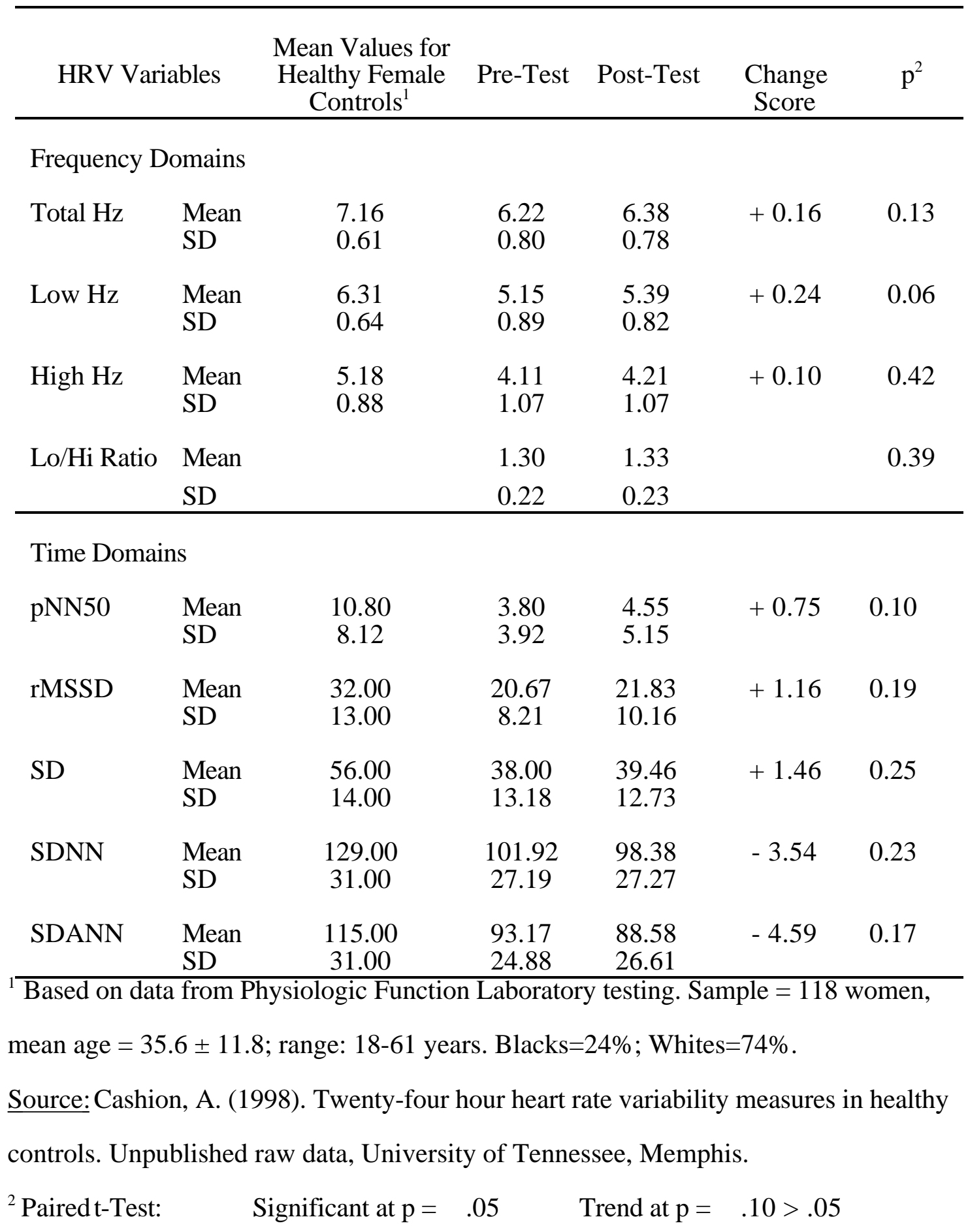


Table 11

Differences Between Pre- and Post-Test Mean Values for Heart Rate Variability for Postmenopausal Women Between and Within Demographic Variable of Race

\begin{tabular}{|c|c|c|c|c|c|c|c|}
\hline \multicolumn{2}{|c|}{ HRV Variables } & \multicolumn{6}{|c|}{ Demographic Variables } \\
\hline & & \multicolumn{3}{|c|}{ Blacks $(\mathrm{n}=15)$} & \multicolumn{3}{|c|}{ Whites $(\mathrm{n}=9)$} \\
\hline & & Pre-Test & Post-Test & $\mathrm{p}^{1}$ & Pre-Test & Post-Test & $\mathrm{p}^{1}$ \\
\hline \multicolumn{8}{|c|}{ Frequency Domains } \\
\hline \multirow[t]{2}{*}{ Total Hz } & Mean & 6.18 & 6.49 & \multirow[t]{2}{*}{.01} & 6.29 & 6.19 & \multirow[t]{2}{*}{$\downarrow .60$} \\
\hline & SD & 0.08 & 0.64 & & 0.08 & 0.98 & \\
\hline \multirow[t]{2}{*}{ Low $\mathrm{Hz}$} & Mean & 5.15 & 5.47 & \multirow[t]{2}{*}{.03} & 5.16 & 5.26 & \multirow[t]{2}{*}{.69} \\
\hline & SD & 0.09 & 0.69 & & 0.09 & 1.04 & \\
\hline \multirow[t]{2}{*}{ High Hz } & Mean & 4.07 & 4.40 & \multirow[t]{2}{*}{.01} & 4.17 & 3.90 & \multirow[t]{2}{*}{$\downarrow .30$} \\
\hline & SD & 1.09 & 0.91 & & 1.09 & 1.28 & \\
\hline $\mathrm{Lo} / \mathrm{Hi}$ & Mean & 1.31 & 1.27 & \multirow[t]{2}{*}{$\downarrow .11$} & 1.29 & 1.42 & \multirow[t]{2}{*}{$\uparrow .05$} \\
\hline Ratio & SD & 0.19 & 0.19 & & 0.27 & .28 & \\
\hline \multicolumn{8}{|c|}{ Time Domains } \\
\hline \multirow[t]{2}{*}{ pNN50 } & Mean & 3.65 & 4.47 & \multirow[t]{2}{*}{.16} & 4.11 & 4.69 & \multirow[t]{2}{*}{.44} \\
\hline & $\mathrm{SD}$ & 3.96 & 4.72 & & 4.00 & 6.10 & \\
\hline \multirow[t]{2}{*}{ rMSSD } & Mean & 20.20 & 22.13 & \multirow[t]{2}{*}{.05} & 21.57 & 21.33 & \multirow[t]{2}{*}{$\downarrow .95$} \\
\hline & SD & 8.27 & 8.99 & & 7.99 & 12.45 & \\
\hline \multirow[t]{2}{*}{ SD } & Mean & 37.93 & 40.27 & \multirow[t]{2}{*}{.12} & 38.07 & 38.11 & \multirow[t]{2}{*}{1.0} \\
\hline & SD & 14.86 & 12.03 & & 13.67 & 14.47 & \\
\hline \multirow[t]{2}{*}{ SDNN } & Mean & 103.93 & 100.40 & \multirow[t]{2}{*}{$\downarrow .36$} & 100.79 & $\downarrow 95.00$ & \multirow[t]{2}{*}{$\downarrow .48$} \\
\hline & SD & 32.24 & 30.99 & & 22.38 & 20.92 & \\
\hline \multirow[t]{2}{*}{ SDANN } & Mean & 95.27 & 91.00 & \multirow[t]{2}{*}{$\downarrow .32$} & 91.71 & $\downarrow 84.56$ & \multirow[t]{2}{*}{$\downarrow .38$} \\
\hline & SD & 29.57 & 30.79 & & 19.03 & 18.64 & \\
\hline
\end{tabular}

${ }^{1}$ Paired t-Test: $\quad$ Significant at $\mathrm{p}=\leq .05$

Trend at $\mathrm{p}=\leq .10>.05$ 
Aim Three

Aim three was to describe heart rate variability for postmenopausal women relative to selected cardiovascular risk factors before and after six weeks of hormone replacement therapy. To measure the strength of linear relationship between heart rate variability values in PMW and their demographic, medical, and social characteristics, Pearson productmoment and Spearman Rho correlation coefficients were calculated. No significant relationships were observed between HRV values and race and age. However an inverse response was seen between all HRV values and increasing age. Across the medical variables, a significant relationship between HTN and Low Hertz/pre-test was found. No other significant relationships were identified between HRV variables and HTN or with diabetes mellitus. Although not significant, inverse relationships were noted between HTN and all HRV values except pNN50 post-test. Also, although not significant, inverse relationships were noted between DM and post-test HRV values.

Inverse relationships were noted between smoking and all HRV values. Significant relationships ( $\mathrm{p}=\leq .10 \geq .05)$ were observed between smoking and post-test HRV values of Total Hertz, Low Hertz, High Hertz, and pNN50. No significant relationships were found across exercise status. Significant inverse relationships or trends were found between years of menopause and all frequency domain values and the time domain value of SD. Table 12.1 summarizes the relationships across selected cardiovascular risk factors and pre- and post-test HRV.

Table 12.2 summarizes tests to investigate differences across race relative to selected cardiovascular risk factors. T-tests or chi square, as appropriate for data, were used. Trend differences at $\mathrm{p}=\leq .10 \geq .05$ were found for HTN $(\mathrm{p}=.07)$.

Table 13 summarizes differences between the pre- and post-test scores across the medical variable of HTN. Eleven PMW (Blacks, $n=9$; Whites, $n=2$ ) reported a diagnosis of 
HTN with pharmacological treatment comprised of varied, and often multiple, antihypertensive drugs (calcium channel blockers, $n=2$; beta blockers, $n=1$; diuretics, $n=3$; ACE inhibitors, $n=7$; vasodilators, $n=2)$. Women with HTN demonstrated significant $(\mathrm{p}=$ $\leq .10 \geq .05$ ) improvement in Low Hertz and the time domain values representing vagal influences of pNN50 and rMSSD. Low Hertz increased from an abnormal value, and Total Hertz, although not demonstrating significance, increased from an abnormal value to a value within normal range. In contrast, no significant improvement in HRV values were observed in PMW without HTN, although frequency domains, pNN50, and SD demonstrated improvement in post-test values over pre-test.

Across years of menopause, women $(44 \%) \geq 9$ years menopausal (Blacks, $n=5$; Whites, $\mathrm{n}=6)$ demonstrated significant $(\mathrm{p}=\leq .10 \geq .05)$ improvement in Total Hertz, Low Hertz, pNN50, and SD. In contrast, PMW < 9 years demonstrated improvement, but not significantly, in post-test HRV values of frequency domains, pNN50, rMSSD, and SD (see Table 14).

Table 12.1

Relationship Between Pre- and Post-Test Heart Rate Variability for Postmenopausal Women Across Sample Demographic, Medical/Social, and Reproductive History Variables

\begin{tabular}{|c|c|c|c|c|c|c|c|c|c|}
\hline $\begin{array}{c}\text { HRV } \\
\text { Variables } \\
1\end{array}$ & $\underset{2}{\text { Race }}$ & Age & $\underset{2}{\mathrm{DM}}$ & $\mathrm{HTN}_{2}$ & $\begin{array}{c}\text { Smoke } \\
\text { Status } \\
2\end{array}$ & $\begin{array}{l}\text { Exer. } \\
\text { Status } \\
2\end{array}$ & $\underset{3}{\mathrm{BMI}}$ & $\begin{array}{l}\text { Age at } \\
\text { Meno } \\
\text { pause }\end{array}$ & $\begin{array}{l}\text { Yrs of } \\
\text { Meno } \\
3\end{array}$ \\
\hline \multicolumn{10}{|c|}{ Frequency Domains } \\
\hline $\begin{array}{l}\text { Total Hz } 1 \\
\text { Total Hz } 2\end{array}$ & $\begin{array}{l}.06 \\
-.08\end{array}$ & $\begin{array}{l}-.18 \\
-.21\end{array}$ & $\begin{array}{l}.13 \\
-.09\end{array}$ & $\begin{array}{l}-.29 \\
-.21\end{array}$ & $\begin{array}{c}-.18 \\
-.42 * *\end{array}$ & $\begin{array}{l}-.05 \\
.16\end{array}$ & $\begin{array}{l}.29 \\
.06\end{array}$ & $\begin{array}{l}.26 \\
.19\end{array}$ & $\begin{array}{l}-.41 * * \\
-.37 *\end{array}$ \\
\hline $\begin{array}{l}\text { Low } \mathrm{Hz} 1 \\
\text { Low Hz } 2\end{array}$ & $\begin{array}{l}-.02 \\
.01\end{array}$ & $\begin{array}{l}-.26 \\
-.26\end{array}$ & $\begin{array}{l}.16 \\
-.12\end{array}$ & $\begin{array}{l}-.41 * * \\
-.33\end{array}$ & $\begin{array}{l}-.09 \\
-.35^{*}\end{array}$ & $\begin{array}{l}-.11 \\
.21\end{array}$ & $\begin{array}{l}.27 \\
.01\end{array}$ & $\begin{array}{l}.23 \\
.23\end{array}$ & $\begin{array}{l}-.43 * * \\
-.43 * *\end{array}$ \\
\hline $\begin{array}{l}\text { High Hz } 1 \\
\text { High } \mathrm{Hz} 2\end{array}$ & $\begin{array}{r}.06 \\
-.15\end{array}$ & $\begin{array}{l}-.03 \\
-.17\end{array}$ & $\begin{array}{l}.05 \\
-.01\end{array}$ & $\begin{array}{l}-.14 \\
-.08\end{array}$ & $\begin{array}{l}-.21 \\
-.38 *\end{array}$ & $\begin{array}{l}-.07 \\
.03\end{array}$ & $\begin{array}{l}.27 \\
.15\end{array}$ & $\begin{array}{l}.33 \\
.21\end{array}$ & $\begin{array}{l}-.35^{*} \\
-.34 *\end{array}$ \\
\hline
\end{tabular}




\begin{tabular}{lccccccccc} 
Lo/Hi & -.04 & -.18 & .04 & -.11 & .29 & .13 & -.22 & -.26 & .12 \\
Ratio 1 & & & & & & & & & \\
Lo/Hi & .29 & .03 & -.02 & -.21 & .32 & .05 & -.19 & -.21 & .22 \\
Ratio 2 & & & & & & & & & \\
& & & & & & & & & \\
\hline Time Domains & & & & & & & & & \\
pNN50 1 & .14 & -.05 & .04 & -.21 & -.30 & .05 & .18 & .21 & -.27 \\
pNN50 2 & -.12 & .03 & .03 & .02 & $-.36^{*}$ & .03 & .21 & .18 & .14 \\
& & & & & & & & & \\
rMSSD 1 & .08 & -.03 & .01 & -.24 & -.25 & .01 & .13 & .26 & -.31 \\
rMSSD 2 & -.10 & -.02 & -.02 & -.03 & -.31 & -.01 & .18 & .21 & -.22 \\
SD 1 & .08 & -.19 & .01 & -.26 & -.23 & .02 & .12 & .25 & $-.42^{* *}$ \\
SD 2 & -.01 & -.09 & -.01 & -.19 & -.30 & .05 & .05 & .28 & $-.37 *$ \\
& & & & & & & & & \\
SDNN 1 & -.01 & -.23 & -.04 & -.16 & -.09 & -.01 & .26 & .06 & -.26 \\
SDNN 2 & -.02 & -.33 & -.08 & -.27 & -.21 & -.05 & .21 & .01 & -.27 \\
& & & & & & & & & \\
SDANN 1 & -.02 & -.21 & -.01 & -.02 & -.06 & -.03 & .26 & .01 & -.19 \\
SDANN 2 & -.02 & $-.36 *$ & -.06 & -.31 & -.12 & -.11 & .24 & .01 & -.19 \\
\hline
\end{tabular}

${ }^{1}$ Variables are identified as 1 and 2 representing Pre-Test (1) and Post-Test (2).

${ }^{2}$ Spearman Rho Corr.: **Significant at $\mathrm{p}=\leq 0.05 *$ Trend at $\mathrm{p}=\leq 0.10>0.05$.

${ }^{3}$ Pearson's Corr.: $\quad * *$ Significant at $\mathrm{p}=\leq 0.05 \quad *$ Trend at $\mathrm{p}=\leq 0.10>0.05$. 
Table 12.2

Comparisons of Differences Across Race for Selected Cardiovascular Risk Factors

\begin{tabular}{lcccc}
\hline \multicolumn{1}{c}{ Variable } & Test ${ }^{1,2}$ & $\mathrm{p}^{3}$ & $\begin{array}{c}\text { Blacks } \\
(\mathrm{n}=15)\end{array}$ & $\begin{array}{c}\text { Whites } \\
(\mathrm{n}=9)\end{array}$ \\
\hline Age & $-.92^{1}$ & .37 & $\mathrm{M}=54.27$ & $\mathrm{M}=56.0$ \\
Diabetes Mellitus & $.00^{2}$ & 1.0 & $\mathrm{n}=5$ & $\mathrm{n}=3$ \\
Hypertension & $3.38^{2}$ & .07 & $\mathrm{n}=9$ & $\mathrm{n}=2$ \\
Smoking Status & $.29^{2}$ & .59 & $\mathrm{n}=5$ & $\mathrm{n}=4$ \\
Planned Exercise Status & $.92^{2}$ & .34 & $\mathrm{n}=7$ & $\mathrm{n}=6$ \\
BMI & $.50^{1}$ & .62 & $\mathrm{M}=28.6$ & $\mathrm{M}=27$ \\
BMI by Health Risk & $4.88^{2}$ & .18 & & $($ See Table 6) \\
Age at Menopause & $.12^{1}$ & .91 & $\mathrm{M}=46.53$ & $\mathrm{M}=46.22$ \\
Age at Meno $\geq 51$ years & $1.60^{2}$ & .21 & $\mathrm{n}=3$ & $\mathrm{n}=4$ \\
Years of Menopause & $-.84^{1}$ & .41 & $\mathrm{M}=7.93$ & $\mathrm{M}=9.89$ \\
${ }^{3}$ Significant: $\mathrm{p}=\leq .05$ & $2.6^{2}$ & .11 & $\mathrm{n}=5$ & $\mathrm{n}=6$ \\
\hline Tears of Menopause $\geq 9$ years & & & & \\
\hline
\end{tabular}


Table 13

Differences Between Pre- and Post-Test Mean Values for Heart Rate Variability for Postmenopausal Women By the Absence/Presence of Hypertension

\begin{tabular}{|c|c|c|c|c|c|c|c|}
\hline \multirow{3}{*}{\multicolumn{2}{|c|}{ HRV Variables }} & \multicolumn{6}{|c|}{ Medical Variables } \\
\hline & & \multicolumn{3}{|c|}{ Hypertension-Absent $(\mathrm{n}=13)$} & \multicolumn{3}{|c|}{ Hypertension-Present $(\mathrm{n}=11)$} \\
\hline & & Pre-Test & Post-Test & & Pre-Test & Post-Test & $\mathrm{p}^{1}$ \\
\hline \multicolumn{8}{|c|}{ Frequency Domains } \\
\hline \multirow[t]{2}{*}{ Total Hz } & Mean & 6.48 & 6.56 & \multirow[t]{2}{*}{.57} & 5.92 & 6.17 & \multirow[t]{2}{*}{.11} \\
\hline & SD & 0.53 & 0.69 & & 0.96 & 0.86 & \\
\hline \multirow[t]{2}{*}{ Low $\mathrm{Hz}$} & Mean & 5.52 & 5.66 & \multirow[t]{2}{*}{.38} & 4.72 & 5.07 & \multirow[t]{2}{*}{.09} \\
\hline & SD & 0.62 & 0.66 & & 0.98 & 0.91 & \\
\hline \multirow[t]{2}{*}{ High Hz } & Mean & 4.28 & 4.32 & \multirow[t]{2}{*}{.81} & 3.91 & 4.09 & \multirow[t]{2}{*}{.38} \\
\hline & $\mathrm{SD}$ & 0.82 & 1.01 & & 1.31 & 1.17 & \\
\hline $\mathrm{Lo} / \mathrm{Hi}$ & Mean & 1.32 & 1.35 & \multirow[t]{2}{*}{$\uparrow .20$} & 1.28 & 1.30 & \multirow[t]{2}{*}{$\uparrow .79$} \\
\hline Ratio & SD & 0.19 & 0.23 & & 0.26 & 0.25 & \\
\hline \multicolumn{8}{|c|}{ Time Domains } \\
\hline \multirow[t]{2}{*}{ pNN50 } & Mean & 4.40 & 4.53 & \multirow[t]{2}{*}{.83} & 3.09 & 4.58 & \multirow[t]{2}{*}{.03} \\
\hline & SD & 4.27 & 5.70 & & 3.50 & 4.70 & \\
\hline \multirow[t]{2}{*}{ rMSSD } & Mean & 22.69 & 22.62 & \multirow[t]{2}{*}{.96} & 18.27 & 20.91 & \multirow[t]{2}{*}{.02} \\
\hline & $\mathrm{SD}$ & 8.35 & 11.23 & & 7.72 & 9.19 & \\
\hline \multirow[t]{2}{*}{ SD } & Mean & 40.85 & 41.46 & \multirow[t]{2}{*}{.75} & 34.64 & 37.09 & \multirow[t]{2}{*}{.16} \\
\hline & SD & 9.79 & 12.00 & & 16.18 & 13.72 & \\
\hline \multirow[t]{2}{*}{ SDNN } & Mean & 104.62 & 103.08 & \multirow[t]{2}{*}{$\downarrow .69$} & 98.73 & 92.82 & \multirow[t]{2}{*}{$\downarrow .23$} \\
\hline & SD & 24.80 & 24.65 & & 30.70 & 30.31 & \\
\hline \multirow[t]{2}{*}{ SDANN } & Mean & 94.23 & 93.69 & \multirow[t]{2}{*}{$\downarrow .90$} & 91.91 & 82.55 & \multirow[t]{2}{*}{$\downarrow .07$} \\
\hline & $\mathrm{SD}$ & 24.01 & 24.05 & & 26.00 & 29.34 & \\
\hline
\end{tabular}

${ }^{1}$ Paired t-Test: $\quad$ Significant at $\mathrm{p}=\leq .05 \quad$ Trend at $\mathrm{p}=\leq .10>.05$ 
Table 14

Differences Between Pre- and Post-Test Mean Values for Heart Rate Variability for

Postmenopausal Women by Number of Years of Menopause

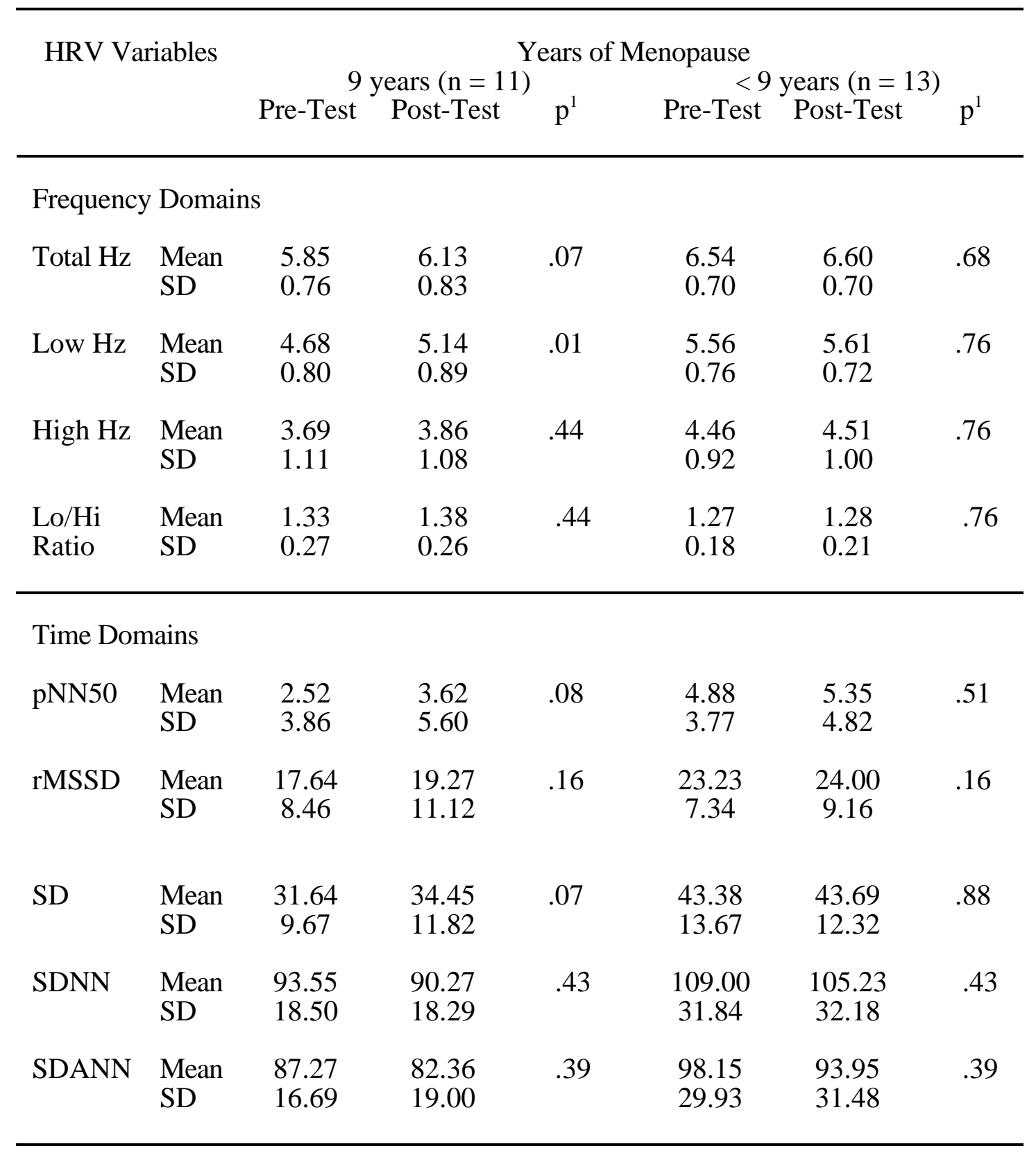

${ }^{1}$ Paired t-Test: $\quad$ Significant at $\mathrm{p}=\leq .05 \quad$ Trend at $\mathrm{p}=\leq .10>.05$ 


\section{CHAPTER V}

\section{DISCUSSION, RECOMMENDATIONS, AND CONCLUSIONS}

A biomedical conceptual framework was used for this study, incorporating the construct of menopause and the two concepts of hormone replacement therapy and heart rate variability. Chapter five provides a discussion on the findings of the study and factors which may have influenced the results and the interpretation of results. A brief overview of the study is given, followed by identification of sample characteristics and methodology, and finally a discussion of each study aim.

The purpose of this study was to examine the effect of postmenopausal hormone replacement therapy on cardiovascular autonomic function (CAF) in women between the ages of 45-60. Analysis of 24-hour heart rate variability was used to quantify CAF and to address three specific study aims:

1. Describe heart rate variability for postmenopausal women, ages $45-60$

2. Describe the differences in heart rate variability for postmenopausal women before and after six weeks of hormone replacement therapy.

3. Describe heart rate variability for postmenopausal women across selected cardiovascular risk factors before and after six weeks of hormone replacement therapy.

To address study aims, an exploratory, pre-test/post-test experimental design with each woman serving as her own control was used. Postmenopausal women between the ages of 45 and 60 (mean age=54.9; $\mathrm{SD}=4.45$ ) were recruited for this study from two community-based primary care clinics and a suburban church. Because previous research on hormone replacement therapy has been criticized for selection bias which might influence positive results, recruitment in these settings provided a diverse population of 
participants with varying ethnic and socio-economic characteristics. Twenty-six women were enrolled in the six week intervention study with twenty-four women (Blacks/n=15, $63 \%$; Whites/n=9, 37\%) completing the post-test following six weeks of hormone replacement therapy.

Inclusion and exclusion criteria for the study were liberal, unlike the related studies by Huikuri et al. (1996) and Rosano et al. (1997) which restricted HRV analysis to women with no documented cardiovascular disease. To provide a clinically relevant population, postmenopausal women from a suburban church $(n=4)$ and two community-based, family practice settings $(n=20)$ were recruited at the time of their visit to the clinic. Although "healthy" at the visit, often for medication refills or follow-up only, these women presented with a variety of concomitant diseases, such as diabetes mellitus (DM), $(n=8,33 \%)$ and hypertension $(\mathrm{HTN}),(\mathrm{n}=11,46 \%)$. One woman had a past history of acute myocardial infarction without complications three months prior to enrollment. Some women had comorbidity such as diabetes mellitus and hypertension $(n=4)$, and $17(71 \%)$ of the women reported regular use of prescription medications. Participants reported a family history of heart disease $(n=8,33 \%), D M(n=14,58 \%)$ and HTN $(n=11,46 \%)$. Unlike the cited studies, the sample included PMW who reported use of nicotine $(\mathrm{n}=9,37 \%)$ and alcohol $(n=11,46 \%)$. Although the sample was representative of PMW who reported to community-based health clinics, use of non-random selection limited generalization of results to the total population of postmenopausal women. The diversity of the group also was a limitation to the study. The small sample size within subgroups hindered the ability to control for multiple variables which feasibly could have influenced heart rate variability.

A second limitation to interpretation of study results was related to the six week protocol for hormone replacement therapy. At the time of this study, no guidelines existed which established the length of time necessary for HRV to respond to the administration of HRT. Therefore, the drug intervention protocol established for this study was based on 
research reported by Collins and Beale (1996) who reported the attenuation of pressor and neurohormonal responses and modulation of sympathetic and parasympathetic influences on the ANS in PMW following six weeks of estrogen. Research by Rosano et al. (1997), however, published during the course of this research, suggested that a longer period of 16 weeks may be necessary to stabilize cardiac autonomic function in symptomatic menopausal women. After six weeks administration of HRT to study participants, varying responses were observed across repeated HRV testing, with most values demonstrating a nonsignificant increase but consistent with results reported in the study by Rosano et al. (1997). However, two time domain values consistently demonstrated decreased values. These findings could be directly related to the limited time interval and use of HRT between pre- and post-tests.

Huikuri et al. (1996) of Finland suggested that vagal responses to cardiovascular autonomic regulation were lower for PMW not on estrogen replacement therapy compared to women taking estrogen. Furthermore, they suggested that hormonal factors may influence changes in autonomic modulation. Unique characteristics of participants in this study which may relate to hormonal factors may also have influenced study results. Participants reported number of years of menopause with sample mean of $8.64 \pm 5.35$ (range $=1-21$ years $).$ Reported age at menopause $(m=46.42 \pm 5.99$ years; range $=32-55)$ was lower than the 51 years reported as average age of menopause in the U.S. This finding may indicate that the PMW in this study have had different reproductive changes and transition into menopause, and may respond to hormones differently because of a greater estrogen deficiency, than their age peers who experienced menopause at a later period in their reproductive life. A possible rationale for differences in HRV values before and after HRT administration could be suggested that HRV values for these women may be more similar to older PMW who experienced menopause at the more typical mean age of 51. Three of the women experienced premature menopause prior to the age of 40; no rationale 
for this occurrence could be isolated and the cause is probably multi-factorial. In addition to low socio-economic status, the demographic characteristics for these three women were: (a) White, smoker, DM; (b) Black, smoker, HTN; (c) Black, non-smoker, DM and HTN. All three women were patients at the community clinic. The effect of premature menopause on HRV has not been investigated but could have influenced study results. Although HRV values for these women varied, the values reflected patterns demonstrated by PMW who had experienced menopause at a later age.

The hormone combination for this study may have influenced results. Current clinical practice guidelines dictate that postmenopausal women with intact uteri be prescribed HRT consisting of estrogen with the addition of a progestin to prevent endometrial hyperplasia (American College of Physicians, 1992; Barrett-Connor, 1995). All women enrolled in the study had intact uteri; therefore, the pharmacological HRT intervention consisted of six weeks of oral conjugated estrogen $0.625 \mathrm{mg}$ and medroxyprogesterone, $2.5 \mathrm{mg}$ given in a single oral medication packaged as PremPro ${ }^{\mathrm{TM}}$, a trademark of Wyeth-Ayerst Laboratories. A potential confounding factor in this study could have resulted from the combination of estrogen and progesterone for HRT versus the effect of estrogen alone on heart rate variability. Conflicting research results indicate that the addition of a progestin may or may not attenuate the positive effects of estrogen (Grady et al, 1992; Notelovitz, 1989). Because current clinical practice guidelines advocate progestin in the HRT regimen for women with intact uteri, the recommended guidelines were followed and the combination used. Compliance to the drug regimen was substantiated by self report and may have influenced results if the protocol was not followed. Although participants were asked to complete and return a Drug Documentation Calendar validating their compliance, only 11 women returned the document. Twenty-two women completing the study verbally acknowledged adherence to the protocol. Two women stated that they had missed taking one pill during the six week study period. It is assumed that this fact had 
no negative effects on their HRV post-test values. Pre-test estradiol levels increased and FSH levels decreased in all women following the six weeks of HRT.

\section{Aim One}

The first aim of the study was to describe heart rate variability (HRV) in postmenopausal women between the ages of 45-60. HRV values determined by twentyfour hour ambulatory Holter monitoring and analysis, and obtained before and after six weeks of HRT administration, were generally within normal range, but lower than the referent values for healthy females. Validity of this conclusion is dependent upon the validity and appropriateness of referent group means and established values for normal, borderline, and abnormal values. For comparison, normative values were based on sample mean HRV values obtained from healthy female controls $(n=118$; mean age $=35.6 \pm 11.8)$ tested in the same laboratory and using the same analyses and testing protocols used in conducting this research. The age of the referent group is approximately ten years less than that of study participants, and the differences between HRV means of these two groups could have been influenced by this age factor. The majority of women in this study also reported concomitant morbidity such as $\operatorname{HTN}(n=11)$ and DM $(n=8)$ and use of prescription medications which also may be factors in influencing decreased values. Normative data based on age, disease status, and postmenopausal status have not been established but would have provided a more relevant referent group.

\section{Pre-Test Heart Rate Variability}

Sample means for all pre-test HRV values were lower for study participants than the HRV means for healthy female controls with all frequency domain mean values less than one SD below the values for healthy female controls. The mean value for Low Hertz 
within the sample was borderline abnormal. This low value was consistent across races. All other mean values in the frequency domains, and all time domain mean values, were found to be within normal range. Time domain values of pNN50, rMSSD, SDNN, and SDANN mean values were within one SD of the mean for healthy female controls. These results were consistent with that of other researchers who suggest an inverse relationship between HRV and age and chronic illness (Cowan et al., 1994; Liao et al., 1995; Omboni et al., 1996; \& van Ravenswaaij-Arts et al., 1993).

Consistent with study results by Liao (1995) investigating HRV across race, descriptive comparisons across race in this study demonstrated nonsignificantly lower HRV values for Black PMW, when compared to White PMW for all frequency domains, pNN50, rMSSD, and SD. White PMW also demonstrated more optimum parasympathetic activity which was reflected in their higher values for High Hertz (Whites=4.17; Blacks=4.07) and lower value for Low/High Ratio (Whites=1.29; Blacks=1.31). Both Blacks and Whites presented with similar cardiovascular risk factors of age ( $\mathrm{p}=.37), \mathrm{DM}$ $\left(\chi^{2}=1\right)$, smoking $\left(\chi^{2}=.29\right)$, lack of exercise $\left(\chi^{2}=92\right)$, BMI $(\mathrm{p}=.62)$, and age at menopause $(\mathrm{p}=.41)$, but differed across presence of $\operatorname{HTN}\left(\chi^{2}=.07\right)$ and years of menopause $\geq 9$ years or $<9$ years $\left(\chi^{2}=.11\right)$.

\section{$\underline{\text { Post-Test Heart Rate Variability }}$}

HRV sample means following six weeks of HRT continued to be lower than norms for healthy female controls, a finding expected because of age and chronic disease differences between the sample and referent group, but remained within normal range. Across race, Black PMW demonstrated an increase in seven of the nine HRV values but also showed a decrease in SDNN and SDANN as reflected by the total sample. A low value for High Hertz with a high value for Low/High Ratio is considered indicative of sympathetic predominance, and a change toward a higher value of High Hertz with a lower 
Low/High Ratio demonstrating a stabilization of the autonomic control of the cardiovascular system (Rosano, 1997). Black PMW demonstrated this change from pretest to post-test after HRT administration. High Hertz increased from 4.07 to 4.40 , and Low/High Ratio decreased from 1.31 to 1.27 reflecting improved balance of mediation between sympathetic/parasympathetic function. White postmenopausal women demonstrated the opposite effect with High Hertz decreasing from 4.17 to 3.90 and Low/High Ratio increasing from 1.29 to 1.42 . The causes of these findings remain speculative, and further research is necessary to determine if HRV responds differently to the administration of HRT when investigated across race. Because of an increased proportion of PMW with HTN for Black women, HTN alone, race alone, or race and HTN together may have strong influence on the change. Years of menopause should have had a positive effect on HRV improvement because Black women, in this sample, had fewer years of menopause than White women, and, therefore, fewer years without endogenous estrogen with its cardiovascular protective effects. Additional studies could help to clarify the relationships between race, HRV, and response of hormone replacement therapy. Because there have been no published studies which have examined the effect of hormone replacement therapy on HRV across racial cohorts, a longitudinal, prospective study with a larger sample size would provide the opportunity to investigate this question.

The finding of decreased values on post-test for time domain measurements of SDNN and SDANN was not expected and had not previously been described in the literature. Because these values are considered good measures of primary autonomic influence on heart rate variability and reflections of circadian rhythmicity, abnormal values may indicate pathology and predict mortality in some individuals. In this sample, the values although decreased, continued to reflect normal values. A study with a larger sample size and extended time frame for HRT administration is necessary to investigate whether or not this trend would remain consistent. If researchers addressing HRV with HRT in PMW 
would report both frequency and time domain values, further investigation into the consistency of this finding could occur.

\section{Aim Two}

Study aim two was to describe the differences in HRV for PMW before and after six weeks of hormone replacement therapy. Paired t-tests were used to compare means between pre- and post-tests for the total sample and by each race. For the total sample, few significant increases in HRV were noted. Increases in Low Hertz $(p=.06)$ and pNN50 $(\mathrm{p}=.10)$ demonstrated trends $(\mathrm{p}=\leq .10>.05)$. Increases, although not significant, were also found for Total Hertz, High Hertz, rMSSD, and SD. On post-test, following six weeks of HRT, nonsignificant decreases in SDNN and SDANN were found. The pattern of pre-test HRV frequency values below one SD of the mean for healthy women, and the time domain values higher and within one SD of the mean, may reflect that HRV time domains could not feasibly increase sufficiently to demonstrate significant values.

White PMW showed no significant differences after six weeks of HRT. However, significant differences were observed between pre- and post-test mean values for Black PMW for Total Hertz ( $\mathrm{p}=.01)$, Low Hertz $(\mathrm{p}=.03)$, High Hertz $(\mathrm{p}=.01)$ and rMSSD $(\mathrm{p}=.05)$. Increases, although not significant, were observed for $\mathrm{pNN} 50(\mathrm{p}=.16)$ and SD ( $\mathrm{p}=.12)$. Low/High Ratio showed an improvement in sympathetic/parasympathetic balance with a decrease $(\mathrm{p}=.11)$ in this HRV value. These preliminary findings suggest that Black PMW may benefit from HRT administration by improved cardiovascular tone related to improvement of parasympathetic function. Improvement in values for Black women occurred despite a greater proportion of women compared to White PMW with HTN and the short period of HRT administration of six weeks. Black women, additionally, had lower HRV values than White women at pre-test. Given that Black females have a two-fold 
risk of coronary death as compared to White women (AHA, 1988), further studies to confirm these findings and conducted on a larger sample size, should be carried out to clarify the benefit of HRT in this population. Hypertension as a CVD risk factor is addressed under aim three.

\section{Aim Three}

Aim three, describe HRV for PMW relative to selected cardiovascular risk factors, was addressed by Pearson's product-moment and Spearman's Rho Correlational analysis of the relationship between HRV and sample demographic, medical, and social characteristics. Although no significant correlation was demonstrated for race and HRV variables, three other risk factors, HTN, smoking, and years of menopause did demonstrate significant associations, primarily in the frequency domains. The AHA risk factors for CVD were originally to be addressed for differences in HRV by dichotomizing the total sample for separate analyses for each risk factor. For Black and White PMW differences were found for HRV values, therefore, comparisons of differences across race for selected cardiovascular risk factors were conducted using t-tests or Chi Square, as appropriate. Because of the sample size and the risk of Type II error resulting from multiple analyses, risk factors for which Black and White PMW did not differ, were not presented. Differences across race was demonstrated for the risk factor of $\operatorname{HTN}\left(\chi^{2}=.07\right)$ with a larger number of Black women $(n=9)$ compared to White women $(n=2)$ self-reporting the presence of HTN. Additional differences were found across years of menopause $\left(\chi^{2}=\right.$ .11) with Black women having fewer years of menopause $(M=7.93)$ when compared to White women $(\mathrm{M}=9.89)$.

Only HTN was found to differ across race approaching a level of significance $(\mathrm{p}=.07)$. Paired t-test analyses of pre- and post-test differences for PMW with HTN 
$(\mathrm{n}=11)$ and for PMW without HTN $(\mathrm{p}=13)$ demonstrated significant improvement for hypertensive women for the vagal values of pNN50 ( $\mathrm{p}=.03)$ and $\operatorname{rMSSD}(\mathrm{p}=.02)$, a trend for Low Hertz ( $\mathrm{p}=.09)$, and nonsignificant improvement for Total Hertz, High Hertz, and SD. No significant differences were found in HRV for PMW without HTN, although Total Hertz, Low Hertz, High Hertz, pNN50, and SD improved in mean values. In contrast to these findings, pre-test differences were observed between the group with/without hypertension. Women with HTN $(\mathrm{n}=11,46 \%)$ demonstrated lower mean values in all domains when compared to postmenopausal women without HTN (n=13; 54\%). A significant $(\mathrm{p}=0.02)$ difference was observed for Low Hertz, approaching significance $(\mathrm{p}=0.09)$ for Total Hertz, and a lower mean for High Hertz, although not significant. These findings suggest a difference in the balance between sympathetic/parasympathetic function in PMW not on hormones with HTN compared to PMW without HTN. The women in the HTN group were taking a variety of different antihypertensive medications, including diuretics, calcium channel blockers, ACE inhibitors, beta blockers, and vasodilators. What influence these pharmacological interventions had on the results requires further investigation in a controlled study which would follow a larger cohort of PMW with and without HTN. Because of the disproportionate number of Blacks $(n=9)$ in comparison to Whites ( $n=2)$, these results have to be interpreted cautiously. It is unknown if the factor influencing the significant improvements was related to race or to disease process because both Black PMW and women with HTN, which was primarily composed of Blacks, demonstrated similar responses for High Hertz and rMSSD. The small sample size limited the use of further multivariate statistical analyses to investigate relative contribution of each risk factor.

Another differing characteristic between Black and White PMW was years of menopause. When the total sample mean $(8.64 ; \mathrm{SD}=5.35)$ was used to dichotomize the sample, chi square analysis indicated differences at 0.11 . PMW with $\geq 9$ years of 
menopause (Blacks, $n=5$; Whites, $n=6$ ) demonstrated lower pre- and post-test HRV values in frequency domains and demonstrated significant or trends for improved values for Total Hertz $(\mathrm{p}=.07)$, Low Hertz $(\mathrm{p}=.01), \mathrm{pNN} 50(\mathrm{p}=.08)$, and SD $(\mathrm{p}=.07)$. No significant improvements were observed for PMW < 9 years menopausal (Blacks, n=10; Whites, $\mathrm{n}=3$ ). Years of menopause have usually been discussed in relation to osteoporosis, addressing duration of hypoestrogenic state or treatment with HRT, in relation to bone density. More recent investigations by researchers have suggested that a potential exists for increasing risk of breast cancer with 10 or more years of estrogen administration. In this study, years of menopause may serve as a surrogate for years of decreasing hormone levels. Consequently, women with more years of menopause may have a greater risk for CVD because of extended time with loss of endogenous estrogen. Chronological age was similar for the two subgroups addressed for years of menopause and significant HRV values. Because research of HRV in PMW has been limited, influences of years of menopause on HRV has not been previously delineated except as a general demographic characteristic.

\section{Conclusions}

Research has provided extensive findings on the beneficial (as well as the deleterious) modulatory effects of estrogen. However, limited research has examined the effect of estrogen on cardiac autonomic function, specifically heart rate variability. Although studies by Huikuri et al. (1996) and Rosano et al. (1997) investigated the effect of hormone replacement therapy on HRV, the characteristics of the population represented in those studies differed from the participants in this study across disease process, pharmacological intervention, and use of HRT regimen. 
The aim for this research was to describe HRV in postmenopausal women before and after six weeks of HRT in a sample of women who live in the community and seek primary health care in a family practice clinical setting. Descriptive data confirmed expected findings that HRV values, because of aging, were lower than laboratory norm values of younger, healthy women, and that improved values after six weeks of HRT continued to be lower. HRV values of PMW with and without CVD risk factors of black race, HTN, and $\geq$ 9 years of menopause, reflected this pattern; women with risk factors generally had lower HRV values. Values low on pre-test, generally in the frequency domains, demonstrated improvement. HRV values improved with six weeks of HRT for PMW with selected risk factors, particularly HTN, and principally, and consistently, for frequency domain values of Total Hertz, Low Hertz, and High Hertz, and to a lesser extent, for time domain values of pNN50 and rMSSD. The decreases in SDNN and SDANN were not anticipated and merit further investigation. Because both of these time domain values reflect long-term variability over 24 hours, differences in HRV may have been decreased by estrogen. However time domain values of pNN50 and rMSSD, reflecting vagal influences, showed increases, not decreases, and Total Hertz found to be highly correlated with SDNN and also a global indicator of HRV status, showed significant $(\mathrm{p}=.01)$ improvement for Black women.

The small sample size, enrollment of women with a large number of confounding variables such as multiple CV risk factors and chronic disease, and the exploratory design of the study limit interpretation of these results and require a cautious review of findings. The significant improvements in HRV for Black women following administration of HRT, and after only six weeks, merit further research. HRV can be a powerful tool to assess cardiac autonomic function and may be useful in clinical evaluation and management of pathophysiological processes in older postmenopausal women, although age appropriate referent HRV values would facilitate interpretation of findings and delineate age from 
disease effects. This study has posed many additional areas for further investigation, and interpretations of findings are not suggestive of causality. However, this study has provided corroborative support to the previous two studies on hormonal influences in PMW. The extent to which HRT impacts HRV is unclear, but it is worthwhile to continue further investigation into HRV analysis in PMW for its implications on risk stratification and clinical management within this population. Both CVD risk factors of race and HTN appear to be focused areas for additional inquiry with PMW using HRV to assess cardiovascular autonomic function. 
LIST OF REFERENCES 


\section{REFERENCES}

American College of Physicians. (1992). Guidelines for counseling postmenopausal women about preventive hormone therapy. Annals of Internal Medicine, 117, 1038-1041.

American Heart Association. (1998). Risk factors and coronary heart disease. [OnLine]. Available: http://www.amhrt.org/Heart_and_Stroke_A_Z_Guide/riskfact.html.

Barrett-Connor, E. (1995). Postmenopausal estrogen and heart disease. Atherosclerosis, 118, Supplement, S7-S10.

Barrett-Connor, E., Wingard, D. L., \& Criqui, M. H. (1989). Postmenopausal estrogen use and heart disease risk factors in the 1980s. Journal of the American Medical Association, 261, 2095-2100.

Benson, M. D. (1995). Gynecologic pearls: A practical guide for the efficient resident. Philadelphia: Davis.

Bonnet, M. \& Arand, D. (1997). Heart rate variability: Sleep stage, time of night, and arousal influences. Electroencephalography and Clinical Neurophysiology, 102, 390396.

Braune, S., Aver, A., Schulte-Monting, J., Schwerbrock, S., \& Lucking, C. (1996). Cardiovascular parameters: Sensitivity to detect autonomic dysfunction and influence of age and sex in normal subjects. Clinical Autonomic Research, 6, 3-15.

Brown, J. K. \& Kerns, V. (1985). In her prime: A new view of middle-aged women. South Hadley, MA: Bergin \& Garvey.

Burger, H. G., Dudley, E. C., Hopper, J. L., Shelly, J. M., Green, A., Smith, A., Dennerstein, L., \& Morse, C. (1995). The endocrinology of the menopausal transition: A cross-sectional study of a population-based sample. Journal of Clinical Endocrinology Metabolism, 80, 3537-3545. 
Calle, E., Miracle-McMahill, H., Thun, M., \& Heath, C. (1995). Estrogen replacement therapy and risk of fatal colon cancer in a prospective cohort of postmenopausal women. Journal of National Cancer Institute, 87, 517-523.

Campbell, D. T., \& Stanley, J. C. (1963). Experimental and quasi-experimental designs for research. Boston: Houghton Mifflin.

Cashion, A. (1998). Twenty-four hour heart rate variability measures in healthy controls. Unpublished raw data, University of Tennessee, Memphis.

Castelli, W. P. (1994). Menopause and cardiovascular disease. In B. Eskin (Ed.), The menopause: Comprehensive management (3rd ed., pp. 117-136). New York: Field and Wood Medical Publishers.

Chiron/Diagnostic Corporation®. (1996). Ciba-Corning ACS:180 Plus Automated Chemiluminescence System. Medfield:MA.

Chokroverty, S. (1993). Sleep apnea and autonomic failure. In P. Low (Ed.), Clinical autonomic disorders: Evaluation and management (pp. 589-603). Boston: Little, Brown., and Company.

Colditz, G. A., Hankinson, S. E., \& Hunter, D. J. (1995). The use of estrogens and progestins and the risk of breast cancer in postmenopausal women. New England Journal of Medicine, 332, 1589.

Collins, P., \& Beale, C. M. (1996). The cardioprotective role of HRT: A clinical update. New York: Parthenon.

Coney, S. (1994). The medicalization of midlife. In S. Coney (Ed.), The menopause industry: How the medical establishment exploits women (pp. 18-39). Emeryville, CA: Hunter House.

Cowan, M. (1995). Measurement of heart rate variability. Western Journal of Nursing, 17, 32-48. 
Cowan, M. J., Pike, K., \& Burr, R. L. (1994). Effects of gender and age on heart rate variability in healthy individuals and in persons after sudden cardiac arrest. Journal of Electrocardiology, 27 (Supplement), 1-9.

Cowan, M. J., Pike, K., Burr, R. L., Cain, K. C., \& Narayanan, S. B. (1993). Description of time- and frequency- domain-based measures of heart rate variability in individuals taking antiarrhythmics, beta blockers, calcium channel blockers, and/or antihypertensive drugs after sudden cardiac arrest. Journal of Electrocardiology, 26, 1-13.

Davy, K. P. (1996). Elevated heart rate variability in physically active postmenopausal women: A cardioprotective effect? American Journal of Physiology, 27, H455-H460.

Engelhardt, K., Pace, D., Hathaway, D., \& Anderson, R. (November, 1997). Estrogen and cardiac autonomic function in healthy women (Poster presented at the meeting of the American Autonomic Society ). Honolulu, HI

Eskin, B. A. (1994). The menopause and aging. In B. Eskin (Ed.), The menopause: Comprehensive management (3rd ed., pp. 1-26). New York: Field \& Wood Medical Publishers.

Ettinger, B. (1994). Use of low-dosage 17 Beta-Estradiol for the prevention of osteoporosis. Clinical Therapeutics, 16, 950-961.

Ettinger, B., Quesenberry, C., Schroeder, D., \& Friedman, G. (1997). Long-term postmenopausal estrogen therapy may be associated with increased risk of breast cancer: A cohort study. Menopause: The Journal of the North American Menopause Society, 4, 125129.

Flint, M., \& Samil, R. (1990). Cultural and subcultural meanings of the menopause. Anals of the New York Academy of Science, 592, 130-136.

Faulkner, D., Hutchins, D., \& McCollam, J. (1997). Patient compliance with hormone replacement therapy: An analysis of a large prescription claims database (Abstract 
presented at the 8th annual meeting of The North American Menopause Society ). Boston, MA.

Formanek, R. I.. (1990). Continuity and change and "The Changing Life":

Premodern views of the menopause. In K. R. Formanek (Ed.), The meaning of

menopause: Historical, medical, and clinical perspectives (pp. 1-37). Hillside, NJ: Analytic Press.

Gambrell, R. D. (1995). Hormone replacement therapy (4th ed.). Durant, OK:

Essential Medical Information Systems.

Gorodeski, G. I. (1994). Impact of the menopause on the epidemiology and risk factors of coronary artery heart disease in women. Experimental Gerontology, 29, 357375.

Grady, D., Rubin, S. M., Petitti, D. B., Fox, C. S., Black, D., \& Ettinger, B. (1992). Hormone therapy to prevent disease and prolong life in postmenopausal women. Annals of Internal Medicine, 117(1016-1037).

Grady, D., \& Ernster, V. (1991). Invited commentary: Does postmenopausal hormone therapy cause breast cancer? American Journal of Epidemiology, 134, 13961400.

Gupta, S., \& Rymer, J. (1996). Hormone replacement therapy and cardiovascular disease. International Journal of Gynecology \& Obstetrics, 52, 119-125.

Hargrove, J. T., \& Eisenberg, E. (1995). Menopause. Office Gynecology, 89, $1337-1356$.

Hartshorne, C. (1978). The organism according to process philosophy. In S. F. Spicker (Ed.), Organism, medicine, and metaphysics (pp. 137-154). Dordrecht, Holland: D. Reidel.

Howorka, K., Pumpria, J., Haber, P., Koller-Strametz, J., Mondrzyk, J., \& Schabmann, A. (1997). Effects of physical training on heart rate variability in diabetic 
patients with various degrees of cardiovascular autonomic neuropathy. $\underline{\text { Cardiovascular }}$ Research, 34, 206-214.

Huikuri, H. V., Pikkujamsa, S. M., Airaksinen, K. E., Ikaheimo, M. J., Rantala, A. O., Kauma, H., Lilja, M., \& Kesaniema, Y. A. (1996). Sex-related differences in autonomic modulation of heart rate in middle-aged subjects. Circulation, 94(2), 122-125.

Jacox, A. K. (1974). Theory construction in nursing: An overview. In L. H. Nicoll (Ed.), Perspectives on nursing theory (2nd ed., pp. 348-361). Philadelphia: Lippincott . Kageyama,T., Nishikido. N., Honda, Y., Kurokawa, Y., Imai, H., Kobayashi, T., Kaneko, T., \& Kabuto, M. (1997). Effects of obesity, current smoking status, and alcohol consumption on heart rate variability in male white-collar workers. International Archives of Occupational Environmental Health, 69, 447-454.

Kawas, C., Resnick, S., Morrison, A., Brookmeyer, R., Corrada, M., Zonderman, A., Bacal, C., Longle, D., \& Metter, E. (1997). A prospective study of estrogen replacement therapy and the risk of developing Alzheimer's disease. Neurology, $\underline{48}, 1517-1521$.

Keehn, M. (1992). Heart rate variability: Physician's guide. (2nd ed.). Milwaukee, WI: Marquette Electronics.

Kleiger, R. E., Bigger, J. T., Bosner, M. S., Chung, M. K., Cook, J. R., Rointzky, L. M., Steinman, R., \& Fleiss, J. L. (1991). Stability over time of variables measuring heart rate variability in normal subjects. American Journal of Cardiology, 68, 626-630.

Kleiger, R. E., Miller, J. P., Bigger, J. T., \& Moss, A. J. (1987). Decreased heart rate variability and it's association with increased mortality after acute myocardial infarction. Cardiology, 59, 256-262.

Koh, K., Mincemoyer, R., Bui, M., Csako, G., Pucino, F., Guetta, V., Waclawiw, M., \& Cannon, R. (1997). Effects of hormone-replacement therapy on 
fibrinolysis in postmenopausal women. The New England Journal of Medicine, 336, 683690.

Kronenberg, F. (1990). Hot flashes: Epidemiology and physiology. Annals of the New York Academy of Science, 592, 52-86.

Krouse, T. B. (1994). Menopausal pathology. In B. Eskin (Ed.), The menopause: Comprehensive management (3rd ed., pp. 27-70). New York: Field \& Wood Medical Publishers.

Lerman, R. H. (1997). Obesity: An escalating problem: Addressing the genetic and environmental factors. Contemporary Internal Medicine, 9, 14-21.

Levy, W., Cerqueria, M., Harp, G., Abrass, I., Schwartz, R., \& Stratton, J. (1992). Exercise training increases heart rate variability in healthy young and elderly males. Circulation, 86, 588.

Liao, D., Barnes, R., Chambless, L., Simpson, R., Solie, P., \& Heiss, G. (1995). Age, race, and sex differences in autonomic cardiac function measured by spectral analysis of heart rate variability: The ARIC study. The American Journal of Cardiology, $\underline{76}, 906-912$.

Lip, G. Y., Beevers, G., \& Zarifis, J. (1995). Hormone replacement therapy and cardiovascular risk: The cardiovascular physicians' viewpoint. Journal of Internal Medicine, 238, 389-399.

Logothetis, M. (1993). Disease or development: Women's perceptions of menopause and the need for hormone replacement therapy. In J. Callahan (Ed.), Menopause: A midlife passage (pp. 123-135).

Low, P. A. (1993). The effect of aging on the autonomic nervous system. In P. Low (Ed.), Clinical autonomic disorders: Evaluation and management (pp. 685-700). Boston: Little, Brown and Company. 
MacPherson, K. I. (1981). Menopause as disease: The social construction of a metaphor. Advances in Nursing Science, 3, 95-103.

MacPherson, K. I. (1993). The false promises of hormone replacement therapy and current dilemmas. In J. Callahan (Ed.), Menopause: A midlife passage (pp. 145-159).

MacPherson, K. I. (1995). Going to the source: Women reclaim menopause. Feminist Studies, 21 (2), 347-357.

Magid, N. M., Martin, G. J., Kehoe, R. F., Zheutlin, T. A., Meyers, G. A., Eckberg, D. L., Barnett, P. S., Weiss, J. S., Lesch, M., \& Singer, D. H. (1985). Diminished heart rate variability in patients with sudden cardiac death. Circulation, 72, supplement III.

Marquette Electronics, (1990). Heart rate variability: Physician's Guide . Milwaukee, WI: Marquette Electronics.

Martin, G. L., Magid, N. M., Meyers, G., Barnett, P. S., Schaad, J. W., Weiss, J. S., Lesch, M., \& Singer, D. H. (1987). Heart rate variability and sudden death secondary to coronary artery disease during ambulatory electrocardiographic monitoring. The American Journal of Cardiology, 60, 86-89.

Mastrangelo, R. (October, 1993). The menopausal woman: Addressing her concerns effectively. Advance for Nurse Practitioners, 10-26.

Melton, L. J., Thamer, M., Ray, N. F., Chan, J. K., Chesnut, C. H., Einhorn, T. A., Johnston, C., Raisz, L. G., Silverman, S. L., \& Siris, E. S. (1997). Fractures attributable to osteoporosis: Report from the National Osteoporosis foundation. Journal of Bone Mineral Research, 12, 16-23.

Murata, K., Landigran, P. J., \& Araki, S. (1992). Effects of age, heart rate, gender, tobacco and alcohol ingestion on R-R interval variability in human ECG. Journal of Autonomic Nervous System, 37, 199-206. 
Nabulsi, A., Folsom, A. R., White, A., Patsch, W., Heiss, G., Wu, K., \& Szklo, M. (1993). Association of hormone-replacement therapy with various cardiovascular risk factors in postmenopausal women: The Atherosclerosis Risk in Communities Study investigators. New England Journal of Medicine, 328, 1069-1075.

Newman, K. P., \& Sullivan, J. M. (1995). Coronary heart disease in women: Epidemiology, clinical syndromes, and management. Menopause: The Journal of the North American Menopause Society, 3, 51-59.

Notelovitz, M. (1989). Estrogen replacement therapy: Indications, contraindications, and agent selection. American Journal of Obstetrics and Gynecology, $\underline{161}, 1832-1841$.

Omboni, S., Parati, G., Rienzo, M. D., Wieling, W., \& Mancia, G. (1996). Blood pressure and heart rate variability in autonomic disorders: A critical review. $\underline{\text { Clinical }}$ Autonomic Research, 6, 171-182.

Osterhues, H., Hanzel, S. R., Kochs, M., \& Hombach, V. (1997). Influence of physical activity on 24-hour measurements of heart rate variability in patients with coronary artery disease. American Journal of Cardiology, 80, 1434-1437.

Pace, D., Engelhardt, K., \& Hathaway, D. (April, 1997). Autonomic function in patients scheduled for renal/renal-pancreas transplantation: Difference between genders (Oral presentation at Sigma Theta Tau Annual Mid-South Conference for Research in Nursing and Health Care ). Memphis, TN.

Paganini-Hill, A., \& Henderson, V. W. (1994). Estrogen deficiency and risk of Alzheimer's disease in women. American Journal of Epidemiology, 140, 256-261.

Porterfield, S. P. (1997). Endocrine physiology. St. Louis: Mosby.

Ramsay, B., Johnson, M. R., Leone, A. M., \& Steer, P. J. (1995). The effect of exogenous oestrogen on nitric oxide production in women: A placebo controlled crossover study. British Journal of Obstetrics and Gynaecology, 102, 417-419. 
Reardon, M., \& Malik, M. (1996). Changes in heart rate variability with age. Pacing Clinical Electrophysiology, 19, 1863-1866.

Rebar, R. (1994). Unanswered questions in hormonal replacement therapy. Experimental Gerontology, 29(3/4), 447-461.

Richelson, L. S., Wahner, H. W., Melton, L. J., \& Riggs, B. L. (1984). Relative contribution of aging and estrogen deficiency to postmenopausal bone loss. New England Journal of Medicine, 311, 1273-1275.

Rosano, G., Patrizi, R., Leonardo, F., Ponikowski, P., Collins, P., Sarrel, P., \& Chierchia, S. (1997). Effect of estrogen replacement therapy on heart rate variability and heart rate in healthy postmenopausal women. The American Journal of Cardiology, 80, $815-817$

Rosselli, M., Imthurn, B., Keller, P., Jackson, E., \& Dubey, R. (1995). Circulating nitric oxide (nitrite/nitrate) levels in postmenopausal women substituted with 17 B -Estradiol and Norethisterone Acetate. Hypertension, 25, 848-853.

Rothert, M., Rovner, D, Holmes, M., Schmitt, N, Talarczyk, G., Kroll, J., \& Gogate, J. (1990). Women's use of information regarding hormone replacement therapy. Research in Nursing and Health, 13, 355-366.

Rozenberg, S., Liebens, I., Vandromme, J., Hotimsky, A., \& Rijsselberge, M. V. (1994). Cardiovascular protection by estrogen: A hemodynamic mechanism. International Journal of Fertility and Menopausal Study, 39 (Supplement 1), 36-42.

Salo, T. M., Viikari, J. S., Antila, K. J., Voipio-Pulkki, L. M., Jalonen, J. O., \& Valimaki, I. A. (1996). Antihypertensive treatment and heart rate variability in diabetic patients: Role of cardiac autonomic neuropathy. Journal of Autonomic Nervous System, $\underline{60}, 61-70$.

SAS Institute, I. (1995). JMP Statistical Discovery Software (Version 3.1). Cary, NC. 
Saul, J. P., Arai, Y., Berger, R. D., Lilly, L. S., Colucci, W. S., \& Cohen, R. J. (1988). Assessment of autonomic regulation in chronic congestive heart failure by heart rate spectral analysis. The American Journal of Cardiology, 61, 1292-1298.

Scharbo-Dehaan, M. (1996). Hormone replacement therapy. The Nurse Practitioner: The American Journal of Primary Health Care, 21(Part 2 of 2), 1-13.

Schinfeld, J. S. (1994). Sex steroid hormone metabolism in the climacteric woman. In B. E. (Ed.) (Ed.), The menopause: Comprehensive management (3rd. ed., pp. 269288). New York: Field and Wood Medical Publishers, Inc.

Schwartz, J. B., Gibb, W. J., \& Tran, T. (1991). Aging effects on heart rate variation. Journal of Gerontology, 46, M99-M106.

Spencer, R., Nichols, L., Lipkin, G., Henderson, H., \& West, F. (1993). Clinical pharmacology and nursing management. (4th ed.). Philadelphia: Lippincott.

Speroff, L., Glass, R. H., \& Kase, N. G. (1994). Clinical gynecologic endocrinology and infertility. (5th. ed.). Baltimore: Williams \& Wilkins.

Stampfer, M. J., \& Golditz, G. A. (1991). Estrogen replacement therapy and coronary disease: A quantitative assessment of the epidemiological evidence. Preventive Medicine, 20, 47-63.

Steinberg, K., Smith, S. J., Thacker, S. B., \& Strough, D. F. (1994). Breast cancer risk and duration of estrogen use: The role of study design in meta-analysis. Epidemiology, 5, 415-421.

Steinberg, K., Thacker, S. B., \& Smith, J. (1995). A meta-analysis of the effect of estrogen replacement therapy on the risk of breast cancer. Journal of the American Medical Association, 1991, 1985-1990.

Stenberg, A., Heimer, G., \& Ulmsten, U. (1995). The prevalence of urogenital symptoms in postmenopausal women. Maturitas, 22 (Supplement), S17-S20. 
Sudhir, K., Jennings, G. L., Funder, J. W., \& Komesaroff, P. A. (1996).

Estrogen enhances basal nitric oxide release in the forearm vasculature in perimenopausal women. Hypertension, 28, 330-334.

Tiller, W. A., McCraty, R., \& Atkinson, M. (1996). Cardiac coherence: A new noninvasive measure of autonomic nervous system order. Alternative Therapies in Health and Medicine, 2(1), 52-65.

Tsuji, H., Larson, M. G., Venditti, F. J., Manders, E. S., Evans, J. C., Feldman, C. L., \& Levy, D. (1996a). Impact of reduced heart rate variability on risk for cardiac events: The Framingham heart study. Circulation, 94, 2850-2855.

Tsuji, H., Venditti, F. J., Manders, E. S., Evans, J. C., Larson, M. G., Feldman, C. L., \& Levy, D. (1996b). Determinants of heart rate variability. Journal of American College of Cardiology, 28, 1539-1546.

Utian, W. H. (1990). The menopause in perspective: From potions to patches. In M. Flint, F. Kronenberg, \& W. Utian (Eds.), Multidisciplinary perspectives on menopause (Vol. 592, pp. 1-7). New York: New York Academy of Sciences.

Utian, W. H., \& Schiff, I. (1994). NAMS-Gallup survey on women's knowledge, information sources, and attitudes to menopause and hormone replacement therapy. Menopause: The Journal of the North American Menopause Society, 1, 39-48.

van Ravenswaaij-Arts, C., Kollee, L. A., Hopman, J. C., Stoelinga, G. B., \& van Geijn, H. P. (1993). Heart rate variability. Annals of Internal Medicine, 118, 436-447.

Veerman, D. P., Imholz, B., Wieling, W., Karemaker, J. M., \& van Montfrans, G. A. (1994). Effects of aging on blood pressure variability in resting conditions. Hypertension, 24, 120-130.

Voda, A. (1993). A journey to the center of the cell: Understanding the physiology and endocrinology of menopause. In J. Callahan (Ed.), Menopause: A midlife passage (pp. 160-193). Bloomington, IN: Indiana University Press. 
Voda, A. M. (1992). Menopause: A normal view. $\underline{\text { Clinical Obstetrics and }}$ Gynecology, 35, 923-933.

Wellman, G. C., Bonev, A. D., Nelson, M. T., \& Brayden, J. E. (1996). Gender differences in coronary artery diameter involve estrogen, nitric oxide, and $\mathrm{Ca}(2+)$ dependent K+ channels. Circulation Research, 79, 1024-1030.

Wise, P., Kranjnak, K., \& Kashon, M. (1996). Menopause: The aging of multiple pacemakers. Science, 273, 67-70.

World Health Organization Scientific Group on Research on the Menopause in the 1990’s. (1996). WHO technical report series (pp. 886). Geneva, Switzerland.

Writing Group for the PEPI Trial. (1996). Effects of hormone therapy on bone mineral density: Results from the postmenopausal estrogen/progestin (PEPI) trial. Journal of the American Medical Association, 276, 1397-1403.

Wyeth-Ayerst Laboratories. (1997, January). Information for the patient (Publication No. 38383-00). Philadelphia, PA.

Wyeth-Ayerst Laboratories. (1997, April). What to expect from your therapy with $\underline{\text { PremPro }}{ }^{\text {TM }}$ (Publication No. PI4665-2). Philadelphia, PA.

Zita, J. N. (1993). Heresy in the female body: The rhetorics of menopause. In J. C. Callahan (Ed.), Menopause: A midlife passage (pp. 59-78). Bloomington, IN: Indiana University Press. 
APPENDICES 
Appendix A

Approval Letters 
THE UNIVERSITY OF TENNESSEE

MEMPHIS

The Health Science Center

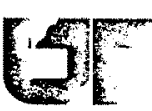

Institutional Review Board

62 South Dunlap, Suite 320

Memphis, TN 38163

(901) $448-4824$

September 4, 1997

Diane T. Pace

College of Nursing

620 Alexander Building

UT Memphis

Re: IRB \#6339 - "The Effect of Hormone Replacement Therapy in Postmenopausal Women on Cardiac Autonomic Function"

Dear Ms. Pace:

We are in receipt of your written acceptance of the provisos outlined in my letter of August 20, 1997, concerning the above referenced Institutional Review Board protocol. We have reviewed these materials and find that they do comply with the proper consideration for the rights and welfare of human subjects, the risk involved, and the potential benefits of the study. Therefore, this letter constitutes full approval from the Institutional Review Board for the above referenced study and consent form.

However, any further alterations in the protocol must be promptly reported to and approved by the UT Memphis Institutional Review Board (IRB). In addition, annual reapproval is required by the IRB, and it is the responsibility of the Principal Investigator to initiate the request for reapproval regardless of the time the activity has been approved by the sponsoring agency.

You have individual responsibility for reporting to the Board in the event of adverse reactions.

Sincerely,

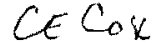

Clair E. Cox, M.D.

Chairman

Institutional Review Board 


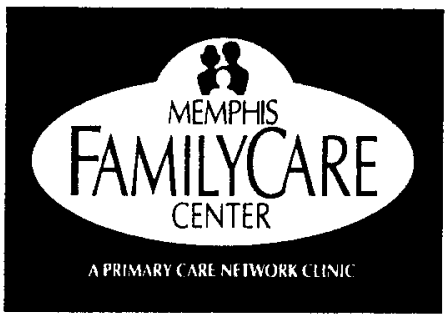

August 25, 1997

Diane Pace, $\mathrm{PhD}(\mathrm{c}), \mathrm{RN}, \mathrm{CS}$

Family Nurse Practitioner

8185 Timber Hill Trail

Cordova, TN 38018

Dear Diane:

This letter is to approve your request to recruit patients for participation in and conduct cardiac autonomic function testing at the Memphis FamilyCare Centers in partial fulfillment of your doctoral research project, "The Effect of Hormone Replacement Therapy in PostMenopausal Women on Cardiac Autonomic Function". I understand that this project has been reviewed and approved by the University of Tennessee, Memphis, Institutional Review Board (IRB) and found to comply with proper consideration of the rights and welfare of human subjects, the risk involved, and the potential benefits of the study. A copy of the IRB approval as well as a copy of the Consent Form used for this study are on file in our corporate office.

Sincerely,

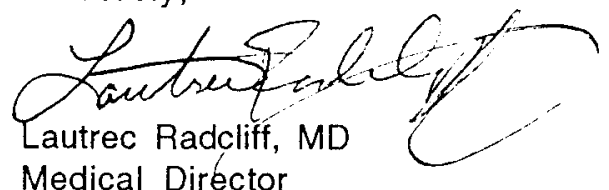

Medical Director 
WYETH-AYERST PHARMACEUTICALS

Pbiladelphia, PA 19101-8299 • (610) 902-1200

Division of American Home Products Corporation

$1-98$

Wyeth-Ayerst Laborotories

Mike Musick

3447 Angelin

Bartlett,Tn.38135

\section{Dear Sirs,}

We agree to supply the medical providers with Prempro samples for a study. We understand that these samples will be distributed to patients as needed.

Thank You,

mine Musuch

Mike Musick

\section{Wyeth}

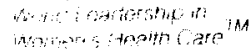

MIKE MUSICK

TERRITORY SPECIALIST 


\section{Appendix B}

Prescriptive Authority for Principal Investigator 


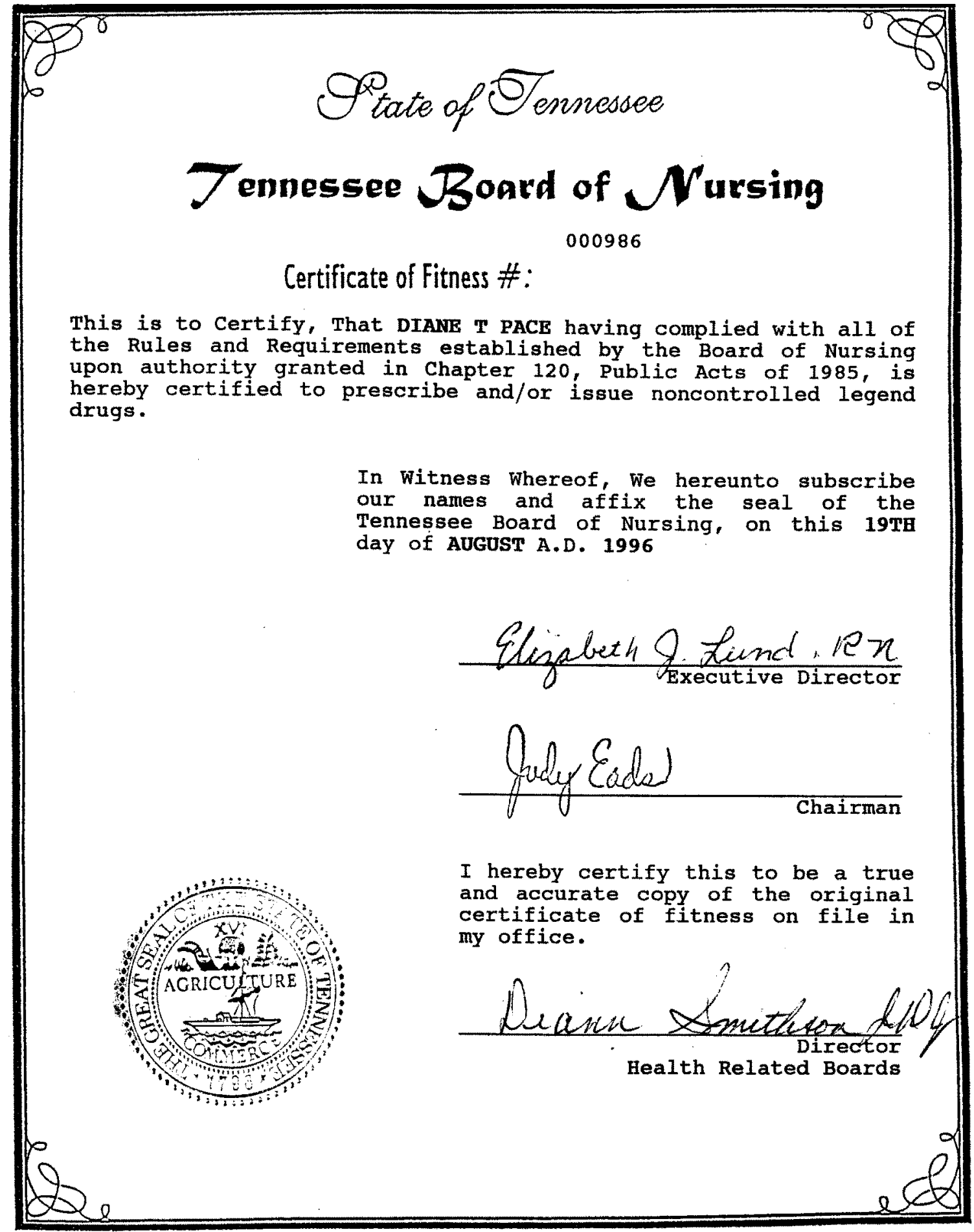


Appendix C

Study Instruments 
PRE/ POST-TEST HEALTH FORM

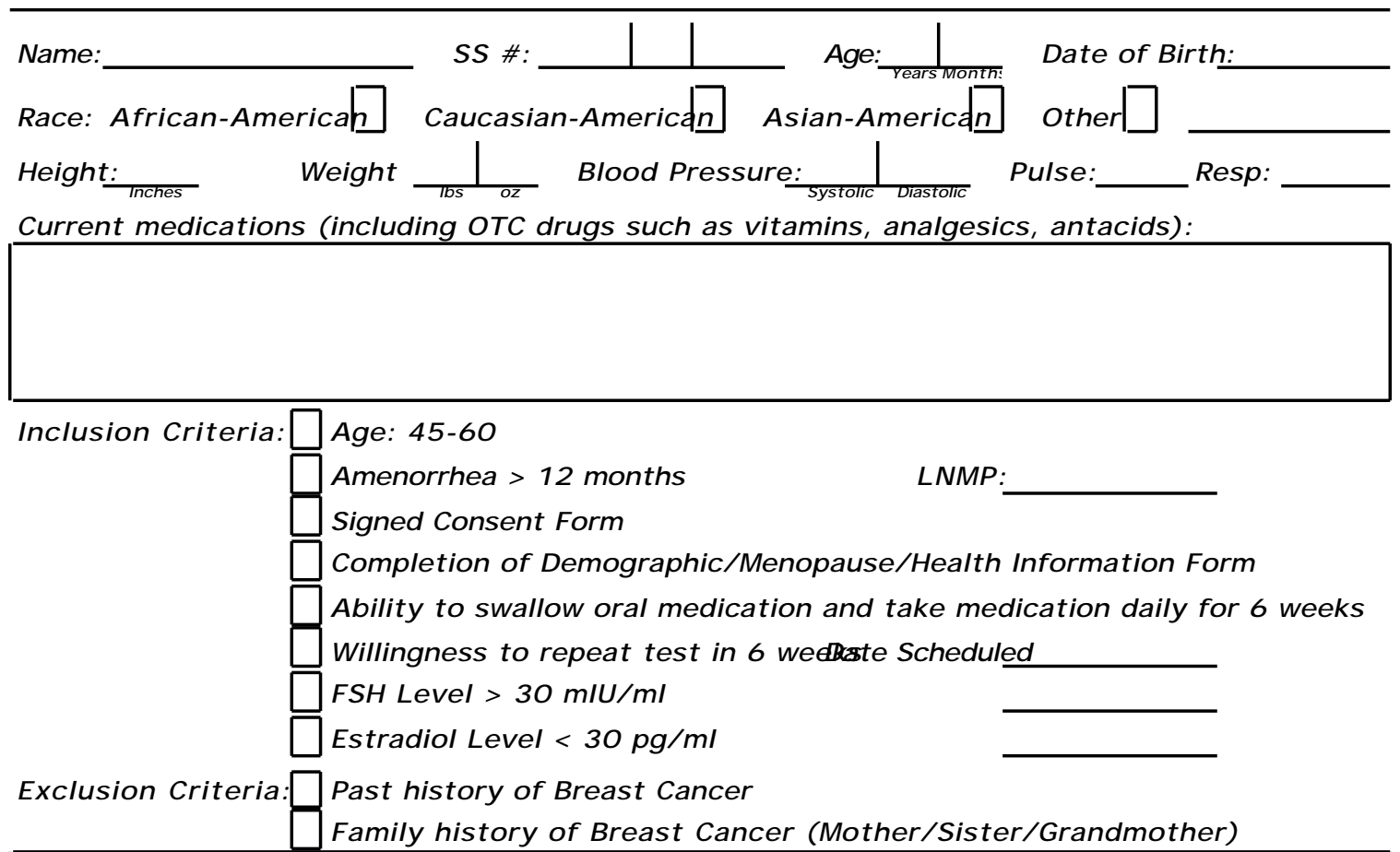

Name/ Phone \# of 3 relatives or friends who can be contacted in the event subject's phone number changes:

Heart Rate Variability AnalysiÆrequency Domains:Total Hertz

Low Hertz

High Hertz

Low/ High Ratio

Time Domains: $\quad$ pNN50

SD

SDNN

SDANN

Nitric Oxide:

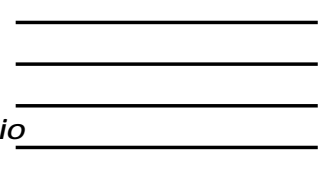

\begin{tabular}{|ll|l|}
\hline \hline Research Nurse/Screener & Date of Test & Pre-treatment \\
\hline Principal Investigator & Date of Analysis & Referral Site: \\
\hline Additional Comments: & & Subject Phone No: \\
\hline
\end{tabular}




\section{GENERAL INFORMATION}

Please complete the following personal information. All information will be collected and reported as a group No identifying individual information will be used.

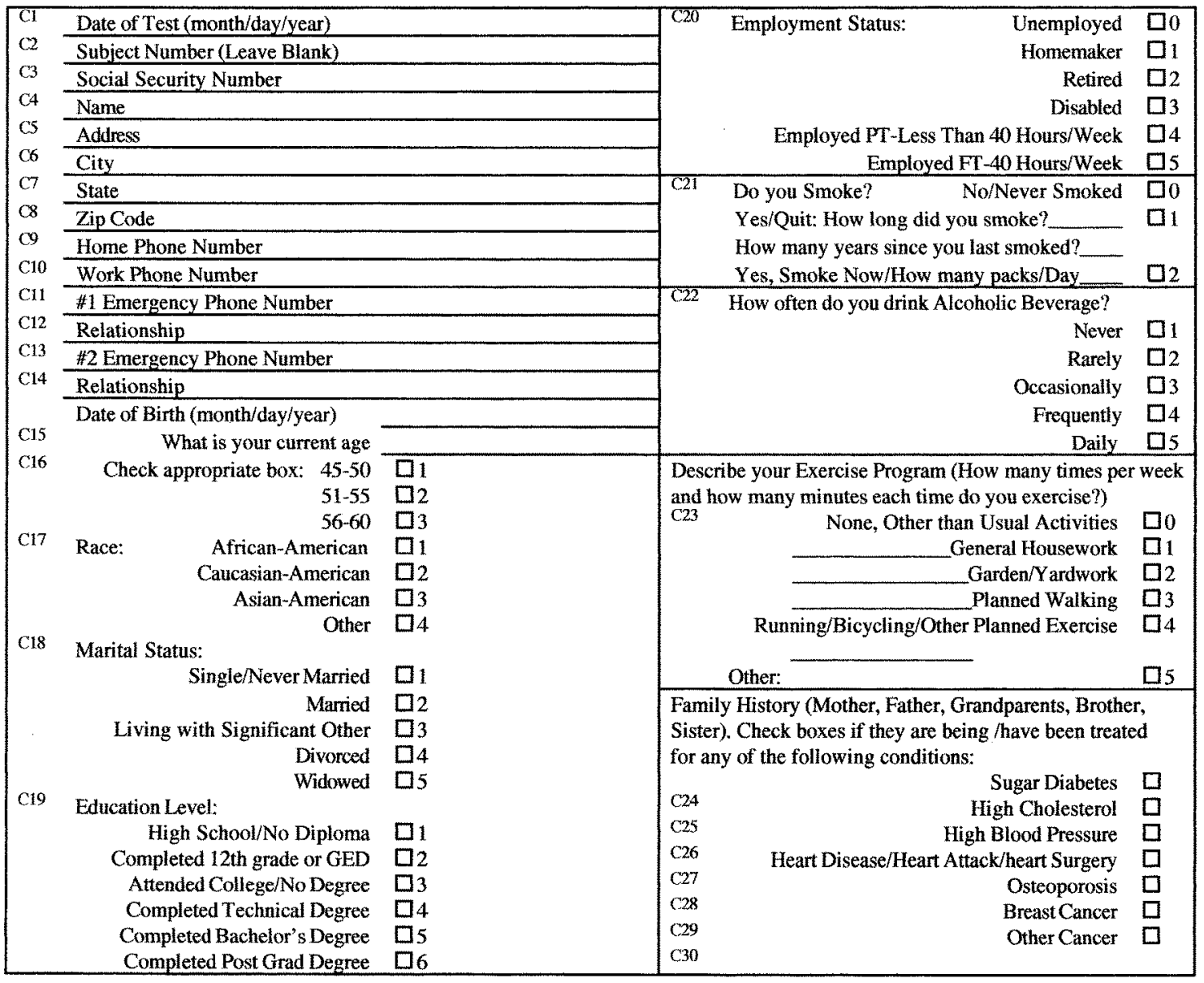

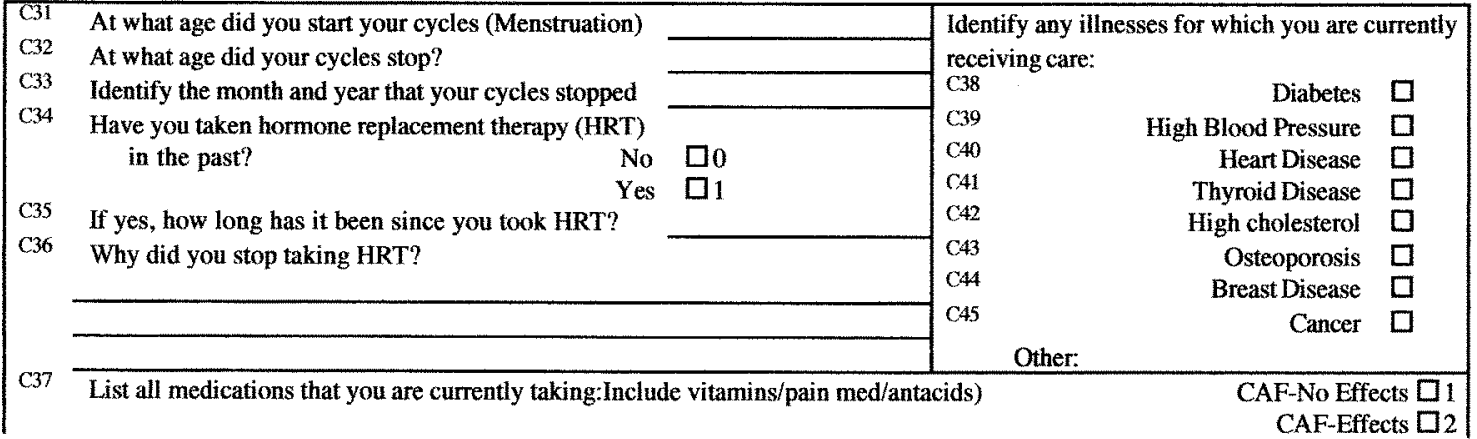


Appendix D

Protocols for Holter Monitoring 
PROTOCOL \#: $\underline{07}$

PAGE $\underline{1}$ OF $\underline{1}$

\section{HOLTER MONITORING: \\ PATIENT PREPARATION/ ELECTRODE PLACEMENT}

1. To minimize electrode problems, ensure good quality by checking for expiration date. If patient is to only have Holter Monitoring, use 5 electrodes. If Autonomic Function is also is to be done, use 7 electrodes.

2. Apply shaving lotion and using safety razor, shave individual sites. Shaving improves conductivity, holds electrode to the skin, and facilitates removal of electrode.

3. If skin is extremely oily, first wash designated area with soap and water. Clean site with alcohol swab. Allow to air dry or dry with clean $4 \times 45$.

4. Apply "dot" of liquid sandpaper, rubbing into skin with $4 \times 4 \mathrm{~s}$, or use sandpaper squares to further prepare each individual electrode site.

5. If patient is to only receive Holter testing, connect lead wires to each electrode prior to applying to patient. If patient is to have Autonomic Function testing completed first, do not attach leads prior to electrode placement.

6. Apply electrodes. Tape each lead wire to the chest using a stress loop to prevent the wires from pulling directly on the electrode contact points. The loops should be approximately 1 to 2 inches in diameter, about 2 inches from the electrodes.

\begin{tabular}{|c|lc|}
\hline \multicolumn{3}{|c|}{ ELECTRODE PLACEMENT } \\
\hline LEAD COLOR & \multicolumn{3}{|c|}{ LOCATION } \\
\hline White & Mid-Sternum-Over Manubrium & 1.5 inches from cla \\
\hline Green & Right Mid-Clavicular Line & Below Costal Marg \\
\hline Red & Left Mid-Clavicular Line & Below Costal Marg \\
\hline Brown & Right Mid-Axillary Line & Below Costal Margin \\
\hline Black & Left Mid-Axillary Line & Below Costal Margin \\
\hline
\end{tabular}


PROTOCOL \#: $\underline{08}$

PAGE $\underline{1}$ of $\underline{2}$

\section{HOLTER MONITORING: RECORDER PREPARATION}

1. Use anewcassette tape for each patient. A plastic bar inserted into the tape reels signifies no previous use. Remove the bar from the tape. Using a pencil in the tape reel, wind the tape forward until the blank, white pre-tape is beyond the recording site. This procedure will ensure that data is recorded from the onset of tape recording initiation.

2. Document patient identification information on the cassette label.

Patient's Name

Patient's Social Security Number

Test Date

Time Test was Initiated

Recorder Identification Number: This number is written on the outer metal case.

Patient Category: Pre-Kidney; J uvenile; Women's Study

3. Obtain a new 9-volt alkaline battery.

4. Remove outer metal case of the recorder. Hold the top of the recorder (the end which protrudes beyond the metal case) in one hand. Slide the metal case off with the other hand.

5. Install the battery by inserting it into the battery compartment with the positive $(+)$ and negative $(-)$ terminals aligned according to the diagram inside the compartment. The battery should fit securely in the compartment with no space between the bottom of the battery and the bottom of the compartment.

6. Slide the cassette release to the left. The recording head assembly moves to the OUT position.

7. Place the tape in the recorder with the full reel on the supply (left) hub. Insert the cassette into the recorder by placing the top edge of the cassette in first under the retaining guides and then carefully easing the cassette down over the supply and take-up hubs and the cassette locator pins.

8. Prepare the patient and connect the lead wires.

9. Activate the recorder by pushing the recording head aslstertifbuss $h$ with the side of the recorder). Visualize the take-up hub or the tape reels turning slowly. Leave the case open while completing the additional documentation to ensure recorder is working properly. 
10. Complete the patient documentation on the diary:

Patient Name

Patient Social Security Number

Age Sex

Patient's Medications

Date \& Time test is initiated

Serial No: (This number is the Holter Monitor number

Initials of individual completing test in the space for technician

11. Replace the recorder in the metal case and place inside the canvas purse. Place the Velcro tab across the cable/ recorder site to prevent it from becoming dislodged. Do not push the inset button as it will erase tape.

12. Patient Instructions:

A. Maintain a 24 hour diary of activities. Especially note:

Time went to sleep-including naps

Time awoke

Remembrance of any periods of dreaming while asleep

Exercise

Home Blood Glucose Monitoring Levels $<50$ or $>200$

Time On/ Off Dialysis

Stressful Experiences

B. Do not open the canvas purse or attempt to remove the case, even when the test is completed.

C. Wear the canvas purse by the shoulder strap or attached to a belt.

D. When the 24 hours of testing have been completed, carefully remove the tape over the lead wires and unsnap them from the electrodes. Place the leadwires, cable, and canvas purse in the white envelope for mailing or return the equipment to the admissions office on first floor of Bowld Hospital. Do not attempt to open the case. Remove the electrodes and discard.

13. If patient wishes to mail-in equipment, provide them with a white envelope and a Fed Ex mailing label (See example). Identify the initials of the principle investigator for test on the form. Maintain the "Sender's Copy" in the Physiology Function Laboratory. Document the monitor number and test date on the Sender's Copy for future tracking purposes if necessary.

14. Document patient information into Daily Log Book and on Bulletin Board: Name

Date of Test

Patient's Category: Pre-Kidney J uvenile Women's Study Holter Number Time Holter applied

How Holter will be returned: Patient in town or per Federal Express. 


\section{PATIENT ACTIVITY DIARY}

Record all of your activities during the next 24 hour period on this diary.

Especially note the following infomation: 1) Time you start and stop dialysis. 2) Time you go to sleep, including naps, and the time you wake up. 3) Time that you eat meals. 4) Results of your

Blood Sugar monitoring. 5) Time and type of exercise. 6) Any unusual stress or symptoms.

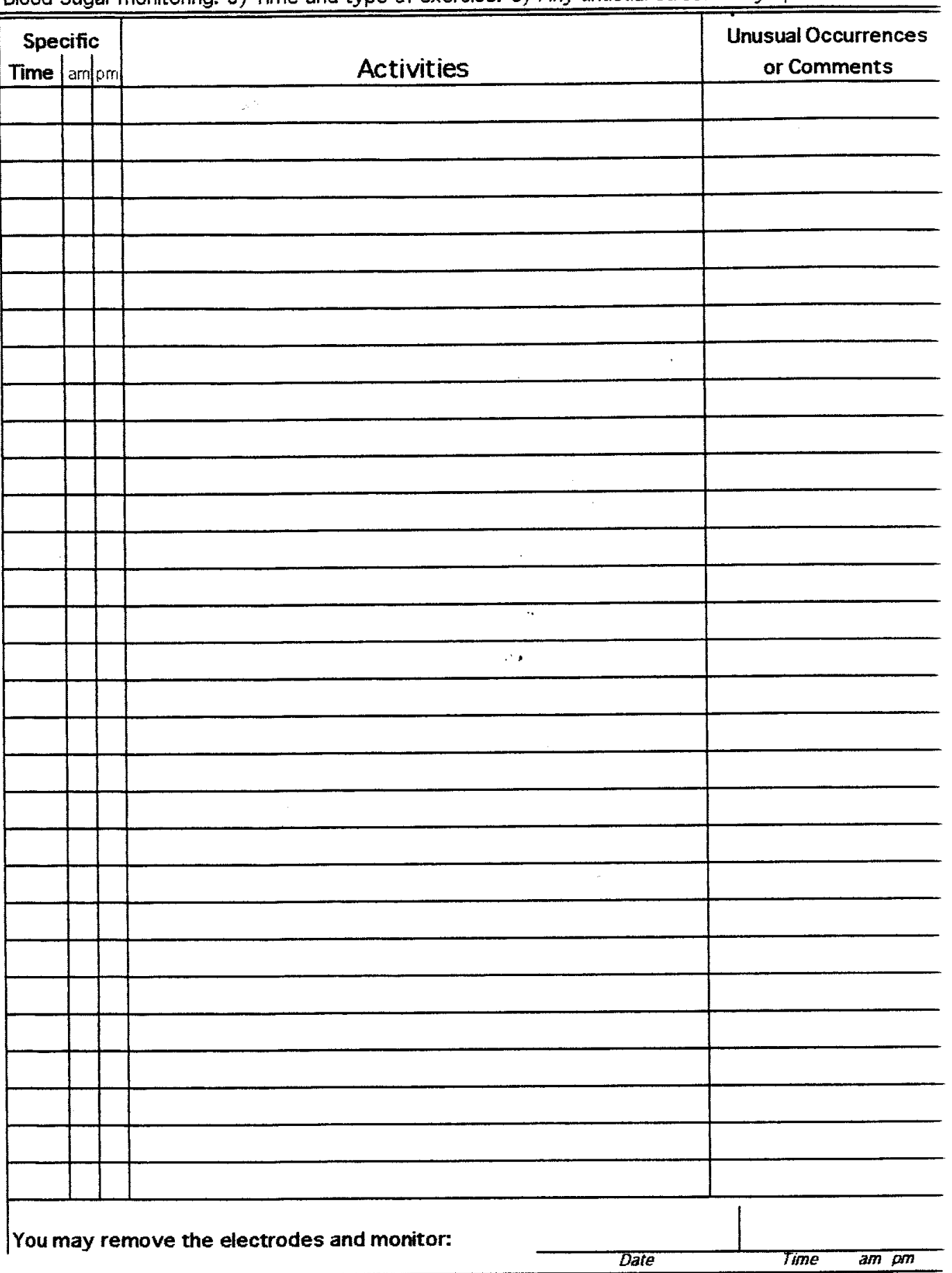


What is this test?

The 24 hour holter monitor is a tape recording of your heart beat. It is recorded on a cassette tape which will later be put on a written report then analyzed.

Record all of your activities on the diary. Especially note the following information:

1 Time you start and stop dialysis.

2 Time you go to sleep, including naps, and the time you wake up.

3 Time of eating meals.

4 Blood sugar monitoring results.

5 Time and type of exercise.

6 Any unusual stress or activities.
What can I do while wearing the Holter recorder?

Follow your normal daily routine, including

eating, working, playing, sleeping!

also follow these instructions:

1. Do not take a bath, shower, swim or get the recorder or electrodes wet.

2. Do not open or tamper with the recorder or disturb the lead wires and/or electrodes.

3. If the electrodes become loose, tape them back on and make a note in your diary.

4. Do not stop the test before the time listed on the diary chart.

Early removal will effect test results.

If you are unable to remove the wires at the exact time, Do Not Worry.

Choose a more later time which will be more convenient. It will not effect the test.

4. Do not use an electric blanket while testing.

At the end of the test, return the monitor,

diary, and any belts to Bowld Hospital

Admissions Office or mail the equipment

and diary as instructed. As a courtesy to our

patients, please return the monitor as soon

as possible.

\section{Director:}

Donna Hathaway, PhD Bowld Hospital

951 Court/ Room $341 \mathrm{M}$

Memphis, TN 38104

901-448-1584
Physiological Function Laboratory Heart Rate Variability Patient Diary

Name:

SS \#:

Age:

Medicines:

Tone

Doctor:

Testing Date:

Time Test Started:

Date and Time Test May Be Stopped:

Study:
Asthma Inhale Beta Blocker Inderal, Atenolol, Lopre Tenorim, Corgard

Ca Channel Blocker

Cardizem, Procardia, Verapa Cyclosporin

Depakote

Digitalis

Dilantin

Diuretic

\begin{tabular}{|l} 
PreTransplant \\
PostTransplant \\
Pediatrics \\
Women's Study \\
Healthy Subject
\end{tabular}

No:

No:

Healthy Subject

Race:

ERT/HRT

Insulin

Hypogly/Oral

Pronestyl

Quinidine

Thyroid

Vasodilator

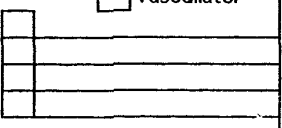

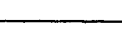

echnician: 
Appendix E

Consent Form 


\section{Consent to Participate in the Study: "Effect of PostMenopausal Hormone Replacement Therapy on Cardiac Autonomic Function"}

\section{Please read each page carefully and place your initials at the bottom of each page where indicated. Assistance with having the form read to you will be provided if needed.}

\section{A. PURPOSE OF STUDY}

Many research studies have shown that low estrogen production in menopause is a risk for heart disease. These studies suggest that hormone replacement therapy (HRT) consisting of estrogen and progesterone, taken by mouth as a pill, may reduce the chances of developing heart disease, especially in postmenopausal women at high risk for heart disease. This study is being done to study the effect of HRT on the cardiac autonomic system. This system is responsible for the heart functions which occur automatically in the body, such as regulating your heart rate and maintaining your blood pressure. By examining these responses, how they change during menopause, and in response to HRT, we might be able to predict which individuals are at risk for heart disease and possibly sudden death.

To complete this study, enrollment of fifty (50) women are necessary. If you agree to participate, you will undergo special testing two (2) times during your six (6) weeks of participation. You may choose to participate in either of two (2) levels of tests. To be included, you must be 12 months past your last menstrual cycle and your blood hormone levels must confirm that you are post-menopausal. You should have no history of breast cancer in either yourself or your grandmother, mother, sister. You must be willing to take two (2) pills daily by mouth for 6 weeks.

\section{B. TESTING PROCEDURES}

\section{QUESTIONNAIRES/HEALTH SCREENING}

The following information will be obtained prior to the tests:

1. Personal information such as your age, race, education, marital status, etc., and health information about your physical/mental health, any medications that you are taking, menopause experience, dietary and exercise habits, etc.

2. Basic physical screening including: height, weight, blood pressure. You also will have $20 \mathrm{ml}$ (approximately 4 teaspoons) of blood drawn to determine your hormone levels and blood nitric oxide level. Nitric oxide helps to dilate your blood vessels and may have a role in influencing cardiac autonomic function. To obtain an accurate result, blood samples must be drawn prior to eating breakfast. Do not eat anything after midnight the night before your test. If you are diabetic or require scheduled meals, bring something to eat to the testing site.

Initials: 


\section{CARDIAC AUTONOMIC TESTS}

You may choose between two types of testing. The location for obtaining testing are different for the two tests. The second set of tests must be done at UT Bowld Research Center at the Medical Center. Please initial to which test you are giving your consent:

1. Holter Monitoring: This test is similar to a cardiogram, except it is done for a full 24 hours rather than in a few minutes. The monitor is similar to a tape recorder, and it constantly records your heart beat on a tape cassette. For this test, we will put 5 patches on your chest and tape them in place. Wires will connect the patches to the monitor. There is no danger from these wires; no electricity is connected to the monitor. However, you will not be able to get the patches wet or they will come off, and the test will not be able to be used. You will wear the monitor by a strap connected to a small purse. This procedure will be explained to you by the research assistant at the time of testing.

2. Cardiac Autonomic Tests: In addition to the Holter monitoring described above, you will have further testing of cardiac autonomic function. These tests must be completed at the Physiologic Function Lab located on 3rd floor of UT Bowld Hospital. The procedures for these tests are:

a. Blood Vessel Test: A small flat metal probe will be taped to the outside of your middle finger and attached to a large machine. This probe measures the blood flow in your finger by using infrared light waves. No needles are used in this test. You will sit in a comfortable chair while the probe is on your finger. We will ask you to complete several tasks during these tests. First, we will record the blood flow in your finger while you sit quietly. Second, we will ask you to raise your arm while we record the blood flow. Third, we will ask you to place your hand in a basin of ice water for one minute, and we will take recordings before and after.

b. Blood Pressure Tests: For these tests you will have a blood pressure cuff put on your arm and attached to a machine that automatically records your blood pressure. You will also have cardiogram patches taped to your chest to record your heart beats. We will ask you to complete several tasks during these tests. First, we will ask you to rest quietly on an exam bed while we record several blood pressures. After we tell you, the bed will be tilted so that you will be placed in a standing position. We will again check your blood pressure before returning the bed to a lying position. Another test will be done in a comfortable chair. We will record your heart beat and blood pressure while you are sitting quietly. Then we will ask you to blow forcefully into a small machine for 15 seconds while we monitor your heart beats.

Initials: 


\section{DRUG INFORMATION}

Because the purpose of this study is to investigate the effect of Hormone Replacement Therapy (HRT) on cardiac autonomic function, you will receive HRT for 6 weeks. The medications you will be receiving are ones which are usually prescribed as hormone replacement for menopausal women. They are not experimental drugs. The medication will be provided for you in packages containing two (2) pills which you will take every day for six (6) weeks. One pill is conjugated estrogen, known as "Premarin", $0.625 \mathrm{mg}$; the other pill is medroxyprogesterone, know as "Provera", $2.5 \mathrm{mg}$. The information sheets on these medications will list potential side effects such as: Headache, dizziness, swelling in your legs, nausea, weight change, breast tenderness. It has been suggested that taking estrogen long term may increase your chance of developing breast cancer. The drug, Provera, is necessary in women who have their uterus (womb) to prevent the risk of endometrial cancer. It will be very important that you take both drugs during the study. The risk of any of the more serious side effects are minimal for this study because of the short time frame (6 weeks) that you will take the drugs.

Also, because of these potential side effects, it is strongly suggested that you notify your healthcare provider (physician or nurse practitioner) of your participation in this study and that you continue your routine health or follow-up visits.

\section{RISKS}

The main risk in this study is exposure to potential side effects of the drugs as previously explained. The testing by the Holter monitor should cause no discomfort other than possible skin irritation at the site where the chest patches are taped. Mild discomfort, bruising, or possible infection at the injection site are potential side effects of having your blood drawn. Additionally, if you choose to participate in the complete cardiac autonomic function tests, mild discomfort may be experienced during the ice test.

\section{LEGAL RIGHTS}

You are a volunteer, and you have the right to stop your participation in this study at any time. You are not releasing any legal rights by your signature, nor are you releasing the university or its agents from any liability or negligence. However your signature on this consent form means that you understand that in the event of physical injury resulting from the research procedures, the University of Tennessee, Memphis does not have funds budgeted for compensation, either for lost wages or for medical treatment. Therefore, the University does not provide for treatment or reimbursement for such injuries.

\section{E. WHO TO CONTACT IF YOU HAVE QUESTIONS}

If you have questions about the study or your rights as a participant in this study, you may contact: Diane Pace, $\mathrm{PhD}(\mathrm{c}), \mathrm{RN}, \mathrm{CS}$, FNP at The University of Tennessee, Memphis at (901) 448-1584. If you have any questions about your medical condition, please contact your healthcare provider. If you have any questions regarding your rights as a research subject, you can contact Dr. Clair Cox, Chairman, University of Tennessee, Memphis, Institutional Review Board at 901-448-4824.

Initials: 


\section{F. BENEFITS OF THIS RESEARCH}

Knowing how HRT effects the changes in heart beat and other cardiac autonomic functions will provide new knowledge on how the drugs benefit women in reducing their risk for heart disease which increases after menopause. By participating in this study you will benefit from having a thorough examination of your autonomic function. You will also help to define the values which might identify risks for cardiac disease.

\section{G. CONFIDENTIALITY}

All information given by you will be kept in strict confidence. Your personal information will be coded with a subject number; no linkage will be made to your name, nor revealed or released during the study, nor in any publication that may be completed following the study completion. If the study results suggest a possible health problem which might require medical attention, you will be notified and asked if you want this information released to your provider.

\section{H. VOLUNTARY CONSENT}

I have read the description of the study entitled: "Effect of PostMenopausal Hormone Replacement Therapy on Cardiac Autonomic Function". I have freely volunteered to participate in it. I have had possible side effects and adverse reactions explained, and I have received a copy of the information sheet for the two drugs, Premarin and Provera. It has been strongly recommended to me that I should alert my healthcare provider of my participation in the study, and that I should continue all follow-up medical visits and/or keep my appointment for my annual exam. I have had the opportunity to ask questions and have received adequate answers. I understand that I may withdraw from this study at any time.

Patient Signature :

Date:

Witness Signature :

Date :

Investigator Signature:

Date : 
Appendix $\mathrm{F}$

Drug Documentation Calendar 


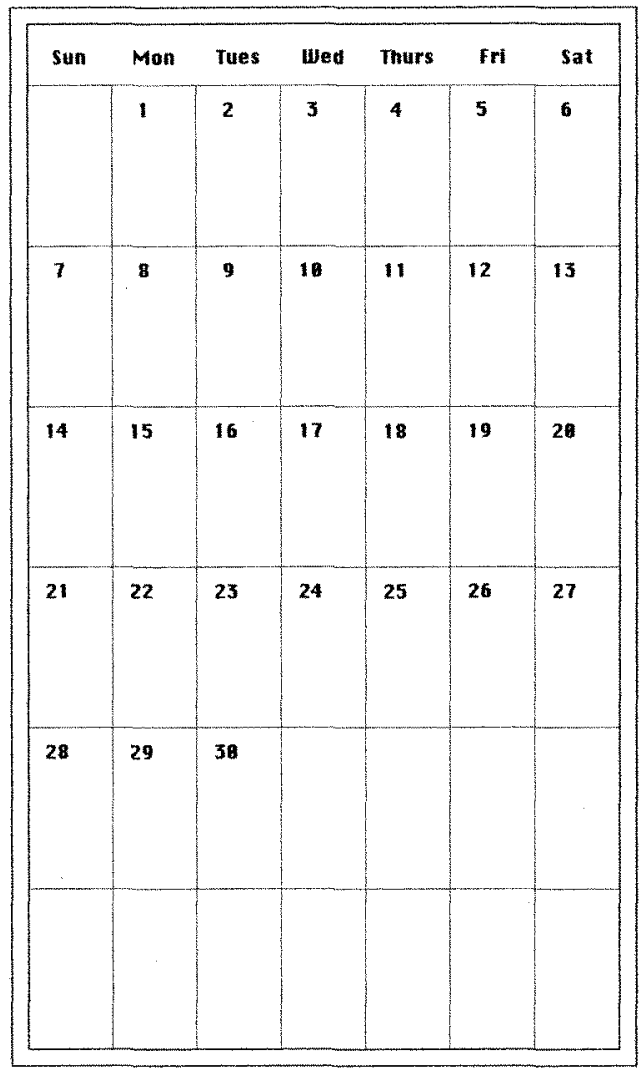

\section{DRUG DOCUMENTATION CALENDAR}

Name:

Mark an " $X$ " on each day that you take your pill. Circle any day that you miss taking a pill.

The following information should be written in the appropriate box if it occurs:

If you visit your healthcare provider and he/she changes your medication.

If you change your usual diet habits.

If you do any exercise, including housework or walking at your job.

If you quit smoking or start smoking.

If you have any vaginal bleeding or spotting.

If you have any unusual symptoms.

\section{September 1997}


Appendix G

Normative HRV Data for Healthy Controls 
Table 15

Heart Rate Variability: Mean, Normal, Borderline, and Abnormal Values Based on the 2.3 and 5th Percentile Value for a Group of Healthy Individuals Tested Through the Physiologic Function Laboratory ${ }^{1}$.

\begin{tabular}{lcccc}
\hline HRV Values & $\begin{array}{c}\text { Mean/SD } \\
\text { Values }\end{array}$ & Normal & Borderline & Abnormal \\
\hline Frequency Domains & & & & \\
Total Hz & $7.25 \pm 0.63$ & $\geq 6.11$ & $5.95-6.10$ & $\leq 5.94$ \\
Low Hz & $6.42 \pm 0.66$ & $\geq 5.22$ & $4.97-5.21$ & $\leq 4.96$ \\
High Hz & $5.2 \pm 0.91$ & $\geq 3.45$ & $3.29-3.44$ & $\leq 3.28$ \\
Low/High Ratio & & & & \\
& & & & \\
Time Domains & $11.03 \pm 8.26$ & $\geq 0.6$ & $0.4-0.5$ & $\leq 0.3$ \\
pNN50 & $58 \pm 15$ & $\geq 33$ & $31-32$ & $\leq 30$ \\
SD & $134 \pm 33$ & $\geq 85$ & $79-84$ & $\leq 78$ \\
SDNN & $121 \pm 32$ & $\geq 69$ & $66-68$ & $\leq 65$ \\
SDANN & $32.8 \pm 13$ & $\geq 14$ & 13 & $\leq 12$ \\
rMSSD & & & & \\
\hline
\end{tabular}

Note. Sample N=158, mean age $=36.2 \pm 11.2$, range: $18-65$ years; $M e n=40(25 \%)$, Women=118 (75\%); Blacks=29 (19\%); Whites=121 (77\%); Other=7 (4\%).

Source. Cashion, A. (1998). Twenty-four hour heart rate variability measures in healthy controls. Unpublished raw data, University of Tennessee, Memphis. 


\section{Appendix $\mathrm{H}$}

Data Tables: Pre-Test Heart Rate Variability for

Postmenopausal Women Across Selected Cardiovascular Risk Factors 
Table 16

Comparisons of Pre-Test Mean Values for Heart Rate Variability for

Postmenopausal Women Differing Across Demographic Variables of Race and Age

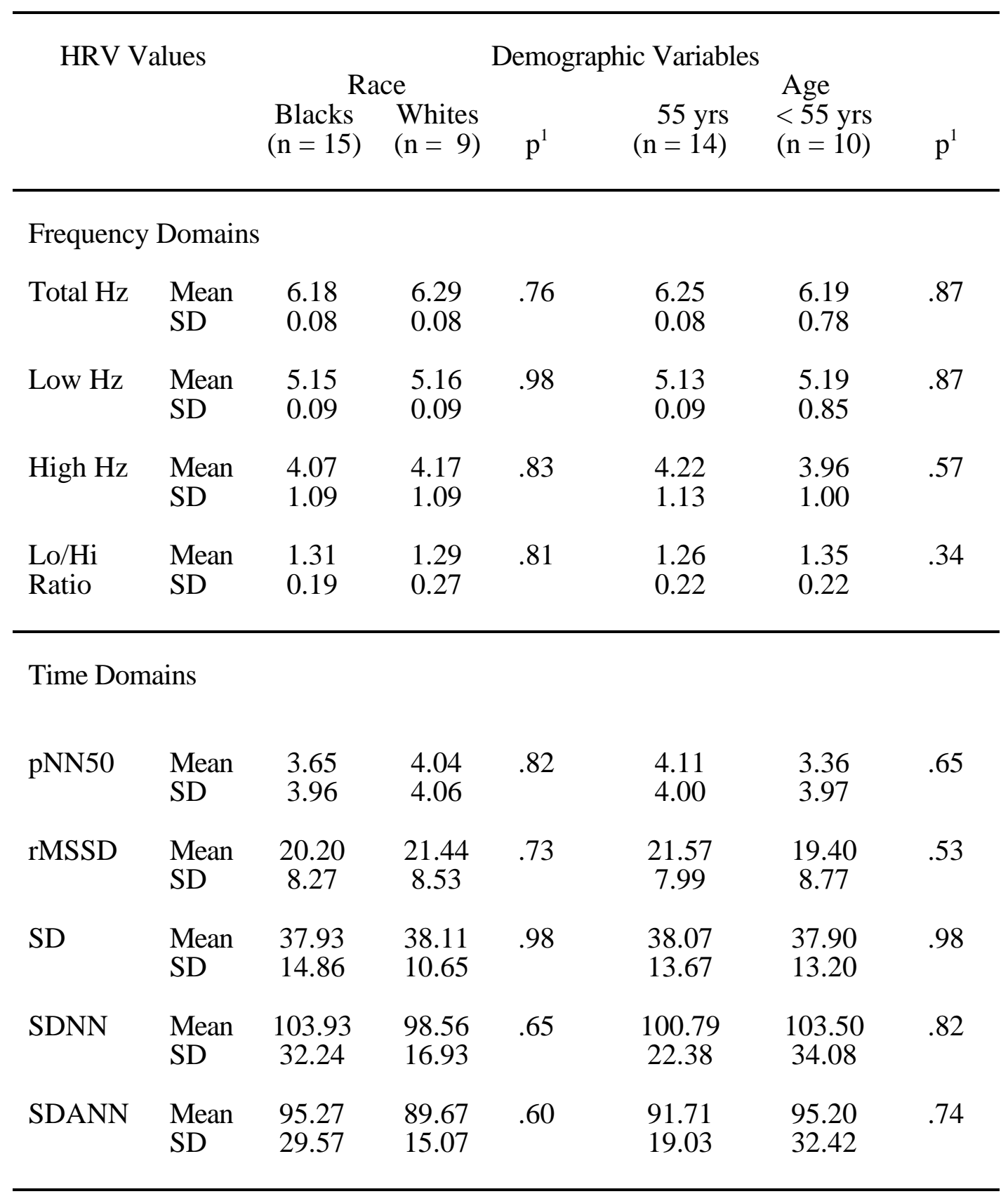

${ }^{1} \mathrm{t}$-Test: $\quad$ Significant at $\mathrm{p}=\leq .05 \quad$ Trend at $\mathrm{p}=\leq .10>.05$ 
Table 17

Comparisons of Pre-Test Mean Values for Heart Rate Variability for

Postmenopausal Women Differing Across Medical Variables of Diabetes Mellitus and Hypertension

\begin{tabular}{|c|c|c|c|c|c|c|c|}
\hline \multirow{2}{*}{\multicolumn{2}{|c|}{ HRV Variables }} & \multicolumn{6}{|c|}{ Medical Variables } \\
\hline & & \multicolumn{2}{|c|}{ Diabetes Mellitus } & & \multicolumn{3}{|c|}{ Hypertension } \\
\hline & & $\begin{array}{c}\text { Absent } \\
(\mathrm{n}=16)\end{array}$ & $\begin{array}{l}\text { Present } \\
(\mathrm{n}=8)\end{array}$ & $\mathrm{p}^{1}$ & $\begin{array}{l}\text { Absent } \\
(\mathrm{n}=13)\end{array}$ & $\begin{array}{l}\text { Present } \\
(\mathrm{n}=11)\end{array}$ & $\mathrm{p}^{1}$ \\
\hline \multicolumn{8}{|c|}{ Frequency Domains } \\
\hline \multirow[t]{2}{*}{ Total Hz } & Mean & 6.16 & 6.34 & \multirow[t]{2}{*}{.62} & 6.48 & 5.92 & \multirow[t]{2}{*}{.09} \\
\hline & $\mathrm{SD}$ & 0.81 & 6.57 & & 0.53 & 0.96 & \\
\hline \multirow[t]{2}{*}{ Low $\mathrm{Hz}$} & Mean & 5.07 & 5.33 & \multirow[t]{2}{*}{.51} & 5.52 & 4.72 & \multirow[t]{2}{*}{.02} \\
\hline & $\mathrm{SD}$ & 0.91 & 0.87 & & 0.62 & 0.98 & \\
\hline \multirow[t]{2}{*}{ High Hz } & Mean & 4.10 & 4.12 & \multirow[t]{2}{*}{.97} & 4.28 & 3.91 & \multirow[t]{2}{*}{.41} \\
\hline & $\mathrm{SD}$ & 1.05 & 1.17 & & 0.82 & 1.31 & \\
\hline $\mathrm{Lo} / \mathrm{Hi}$ & Mean & 1.27 & 1.36 & \multirow[t]{2}{*}{.38} & 1.32 & 1.28 & \multirow[t]{2}{*}{.68} \\
\hline Ratio & $\mathrm{SD}$ & 0.19 & 0.28 & & 0.19 & 0.26 & \\
\hline \multicolumn{8}{|c|}{ Time Domains } \\
\hline \multirow[t]{2}{*}{ pNN50 } & Mean & 3.36 & 4.69 & \multirow[t]{2}{*}{.44} & 4.40 & 3.09 & \multirow[t]{2}{*}{.43} \\
\hline & SD & 3.26 & 5.12 & & 4.27 & 3.50 & \\
\hline \multirow[t]{2}{*}{ rMSSD } & Mean & 20.13 & 21.75 & \multirow[t]{2}{*}{.66} & 22.69 & 18.27 & \multirow[t]{2}{*}{.20} \\
\hline & SD & 7.40 & 10.10 & & 8.35 & 7.72 & \\
\hline \multirow[t]{2}{*}{ SD } & Mean & 37.25 & 39.50 & \multirow[t]{2}{*}{.70} & 40.85 & 34.64 & \multirow[t]{2}{*}{.26} \\
\hline & SD & 12.40 & 15.43 & & 9.79 & 16.18 & \\
\hline \multirow[t]{2}{*}{ SDNN } & Mean & 101.88 & 102.00 & \multirow[t]{2}{*}{.61} & 104.62 & 98.73 & \multirow[t]{2}{*}{.61} \\
\hline & SD & 28.45 & 26.37 & & 24.80 & 30.70 & \\
\hline \multirow[t]{2}{*}{ SDANN } & Mean & 93.63 & 92.25 & \multirow[t]{2}{*}{.90} & 94.23 & 91.91 & \multirow[t]{2}{*}{.83} \\
\hline & $\mathrm{SD}$ & 26.24 & 23.61 & & 24.01 & 26.00 & \\
\hline
\end{tabular}


Table 18

Comparisons of Pre-Test Mean Values for Heart Rate Variability for

Postmenopausal Women Differing Across Social Variables of Smoking Status and

Planned Exercise

\begin{tabular}{|c|c|c|c|c|c|c|c|}
\hline \multirow{2}{*}{\multicolumn{2}{|c|}{ HRV Variables }} & \multicolumn{6}{|c|}{ Social Variables } \\
\hline & & \multicolumn{2}{|c|}{ Smoking Status } & & \multicolumn{2}{|c|}{ Planned Exercise } & \multirow[b]{2}{*}{$\mathrm{p}^{1}$} \\
\hline & & $\begin{array}{c}\text { Absent } \\
(n=15)\end{array}$ & $\begin{array}{l}\text { Present } \\
(n=9)\end{array}$ & $\mathrm{p}^{1}$ & $\begin{array}{c}\text { Absent } \\
(\mathrm{n}=11)\end{array}$ & $\begin{array}{c}\text { Present } \\
(\mathrm{n}=13)\end{array}$ & \\
\hline \multicolumn{8}{|c|}{ Frequency Domains } \\
\hline \multirow{2}{*}{ Total Hz } & Mean & 6.29 & 6.10 & \multirow{2}{*}{.58} & 6.28 & 6.17 & \multirow[t]{2}{*}{.75} \\
\hline & $\mathrm{SD}$ & 0.83 & 0.77 & & 0.75 & 0.86 & \\
\hline \multirow{2}{*}{ Low $\mathrm{Hz}$} & Mean & 5.18 & 5.10 & \multirow{2}{*}{.53} & 5.28 & 5.05 & \multirow[t]{2}{*}{.53} \\
\hline & $\mathrm{SD}$ & 0.92 & 0.87 & & 0.83 & 0.95 & \\
\hline \multirow{2}{*}{ High Hz } & Mean & 4.29 & 3.81 & \multirow{2}{*}{.30} & 4.19 & 4.04 & \multirow{2}{*}{.73} \\
\hline & $\mathrm{SD}$ & 1.03 & 1.12 & & 1.04 & 1.12 & \\
\hline $\mathrm{Lo} / \mathrm{Hi}$ & Mean & 1.24 & 1.40 & \multirow{2}{*}{.09} & 1.30 & 1.30 & \multirow[t]{2}{*}{.99} \\
\hline Ratio & SD & 0.18 & 0.25 & & 0.20 & 0.25 & \\
\hline \multicolumn{8}{|c|}{ Time Domains } \\
\hline \multirow[t]{2}{*}{ pNN50 } & Mean & 4.33 & 2.92 & \multirow[t]{2}{*}{.41} & 3.51 & 4.05 & \multirow[t]{2}{*}{.75} \\
\hline & $\mathrm{SD}$ & 3.82 & 4.14 & & 3.66 & 4.25 & \\
\hline \multirow[t]{2}{*}{ rMSSD } & Mean & 21.87 & 18.67 & \multirow{2}{*}{.37} & 20.45 & 20.85 & \multirow[t]{2}{*}{.91} \\
\hline & SD & 7.76 & 9.00 & & 7.81 & 8.84 & \\
\hline \multirow[t]{2}{*}{ SD } & Mean & 39.60 & 35.33 & \multirow{2}{*}{.46} & 37.45 & 38.46 & \multirow[t]{2}{*}{.86} \\
\hline & $\mathrm{SD}$ & 13.68 & 12.63 & & 11.82 & 14.71 & \\
\hline \multirow[t]{2}{*}{ SDNN } & Mean & 102.00 & 101.78 & \multirow{2}{*}{.99} & 104.18 & 100.00 & \multirow[t]{2}{*}{.72} \\
\hline & $\mathrm{SD}$ & 26.35 & 30.19 & & 29.02 & 26.59 & \\
\hline \multirow[t]{2}{*}{ SDANN } & Mean & 92.80 & 93.78 & \multirow[t]{2}{*}{.93} & 96.27 & 90.54 & \multirow[t]{2}{*}{.59} \\
\hline & SD & 24.02 & 27.74 & & 27.62 & 23.12 & \\
\hline
\end{tabular}


Table 19

Comparisons of Pre-Test Mean Values for Heart Rate Variability for

Postmenopausal Women Differing in Level of Health Risk Across Medical

Variable of Body Mass Index

\begin{tabular}{|c|c|c|c|c|}
\hline \multicolumn{2}{|c|}{ HRV Values } & $\begin{array}{c}\text { BMI }<27 \\
\text { Low/Moderate } \\
\text { Health Risk } \\
(\mathrm{n}=12)\end{array}$ & $\begin{array}{c}\text { BMI } \geq 27 \\
\text { High/Very High } \\
\text { Health Risk } \\
(\mathrm{n}=12)\end{array}$ & $\mathrm{p}^{1}$ \\
\hline \multicolumn{5}{|c|}{ Frequency Domains } \\
\hline Total Hz & $\begin{array}{l}\text { Mean } \\
\text { SD }\end{array}$ & $\begin{array}{l}6.05 \\
0.92\end{array}$ & $\begin{array}{l}6.39 \\
0.65\end{array}$ & .31 \\
\hline Low $\mathrm{Hz}$ & $\begin{array}{l}\text { Mean } \\
\text { SD }\end{array}$ & $\begin{array}{l}4.93 \\
1.01\end{array}$ & $\begin{array}{l}5.38 \\
0.71\end{array}$ & .22 \\
\hline High Hz & $\begin{array}{l}\text { Mean } \\
\text { SD }\end{array}$ & $\begin{array}{l}3.98 \\
1.19\end{array}$ & $\begin{array}{l}4.23 \\
0.96\end{array}$ & .73 \\
\hline Lo/Hi Ratio & $\begin{array}{l}\text { Mean } \\
\text { SD }\end{array}$ & $\begin{array}{l}1.28 \\
0.21\end{array}$ & $\begin{array}{l}1.32 \\
0.23\end{array}$ & \\
\hline \multicolumn{5}{|c|}{ Time Domains } \\
\hline pNN50 & $\begin{array}{l}\text { Mean } \\
\text { SD }\end{array}$ & $\begin{array}{l}3.25 \\
3.72\end{array}$ & $\begin{array}{l}4.35 \\
4.19\end{array}$ & .50 \\
\hline rMSSD & $\begin{array}{l}\text { Mean } \\
\text { SD }\end{array}$ & $\begin{array}{c}19.50 \\
8.40\end{array}$ & $\begin{array}{c}21.83 \\
8.20\end{array}$ & .50 \\
\hline SD & $\begin{array}{l}\text { Mean } \\
\text { SD }\end{array}$ & $\begin{array}{l}36.17 \\
14.21\end{array}$ & $\begin{array}{l}39.83 \\
12.42\end{array}$ & .51 \\
\hline SDNN & $\begin{array}{l}\text { Mean } \\
\text { SD }\end{array}$ & $\begin{array}{l}97.17 \\
30.36\end{array}$ & $\begin{array}{c}106.67 \\
23.99\end{array}$ & .40 \\
\hline SDANN & $\begin{array}{l}\text { Mean } \\
\text { SD }\end{array}$ & $\begin{array}{l}89.17 \\
27.21\end{array}$ & $\begin{array}{l}97.19 \\
22.78\end{array}$ & .44 \\
\hline
\end{tabular}

${ }^{1} \mathrm{t}$-Test: $\quad$ Significant at $\mathrm{p}=\leq .05 \quad$ Trend at $\mathrm{p}=\leq .10>.05$ 
Table 20

Comparison of Pre-Test Mean Values for Heart Rate Variability for

Postmenopausal Women Differing Across Reproductive Variables of Age at

Menopause and Years of Menopause

\begin{tabular}{|c|c|c|c|c|c|c|c|}
\hline \multirow{2}{*}{\multicolumn{2}{|c|}{ HRV Variables }} & \multicolumn{6}{|c|}{$\begin{array}{l}\text { Reproductive Variables } \\
\text { Number of Years o } \\
\text { enopause }\end{array}$} \\
\hline & & $\begin{array}{c}\geq 51 \text { yrs } \\
(\mathrm{n}=7)\end{array}$ & $\begin{array}{l}<51 \text { yrs } \\
(\mathrm{n}=17)\end{array}$ & $\mathrm{p}^{1}$ & $\begin{array}{l}\geq 9 \text { yrs } \\
(\mathrm{n}=11)\end{array}$ & $\begin{array}{l}<9 \text { yrs } \\
(n=13)\end{array}$ & $\mathrm{p}^{1}$ \\
\hline \multicolumn{8}{|c|}{ Frequency Domains } \\
\hline \multirow[t]{2}{*}{ Total Hz } & Mean & 6.31 & 6.19 & \multirow[t]{2}{*}{.74} & 5.85 & 6.54 & \multirow[t]{2}{*}{.03} \\
\hline & SD & 0.84 & 0.80 & & 0.76 & 0.70 & \\
\hline \multirow[t]{2}{*}{ Low $\mathrm{Hz}$} & Mean & 5.18 & 5.14 & \multirow[t]{2}{*}{.93} & 4.68 & 5.56 & \multirow[t]{2}{*}{.01} \\
\hline & $\mathrm{SD}$ & 0.97 & 0.88 & & 0.80 & 0.76 & \\
\hline \multirow[t]{2}{*}{ High $\mathrm{Hz}$} & Mean & 4.29 & 4.03 & \multirow[t]{2}{*}{.61} & 3.69 & 4.46 & \multirow[t]{2}{*}{.06} \\
\hline & SD & 1.26 & 1.01 & & 1.11 & 0.92 & \\
\hline $\mathrm{Lo} / \mathrm{Hi}$ & Mean & 1.28 & 1.31 & \multirow[t]{2}{*}{.74} & 1.33 & 1.27 & \multirow[t]{2}{*}{.54} \\
\hline Ratio & $\mathrm{SD}$ & 0.30 & 0.19 & & 0.27 & 0.18 & \\
\hline \multicolumn{8}{|c|}{ Time Domains } \\
\hline \multirow[t]{2}{*}{ pNN50 } & Mean & 3.33 & 3.99 & \multirow[t]{2}{*}{.71} & 2.52 & 4.88 & \multirow[t]{2}{*}{.14} \\
\hline & SD & 2.16 & 4.49 & & 3.86 & 3.77 & \\
\hline \multirow[t]{2}{*}{ rMSSD } & Mean & 20.14 & 20.88 & \multirow[t]{2}{*}{.85} & 17.64 & 23.23 & \multirow[t]{2}{*}{.09} \\
\hline & SD & 6.15 & 9.08 & & 8.46 & 7.34 & \\
\hline \multirow[t]{2}{*}{ SD } & Mean & 37.71 & 38.12 & \multirow[t]{2}{*}{.95} & 31.64 & 43.38 & \multirow[t]{2}{*}{.03} \\
\hline & SD & 11.84 & 14.04 & & 9.67 & 13.67 & \\
\hline \multirow[t]{2}{*}{ SDNN } & Mean & 93.00 & 105.59 & \multirow[t]{2}{*}{.17} & 93.55 & 109.00 & \multirow[t]{2}{*}{.17} \\
\hline & SD & 16.16 & 31.84 & & 18.50 & 31.84 & \\
\hline \multirow[t]{2}{*}{ SDANN } & Mean & 83.71 & 97.06 & \multirow[t]{2}{*}{.25} & 87.27 & 98.15 & \multirow[t]{2}{*}{.30} \\
\hline & SD & 14.94 & 27.40 & & 16.69 & 29.93 & \\
\hline
\end{tabular}

${ }^{1} \mathrm{t}$-Test: $\quad$ Significant at $\mathrm{p}=\leq .05 \quad$ Trend at $\mathrm{p}=\leq .10>.05$ 
Appendix I

Data Tables: Post-Test Heart Rate Variability for

Postmenopausal Women Across Selected Cardiovascular Risk Factors 
Table 21

Comparisons of Post-Test Mean Values for Heart Rate Variability for

Postmenopausal Women Differing Across Demographic Variables of Race and Age

\begin{tabular}{|c|c|c|c|c|c|c|c|}
\hline \multirow{2}{*}{\multicolumn{2}{|c|}{ HRV Variables }} & \multicolumn{6}{|c|}{ Demographic Variables } \\
\hline & & \multicolumn{2}{|c|}{ Race } & & \multicolumn{2}{|c|}{ Age $($ Mean=54.9) } & \multirow[b]{2}{*}{$\mathrm{p}^{1}$} \\
\hline & & $\begin{array}{l}\text { Blacks } \\
(\mathrm{n}=15)\end{array}$ & $\begin{array}{l}\text { Whites } \\
(\mathrm{n}=9)\end{array}$ & $\mathrm{p}^{1}$ & $\begin{array}{l}\geq 55 \text { yrs } \\
(\mathrm{n}=14)\end{array}$ & $\begin{array}{l}<55 \text { yrs } \\
(\mathrm{n}=10)\end{array}$ & \\
\hline \multicolumn{8}{|c|}{ Frequency Domains } \\
\hline \multirow[t]{2}{*}{ Total Hz } & Mean & 6.49 & 6.19 & \multirow[t]{2}{*}{.37} & 6.31 & 6.49 & \multirow[t]{2}{*}{.58} \\
\hline & SD & 0.64 & 0.98 & & 0.89 & 0.63 & \\
\hline \multirow[t]{2}{*}{ Low $\mathrm{Hz}$} & Mean & 5.47 & 5.26 & \multirow[t]{2}{*}{.54} & 5.28 & 5.54 & \multirow[t]{2}{*}{.46} \\
\hline & SD & 0.69 & 1.04 & & 0.93 & 0.66 & \\
\hline \multirow[t]{2}{*}{ High Hz } & Mean & 4.40 & 3.90 & \multirow[t]{2}{*}{.27} & 4.15 & 4.30 & \multirow[t]{2}{*}{.74} \\
\hline & SD & 0.91 & 1.28 & & 1.20 & 0.90 & \\
\hline $\mathrm{Lo} / \mathrm{Hi}$ & Mean & 1.27 & 1.42 & \multirow[t]{2}{*}{.13} & 1.33 & 1.32 & \multirow[t]{2}{*}{.90} \\
\hline Ratio & SD & 0.19 & 0.28 & & 0.24 & 0.24 & \\
\hline \multicolumn{8}{|c|}{ Time Domains } \\
\hline \multirow[t]{2}{*}{ pNN50 } & Mean & 4.47 & 4.69 & \multirow[t]{2}{*}{.92} & 5.29 & 3.53 & \multirow[t]{2}{*}{.42} \\
\hline & SD & 4.72 & 6.10 & & 5.90 & 3.94 & \\
\hline \multirow[t]{2}{*}{ rMSSD } & Mean & 22.13 & 21.33 & \multirow[t]{2}{*}{.86} & 22.79 & 20.50 & \multirow[t]{2}{*}{.60} \\
\hline & SD & 8.99 & 12.45 & & 11.16 & 8.98 & \\
\hline \multirow[t]{2}{*}{ SD } & Mean & 40.27 & 38.11 & \multirow[t]{2}{*}{.70} & 39.29 & 39.70 & \multirow[t]{2}{*}{.94} \\
\hline & SD & 12.03 & 14.47 & & 13.30 & 12.59 & \\
\hline \multirow[t]{2}{*}{ SDNN } & Mean & 100.40 & 95.00 & \multirow[t]{2}{*}{.65} & 93.14 & 105.70 & \multirow[t]{2}{*}{.28} \\
\hline & SD & 30.99 & 20.92 & & 21.70 & 33.44 & \\
\hline \multirow[t]{2}{*}{ SDANN } & Mean & 91.00 & 84.56 & \multirow[t]{2}{*}{.58} & 82.86 & 96.60 & \multirow[t]{2}{*}{.22} \\
\hline & $\mathrm{SD}$ & 30.79 & 18.64 & & 20.13 & 33.20 & \\
\hline
\end{tabular}

${ }^{1} \mathrm{t}$-Test: $\quad$ Significant at $\mathrm{p}=\leq .05 \quad$ Trend at $\mathrm{p}=\leq .10>.05$ 
Table 22

Comparisons of Post-Test Mean Values for Heart Rate Variability for

Postmenopausal Women Differing Across Medical Variables of Diabetes Mellitus and Hypertension

\begin{tabular}{|c|c|c|c|c|c|c|c|}
\hline \multirow{3}{*}{\multicolumn{2}{|c|}{ HRV Variables }} & \multicolumn{6}{|c|}{ Medical Variables } \\
\hline & & \multicolumn{2}{|c|}{ Diabetes Mellitus } & & \multicolumn{2}{|c|}{ Hypertension } & \multirow[b]{2}{*}{$\mathrm{p}^{1}$} \\
\hline & & $\begin{array}{c}\text { Absent } \\
(\mathrm{n}= \\
16)\end{array}$ & $\begin{array}{l}\text { Present } \\
(\mathrm{n}=8)\end{array}$ & $\mathrm{p}^{1}$ & $\begin{array}{l}\text { Absent } \\
(n=13)\end{array}$ & $\begin{array}{l}\text { Present } \\
(\mathrm{n}=11)\end{array}$ & \\
\hline \multicolumn{8}{|c|}{ Frequency Domains } \\
\hline \multirow[t]{2}{*}{ Total Hz } & Mean & 6.42 & 6.31 & \multirow[t]{2}{*}{.76} & 6.56 & 6.17 & \multirow[t]{2}{*}{.22} \\
\hline & $\mathrm{SD}$ & 0.81 & 0.77 & & 0.69 & 0.86 & \\
\hline \multirow[t]{2}{*}{ Low $\mathrm{Hz}$} & Mean & 5.43 & 5.32 & \multirow[t]{2}{*}{.78} & 5.66 & 5.07 & \multirow[t]{2}{*}{0.08} \\
\hline & $\mathrm{SD}$ & 0.88 & 0.75 & & 0.66 & 0.91 & \\
\hline \multirow[t]{2}{*}{ High Hz } & Mean & 4.27 & 4.12 & \multirow[t]{2}{*}{.76} & 4.32 & 4.09 & \multirow[t]{2}{*}{.61} \\
\hline & $\mathrm{SD}$ & 0.99 & 1.27 & & 1.01 & 1.17 & \\
\hline $\mathrm{Lo} / \mathrm{Hi}$ & Mean & 1.30 & 1.38 & \multirow[t]{2}{*}{.47} & 1.35 & 1.30 & \multirow[t]{2}{*}{.56} \\
\hline Ratio & SD & 0.16 & 0.34 & & 0.23 & 0.25 & \\
\hline \multicolumn{8}{|c|}{ Time Domains } \\
\hline \multirow[t]{2}{*}{ pNN50 } & Mean & 3.66 & 6.35 & \multirow[t]{2}{*}{.24} & 4.53 & 4.58 & \multirow[t]{2}{*}{.98} \\
\hline & SD & 3.42 & 7.52 & & 5.70 & 4.70 & \\
\hline \multirow[t]{2}{*}{ rMSSD } & Mean & 21.06 & 23.38 & \multirow[t]{2}{*}{.61} & 22.62 & 20.91 & \multirow[t]{2}{*}{.69} \\
\hline & SD & 8.28 & 13.72 & & 11.23 & 9.19 & \\
\hline \multirow[t]{2}{*}{ SD } & Mean & 40.25 & 37.88 & \multirow[t]{2}{*}{.68} & 41.46 & 37.09 & \multirow[t]{2}{*}{.41} \\
\hline & SD & 12.26 & 14.35 & & 12.00 & 13.72 & \\
\hline \multirow[t]{2}{*}{ SDNN } & Mean & 100.19 & 94.75 & \multirow[t]{2}{*}{.66} & 103.08 & 92.82 & \multirow[t]{2}{*}{.37} \\
\hline & SD & 28.30 & 26.56 & & 24.65 & 30.31 & \\
\hline \multirow[t]{2}{*}{ SDANN } & Mean & 90.38 & 85.00 & \multirow[t]{2}{*}{.65} & 93.69 & 82.55 & \multirow[t]{2}{*}{.32} \\
\hline & SD & 28.31 & 24.25 & & 24.05 & 29.34 & \\
\hline
\end{tabular}

${ }^{1} \mathrm{t}$-Test: $\quad$ Significant at $\mathrm{p}=\leq .05 \quad$ Trend at $\mathrm{p}=\leq .10>.05$ 
Table 23

Comparisons of Post-Test Mean Values for Heart Rate Variability for

Postmenopausal Women Differing Across Social Variables of Smoking Status and Planned Exercise

\begin{tabular}{|c|c|c|c|c|c|c|c|}
\hline \multirow{3}{*}{\multicolumn{2}{|c|}{ HRV Variables }} & \multicolumn{6}{|c|}{ Variables } \\
\hline & & \multicolumn{2}{|c|}{ Smoking Status } & & \multicolumn{2}{|c|}{ Planned Exercise } & \multirow[b]{2}{*}{$\mathrm{p}^{1}$} \\
\hline & & $\begin{array}{c}\text { Absent } \\
(\mathrm{n}= \\
15)\end{array}$ & $\begin{array}{l}\text { Present } \\
(\mathrm{n}=9)\end{array}$ & $\mathrm{p}^{1}$ & $\begin{array}{c}\text { Absent } \\
(\mathrm{n}= \\
11)\end{array}$ & $\begin{array}{l}\text { Present } \\
(\mathrm{n}=13)\end{array}$ & \\
\hline \multicolumn{8}{|c|}{ Frequency Domains } \\
\hline \multirow[t]{2}{*}{ Total Hz } & Mean & 6.52 & 6.15 & \multirow[t]{2}{*}{.26} & 6.35 & 6.41 & \multirow[t]{2}{*}{.85} \\
\hline & $\mathrm{SD}$ & 0.78 & 0.77 & & 0.74 & 0.84 & \\
\hline \multirow[t]{2}{*}{ Low $\mathrm{Hz}$} & Mean & 5.50 & 5.21 & \multirow[t]{2}{*}{.41} & 5.35 & 5.43 & \multirow[t]{2}{*}{.81} \\
\hline & $\mathrm{SD}$ & 0.85 & 0.79 & & 0.75 & 0.91 & \\
\hline \multirow[t]{2}{*}{ High $\mathrm{Hz}$} & Mean & 4.49 & 3.76 & \multirow[t]{2}{*}{.11} & 4.19 & 4.23 & \multirow[t]{2}{*}{.93} \\
\hline & $\mathrm{SD}$ & 0.93 & 1.18 & & 1.14 & 1.05 & \\
\hline $\mathrm{Lo} / \mathrm{Hi}$ & Mean & 1.25 & 1.45 & \multirow[t]{2}{*}{.05} & 1.32 & 1.33 & \multirow[t]{2}{*}{.96} \\
\hline Ratio & $\mathrm{SD}$ & 0.16 & 0.29 & & 0.24 & 0.24 & \\
\hline \multicolumn{8}{|c|}{ Time Domains } \\
\hline \multirow[t]{2}{*}{ pNN50 } & Mean & 5.50 & 2.98 & \multirow[t]{2}{*}{.25} & 4.24 & 4.82 & \multirow[t]{2}{*}{.79} \\
\hline & $\mathrm{SD}$ & 5.49 & 4.36 & & 4.53 & 5.79 & \\
\hline \multirow[t]{2}{*}{ rMSSD } & Mean & 23.67 & 18.78 & \multirow[t]{2}{*}{.26} & 21.73 & 21.93 & \multirow[t]{2}{*}{.96} \\
\hline & $\mathrm{SD}$ & 9.85 & 10.51 & & 10.16 & 10.58 & \\
\hline \multirow[t]{2}{*}{$\mathrm{SD}$} & Mean & 42.00 & 35.22 & \multirow[t]{2}{*}{.21} & 39.09 & 39.77 & \multirow[t]{2}{*}{.90} \\
\hline & $\mathrm{SD}$ & 12.48 & 12.69 & & 12.54 & 13.38 & \\
\hline \multirow[t]{2}{*}{ SDNN } & Mean & 101.93 & 92.44 & \multirow[t]{2}{*}{.42} & 101.91 & 95.38 & \multirow[t]{2}{*}{.57} \\
\hline & $\mathrm{SD}$ & 27.68 & 27.09 & & 32.67 & 22.69 & \\
\hline \multirow[t]{2}{*}{ SDANN } & Mean & 91.33 & 84.00 & \multirow[t]{2}{*}{.53} & 92.36 & 85.38 & \multirow[t]{2}{*}{.53} \\
\hline & $\mathrm{SD}$ & 27.63 & 25.73 & & 31.71 & 22.25 & \\
\hline
\end{tabular}

${ }^{1} \mathrm{t}$-Test: $\quad$ Significant at $\mathrm{p}=\leq .05 \quad$ Trend at $\mathrm{p}=\leq .10>.05$ 
Table 24

Comparisons of Post-Test Mean Values for Heart Rate Variability for

Postmenopausal Women Differing in Level of Health Risk Across the Medical

Variable of Body Mass Index

\begin{tabular}{|c|c|c|c|c|}
\hline \multicolumn{2}{|c|}{ HRV Values } & $\begin{array}{c}\text { BMI < } 27 \\
\text { Low/Moderate } \\
\text { Health Risk } \\
(\mathrm{n}=12)\end{array}$ & $\begin{array}{c}\text { BMI } \geq 27 \\
\text { High/Very High } \\
\text { Health Risk } \\
(n=12)\end{array}$ & $\mathrm{p}^{1}$ \\
\hline \multicolumn{5}{|c|}{ Frequency Domains } \\
\hline Total Hz & $\begin{array}{l}\text { Mean } \\
\text { SD }\end{array}$ & $\begin{array}{l}6.32 \\
0.91\end{array}$ & $\begin{array}{l}6.44 \\
0.66\end{array}$ & .71 \\
\hline Low $\mathrm{Hz}$ & $\begin{array}{l}\text { Mean } \\
\text { SD }\end{array}$ & $\begin{array}{l}5.36 \\
1.00\end{array}$ & $\begin{array}{l}5.42 \\
0.64\end{array}$ & .85 \\
\hline High Hz & $\begin{array}{l}\text { Mean } \\
\text { SD }\end{array}$ & $\begin{array}{l}4.14 \\
1.12\end{array}$ & $\begin{array}{l}4.29 \\
1.06\end{array}$ & .75 \\
\hline Lo/Hi Ratio & $\begin{array}{l}\text { Mean } \\
\text { SD }\end{array}$ & $\begin{array}{l}1.33 \\
0.17\end{array}$ & $\begin{array}{l}1.33 \\
0.29\end{array}$ & .97 \\
\hline \multicolumn{5}{|c|}{ Time Domains } \\
\hline pNN50 & $\begin{array}{l}\text { Mean } \\
\text { SD }\end{array}$ & $\begin{array}{l}3.39 \\
3.77\end{array}$ & $\begin{array}{l}5.72 \\
6.19\end{array}$ & .28 \\
\hline rMSSD & $\begin{array}{l}\text { Mean } \\
\text { SD }\end{array}$ & $\begin{array}{c}20.17 \\
9.21\end{array}$ & $\begin{array}{l}23.50 \\
11.18\end{array}$ & .43 \\
\hline $\mathrm{SD}$ & $\begin{array}{l}\text { Mean } \\
\text { SD }\end{array}$ & $\begin{array}{l}39.08 \\
13.91\end{array}$ & $\begin{array}{l}39.83 \\
12.04\end{array}$ & .89 \\
\hline SDNN & $\begin{array}{l}\text { Mean } \\
\text { SD }\end{array}$ & $\begin{array}{l}93.08 \\
24.72\end{array}$ & $\begin{array}{c}103.67 \\
29.72\end{array}$ & .35 \\
\hline SDANN & $\begin{array}{l}\text { Mean } \\
\text { SD }\end{array}$ & $\begin{array}{l}82.17 \\
22.34\end{array}$ & $\begin{array}{l}95.00 \\
29.87\end{array}$ & .25 \\
\hline
\end{tabular}

${ }^{1} \mathrm{t}$-Test: $\quad$ Significant at $\mathrm{p}=\leq .05 \quad$ Trend at $\mathrm{p}=\leq .10>.05$ 
Table 25

Comparisons of Post-Test Mean Values for Heart Rate Variability for

Postmenopausal Women Differing Across Reproductive Variables of Age at

Menopause and Years of Menopause

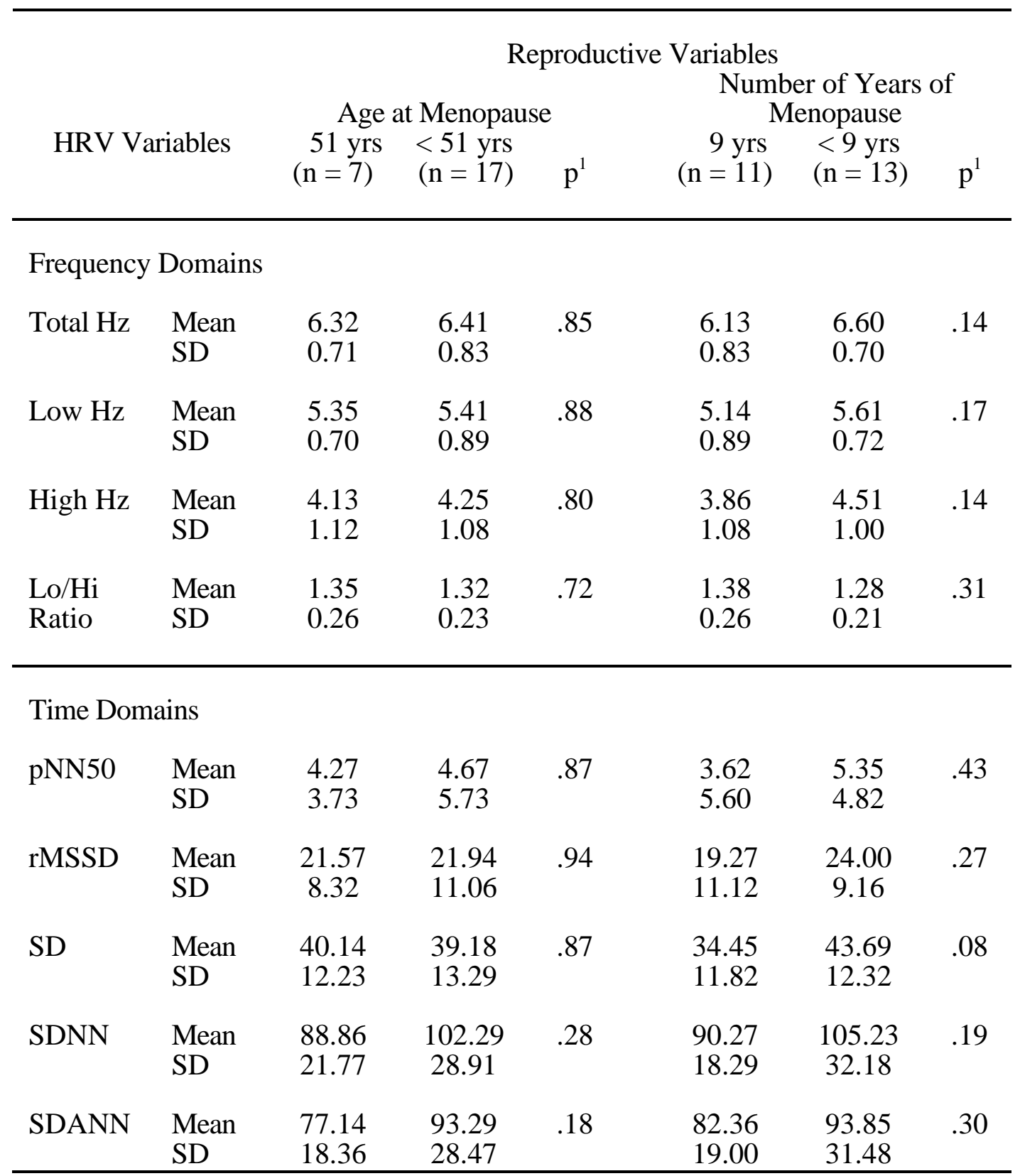

${ }^{1} \mathrm{t}$-Test: $\quad$ Significant at $\mathrm{p}=\leq .05 \quad$ Trend at $\mathrm{p}=\leq .10>.05$ 
Appendix J

Data Tables: Differences Between Pre- and Post-Test Heart Rate Variability for Postmenopausal Women Across Selected Cardiovascular Risk Factors 
Table 26

Differences Between Pre- and Post-Test Mean Values for Heart Rate Variability in Postmenopausal Women Across and Within Demographic Variable of Age

HRV Variables

Demographic Variables

Age $\geq 55$ years $(n=14)$ Age $<55$ years $(n=10)$

Pre-Test Post-Test $\mathrm{p}^{1} \quad$ Pre-Test Post-Test $\mathrm{p}^{1}$

Frequency Domains

$\begin{array}{llllllll}\text { Total Hz } & \text { Mean } & 6.25 & 6.31 & .68 & 6.19 & 6.49 & .06 \\ & \text { SD } & 0.08 & 0.89 & & 0.78 & 0.63 & \\ \text { Low Hz } & \text { Mean } & 5.13 & 5.28 & .39 & 5.19 & 5.54 & .05 \\ & \text { SD } & 0.09 & 0.93 & & 0.85 & 0.66 & \\ \text { High Hz } & \text { Mean } & 4.22 & 4.15 & \downarrow .72 & 3.96 & 4.30 & .07 \\ & \text { SD } & 1.13 & 1.20 & & 1.00 & 0.90 & \\ \text { Lo/Hi } & \text { Mean } & 1.26 & 1.33 & \uparrow .14 & 1.35 & 1.32 & \downarrow .39 \\ \text { Ratio } & \text { SD } & 0.22 & 0.24 & & 0.22 & 0.24 & \end{array}$

Time Domains

$\begin{array}{llcccccc}\text { pNN50 } & \text { Mean } & 4.11 & 5.29 & .11 & 3.36 & 3.53 & .71 \\ & \text { SD } & 4.00 & 5.90 & & 3.97 & 3.94 & \\ \text { rMSSD } & \text { Mean } & 21.57 & 22.79 & .38 & 19.40 & 20.50 & .30 \\ & \text { SD } & 7.99 & 11.16 & & 8.77 & 8.98 & \\ \text { SD } & \text { Mean } & 38.07 & 39.29 & .53 & 37.90 & 39.70 & .28 \\ & \text { SD } & 13.67 & 13.30 & & 13.20 & 12.59 & \\ \text { SDNN } & \text { Mean } & 100.79 & 93.14 & \downarrow .06 & 103.50 & 105.70 & .62 \\ & \text { SD } & 22.38 & 21.70 & & 34.08 & 33.44 & \\ \text { SDANN } & \text { Mean } & 91.71 & 82.86 & \downarrow .05 & 95.20 & 96.60 & .79 \\ & \text { SD } & 19.03 & 20.13 & & 32.42 & 33.20 & \end{array}$

${ }^{1}$ Paired t-Test: $\quad$ Significant at $\mathrm{p}=\leq .05 \quad$ Trend at $\mathrm{p}=\leq .10>.05$ 
Table 27

Differences Between Pre- and Post-Test Mean Values for Heart Rate Variability in Postmenopausal Women By Absence/Presence of Diabetes Mellitus

\begin{tabular}{|c|c|c|c|c|c|c|c|}
\hline \multirow{3}{*}{\multicolumn{2}{|c|}{ HRV Variables }} & \multicolumn{6}{|c|}{ Medical Variables } \\
\hline & & \multicolumn{3}{|c|}{ Diabetes-Absent $(\mathrm{n}=16)$} & \multicolumn{3}{|c|}{ Diabetes-Present $(\mathrm{n}=8)$} \\
\hline & & Pre-Test & Post-Test & $\mathrm{p}^{1}$ & Pre-Test & Post-Test & $\mathrm{p}^{1}$ \\
\hline \multicolumn{8}{|c|}{ Frequency Domains } \\
\hline \multirow[t]{2}{*}{ Total Hz } & Mean & 6.16 & 6.42 & \multirow[t]{2}{*}{.03} & 6.34 & 6.31 & \multirow[t]{2}{*}{.89} \\
\hline & SD & 0.81 & 0.81 & & 6.57 & 0.77 & \\
\hline \multirow[t]{2}{*}{ Low $\mathrm{Hz}$} & Mean & 5.07 & 5.43 & \multirow[t]{2}{*}{.02} & 5.33 & 5.32 & \multirow[t]{2}{*}{.98} \\
\hline & SD & 0.91 & 0.88 & & 0.87 & 0.75 & \\
\hline \multirow[t]{2}{*}{ High Hz } & Mean & 4.10 & 4.27 & \multirow[t]{2}{*}{.32} & 4.12 & 4.12 & \multirow[t]{2}{*}{1.00} \\
\hline & $\mathrm{SD}$ & 1.05 & 0.99 & & 1.17 & 1.27 & \\
\hline $\mathrm{Lo} / \mathrm{Hi}$ & Mean & 1.27 & 1.30 & \multirow[t]{2}{*}{$\uparrow<.001$} & 1.36 & 1.38 & \multirow[t]{2}{*}{$\uparrow .66$} \\
\hline Ratio & $\mathrm{SD}$ & 0.19 & 0.16 & & 0.28 & 0.34 & \\
\hline \multicolumn{8}{|c|}{ Time Domains } \\
\hline \multirow[t]{2}{*}{ pNN50 } & Mean & 3.36 & 3.66 & \multirow[t]{2}{*}{.32} & 4.69 & 6.35 & \multirow[t]{2}{*}{.20} \\
\hline & SD & 3.26 & 3.42 & & 5.12 & 7.52 & \\
\hline \multirow[t]{2}{*}{ rMSSD } & Mean & 20.13 & 21.06 & \multirow[t]{2}{*}{.26} & 21.75 & 23.38 & \multirow[t]{2}{*}{.48} \\
\hline & SD & 7.40 & 8.28 & & 10.10 & 13.72 & \\
\hline \multirow[t]{2}{*}{ SD } & Mean & 37.25 & 40.25 & \multirow[t]{2}{*}{.02} & 39.50 & 37.88 & \multirow[t]{2}{*}{$\downarrow .57$} \\
\hline & SD & 12.40 & 12.26 & & 15.43 & 14.35 & \\
\hline \multirow[t]{2}{*}{ SDNN } & Mean & 101.88 & 100.19 & \multirow[t]{2}{*}{$\downarrow .60$} & 102.00 & 94.75 & \multirow[t]{2}{*}{$\downarrow .27$} \\
\hline & SD & 28.45 & 28.30 & & 26.37 & 26.56 & \\
\hline \multirow[t]{2}{*}{ SDANN } & Mean & 93.63 & 90.38 & \multirow[t]{2}{*}{$\downarrow .40$} & 92.25 & 85.00 & \multirow[t]{2}{*}{$\downarrow .30$} \\
\hline & SD & 26.24 & 28.31 & & 23.61 & 24.25 & \\
\hline
\end{tabular}

${ }^{1}$ Paired t-Test: $\quad$ Significant at $\mathrm{p}=\leq .05$

Trend at $\mathrm{p}=\leq .10>.05$ 
Table 28

Differences Between Pre- and Post-Test Mean Values for Heart Rate Variability for Postmenopausal Women By Smoking Status

\begin{tabular}{|c|c|c|c|c|c|c|c|}
\hline \multicolumn{2}{|c|}{ HRV Variables } & \multicolumn{6}{|c|}{ Social Variables } \\
\hline & & \multicolumn{3}{|c|}{ Non-Smoker $(\mathrm{n}=15)$} & \multicolumn{3}{|c|}{ Smoker $(\mathrm{n}=9)$} \\
\hline & & Pre-Test & Post-Test & $\mathrm{p}^{1}$ & Pre-Test & Post-Test & $\mathrm{p}^{1}$ \\
\hline \multicolumn{8}{|c|}{ Frequency Domains } \\
\hline \multirow[t]{2}{*}{ Total Hz } & Mean & 6.29 & 6.52 & .05 & 6.10 & 6.15 & .83 \\
\hline & SD & 0.83 & 0.78 & & 0.77 & 0.77 & \\
\hline \multirow[t]{2}{*}{ Low $\mathrm{Hz}$} & Mean & 5.18 & 5.50 & .03 & 5.10 & 5.21 & .66 \\
\hline & SD & 0.92 & 0.85 & & 0.87 & 0.79 & \\
\hline \multirow[t]{2}{*}{ High Hz } & Mean & 4.29 & 4.49 & .21 & 3.81 & 3.76 & $\downarrow .84$ \\
\hline & SD & 1.03 & 0.93 & & 1.12 & 1.18 & \\
\hline $\mathrm{Lo} / \mathrm{Hi}$ & Mean & 1.24 & 1.25 & $\uparrow .77$ & 1.40 & 1.45 & $\uparrow .22$ \\
\hline Ratio & SD & 0.18 & 0.16 & & 0.25 & 0.29 & \\
\hline \multicolumn{8}{|c|}{ Time Domains } \\
\hline \multirow[t]{2}{*}{ pNN50 } & Mean & 4.33 & 5.50 & .10 & 2.92 & 2.98 & .88 \\
\hline & SD & 3.82 & 5.49 & & 4.14 & 4.36 & \\
\hline \multirow[t]{2}{*}{ rMSSD } & Mean & 21.87 & 23.67 & .12 & 18.67 & 18.78 & .94 \\
\hline & SD & 7.76 & 9.85 & & 9.00 & 10.51 & \\
\hline \multirow[t]{2}{*}{ SD } & Mean & 39.60 & 42.00 & .08 & 35.33 & 35.22 & .97 \\
\hline & SD & 13.68 & 12.48 & & 12.63 & 12.69 & \\
\hline \multirow[t]{2}{*}{ SDNN } & Mean & 102.00 & 101.93 & $\downarrow .98$ & 101.78 & 92.44 & $\downarrow .09$ \\
\hline & SD & 26.35 & 27.68 & & 30.19 & 27.09 & \\
\hline \multirow[t]{2}{*}{ SDANN } & Mean & 92.80 & 91.33 & $\downarrow .70$ & 93.78 & 84.00 & $\downarrow .13$ \\
\hline & SD & 24.02 & 27.63 & & 27.74 & 25.73 & \\
\hline
\end{tabular}

${ }^{1}$ Paired t-Test: $\quad$ Significant at $\mathrm{p}=\leq .05$

Trend at $\mathrm{p}=\leq .10>.05$ 
Table 29

Differences Between Pre- and Post-Test Mean Values for Heart Rate Variability for Postmenopausal Women By Exercise Status

\begin{tabular}{|c|c|c|c|c|c|c|c|}
\hline \multirow{3}{*}{\multicolumn{2}{|c|}{ HRV Variables }} & \multicolumn{6}{|c|}{ Social Variables } \\
\hline & & \multicolumn{3}{|c|}{ Non-Exercisers $(\mathrm{n}=11)$} & \multicolumn{3}{|c|}{ Exercisers $(n=13)$} \\
\hline & & Pre-Test & Post-Test & $\mathrm{p}^{1}$ & Pre-Test & Post-Test & $\mathrm{p}^{1}$ \\
\hline \multicolumn{8}{|c|}{ Frequency } \\
\hline \multirow[t]{2}{*}{ Total Hz } & Mean & 6.28 & 6.35 & .70 & 6.17 & 6.41 & .07 \\
\hline & SD & 0.75 & 0.74 & & 0.86 & 0.84 & \\
\hline \multirow[t]{2}{*}{ Low $\mathrm{Hz}$} & Mean & 5.28 & 5.35 & .75 & 5.05 & 5.43 & .02 \\
\hline & SD & 0.83 & 0.75 & & 0.95 & 0.91 & \\
\hline \multirow[t]{2}{*}{ High Hz } & Mean & 4.19 & 4.19 & 1.00 & 4.04 & 4.23 & .28 \\
\hline & SD & 1.04 & 1.14 & & 1.12 & 1.05 & \\
\hline $\mathrm{Lo} / \mathrm{Hi}$ & Mean & 1.30 & 1.32 & $\uparrow .48$ & 1.30 & 1.33 & $\uparrow .57$ \\
\hline Ratio & SD & 0.20 & 0.24 & & 0.25 & 0.24 & \\
\hline \multicolumn{8}{|c|}{ Time Domains } \\
\hline \multirow[t]{2}{*}{ pNN50 } & Mean & 3.51 & 4.24 & .31 & 4.05 & 4.82 & .22 \\
\hline & SD & 3.66 & 4.53 & & 4.25 & 5.79 & \\
\hline \multirow[t]{2}{*}{ rMSSD } & Mean & 20.45 & 21.73 & .42 & 20.85 & 21.93 & .32 \\
\hline & SD & 7.81 & 10.16 & & 8.84 & 10.58 & \\
\hline \multirow[t]{2}{*}{ SD } & Mean & 37.45 & 39.09 & .45 & 38.46 & 39.77 & .42 \\
\hline & SD & 11.82 & 12.54 & & 14.71 & 13.38 & \\
\hline \multirow[t]{2}{*}{ SDNN } & Mean & 104.18 & 101.91 & $\downarrow .64$ & 100.00 & 95.38 & $\downarrow .24$ \\
\hline & SD & 29.02 & 32.67 & & 26.59 & 22.69 & \\
\hline \multirow[t]{2}{*}{ SDANN } & Mean & 96.27 & 92.36 & $\downarrow .42$ & 90.54 & 85.38 & $\downarrow .29$ \\
\hline & SD & 27.62 & 31.71 & & 23.12 & 22.25 & \\
\hline
\end{tabular}

${ }^{1}$ Paired t-Test: $\quad$ Significant at $\mathrm{p}=\leq .05 \quad$ Trend at $\mathrm{p}=\leq .10>.05$ 
Table 30

Differences Between Pre- and Post-Test Mean Values for Heart Rate Variability for Postmenopausal Women Across and Within the Medical Variable of Body Mass Index

\begin{tabular}{|c|c|c|c|c|c|c|c|}
\hline \multirow{2}{*}{\multicolumn{2}{|c|}{ HRV Variables }} & \multicolumn{6}{|c|}{ Body Mass Index } \\
\hline & & \multicolumn{3}{|c|}{$\mathrm{BMI}<27(\mathrm{n}=12)$} & \multicolumn{3}{|c|}{ BMI $\geq 27(n=12)$} \\
\hline \multicolumn{8}{|c|}{ Frequency Domains } \\
\hline \multirow[t]{2}{*}{ Total Hz } & Mean & 6.05 & 6.32 & \multirow[t]{2}{*}{.07} & 6.39 & 6.44 & \multirow[t]{2}{*}{.75} \\
\hline & $\mathrm{SD}$ & 0.92 & 0.91 & & 0.65 & 0.66 & \\
\hline \multirow[t]{2}{*}{ Low $\mathrm{Hz}$} & Mean & 4.93 & 5.36 & \multirow[t]{2}{*}{.02} & 5.38 & 5.42 & \multirow[t]{2}{*}{.80} \\
\hline & SD & 1.01 & 1.00 & & 0.71 & 0.64 & \\
\hline \multirow[t]{2}{*}{ High $\mathrm{Hz}$} & Mean & 3.98 & 4.14 & \multirow[t]{2}{*}{.45} & 4.23 & 4.29 & \multirow[t]{2}{*}{.75} \\
\hline & $\mathrm{SD}$ & 1.19 & 1.12 & & 0.96 & 1.06 & \\
\hline $\mathrm{Lo} / \mathrm{Hi}$ & Mean & 1.28 & 1.33 & \multirow[t]{2}{*}{$\uparrow .43$} & 1.32 & 1.33 & \multirow[t]{2}{*}{$\uparrow .76$} \\
\hline Ratio & $\mathrm{SD}$ & 0.21 & 0.17 & & 0.23 & 0.29 & \\
\hline \multicolumn{8}{|c|}{ Time Domains } \\
\hline \multirow[t]{2}{*}{ pNN50 } & Mean & 3.25 & 3.39 & \multirow[t]{2}{*}{.69} & 4.35 & 5.72 & \multirow[t]{2}{*}{.11} \\
\hline & $\mathrm{SD}$ & 3.72 & 3.77 & & 4.19 & 6.19 & \\
\hline \multirow[t]{2}{*}{ rMSSD } & Mean & 19.50 & 20.17 & \multirow[t]{2}{*}{.07} & 21.83 & 23.50 & \multirow[t]{2}{*}{.27} \\
\hline & $\mathrm{SD}$ & 8.40 & 9.21 & & 8.20 & 11.18 & \\
\hline \multirow[t]{2}{*}{ SD } & Mean & 36.17 & 39.08 & \multirow[t]{2}{*}{.53} & 39.83 & 39.83 & \multirow[t]{2}{*}{1.00} \\
\hline & SD & 14.21 & 13.91 & & 12.42 & 12.04 & \\
\hline \multirow[t]{2}{*}{ SDNN } & Mean & 97.17 & 93.08 & \multirow[t]{2}{*}{$\downarrow .04$} & 106.67 & 103.67 & \multirow[t]{2}{*}{$\downarrow .56$} \\
\hline & SD & 30.36 & 24.72 & & 23.99 & 29.72 & \\
\hline \multirow[t]{2}{*}{ SDANN } & Mean & 89.17 & 82.17 & \multirow[t]{2}{*}{$\downarrow .23$} & 97.19 & 95.00 & \multirow[t]{2}{*}{$\downarrow .70$} \\
\hline & $\mathrm{SD}$ & 27.21 & 22.34 & & 22.78 & 29.87 & \\
\hline
\end{tabular}

${ }^{1}$ Paired t-Test: $\quad$ Significant at $\mathrm{p}=\leq .05$

Trend at $\mathrm{p}=\leq .10>.05$ 
Table 31

Differences Between Pre- and Post-Test Mean Values for Heart Rate Variability for Postmenopausal Women By Age at Menopause

\begin{tabular}{|c|c|c|c|c|c|c|c|}
\hline \multirow{3}{*}{\multicolumn{2}{|c|}{ HRV Variables }} & \multicolumn{6}{|c|}{ Age at Menopause } \\
\hline & & \multicolumn{3}{|c|}{$\geq 51$ years $(n=7)$} & \multicolumn{3}{|c|}{$<51$ years $(n=17)$} \\
\hline & & Pre-Test & Post-Test & $\mathrm{p}^{1}$ & Pre-Test & Post-Test & $\mathrm{p}^{1}$ \\
\hline \multicolumn{8}{|c|}{ Frequency Domains } \\
\hline \multirow[t]{2}{*}{ Total Hz } & Mean & 6.31 & 6.32 & \multirow[t]{2}{*}{.96} & 6.19 & 6.41 & \multirow[t]{2}{*}{.05} \\
\hline & $\mathrm{SD}$ & 0.84 & 0.71 & & 0.80 & 0.83 & \\
\hline \multirow[t]{2}{*}{ Low Hz } & Mean & 5.18 & 5.35 & \multirow{2}{*}{.62} & 5.14 & 5.41 & \multirow[t]{2}{*}{.03} \\
\hline & $\mathrm{SD}$ & 0.97 & 0.70 & & 0.88 & 0.89 & \\
\hline \multirow{2}{*}{ High Hz } & Mean & 4.29 & 4.13 & \multirow{2}{*}{$\downarrow .62$} & 4.03 & 4.25 & \multirow{2}{*}{.11} \\
\hline & SD & 1.26 & 1.12 & & 1.01 & 1.08 & \\
\hline $\mathrm{Lo} / \mathrm{Hi}$ & Mean & 1.28 & 1.35 & \multirow{2}{*}{$\uparrow .33$} & 1.31 & 1.32 & \multirow{2}{*}{$\uparrow .85$} \\
\hline Ratio & SD & 0.30 & 0.26 & & 0.19 & 0.23 & \\
\hline \multicolumn{8}{|c|}{ Time Domains } \\
\hline \multirow[t]{2}{*}{ pNN50 } & Mean & 3.33 & 4.27 & \multirow{2}{*}{.41} & 3.99 & 4.67 & \multirow[t]{2}{*}{.17} \\
\hline & SD & 2.16 & 3.73 & & 4.49 & 5.73 & \\
\hline \multirow[t]{2}{*}{ rMSSD } & Mean & 20.14 & 21.57 & \multirow[t]{2}{*}{.53} & 20.88 & 21.94 & \multirow[t]{2}{*}{.26} \\
\hline & $\mathrm{SD}$ & 6.15 & 8.32 & & 9.08 & 11.06 & \\
\hline \multirow[t]{2}{*}{ SD } & Mean & 37.71 & 40.14 & \multirow[t]{2}{*}{.46} & 38.12 & 39.18 & \multirow[t]{2}{*}{.42} \\
\hline & $\mathrm{SD}$ & 11.84 & 12.23 & & 14.04 & 13.29 & \\
\hline \multirow[t]{2}{*}{ SDNN } & Mean & 93.00 & 88.86 & \multirow[t]{2}{*}{$\downarrow .48$} & 105.59 & 102.29 & \multirow[t]{2}{*}{$\downarrow .36$} \\
\hline & SD & 16.16 & 21.77 & & 31.84 & 28.91 & \\
\hline \multirow[t]{2}{*}{ SDANN } & Mean & 83.71 & 77.14 & \multirow{2}{*}{$\downarrow .27$} & 97.06 & 93.29 & \multirow[t]{2}{*}{$\downarrow .37$} \\
\hline & SD & 14.94 & 18.36 & & 27.40 & 28.47 & \\
\hline
\end{tabular}

${ }^{1}$ Paired t-Test: $\quad$ Significant at $\mathrm{p}=\leq .05 \quad$ Trend at $\mathrm{p}=\leq .10>.05$ 
Differences Between Pre- and Post-Test HRV in Black Women

Because of the significant differences in mean values of the frequency domains between pre- and post-tests in the Black women (see Table 11), further analyses were done on specific variables within that population. Tables 32 through 35 summarize the changes found using paired $t$-tests to examine differences between the means across the variables of presence/absence of hypertension, smoking status, exercise status, and years of menopause. 
Table 32

Differences Between Pre- and Post-Test Mean Values for Heart Rate Variability for Black Postmenopausal Women Across and Between the Absence/Presence of Hypertension

\begin{tabular}{|c|c|c|c|c|c|c|c|}
\hline \multirow{3}{*}{\multicolumn{2}{|c|}{ HRV Variables }} & \multicolumn{6}{|c|}{ Medical Variables } \\
\hline & & \multicolumn{3}{|c|}{ Hypertension-Absent $(n=6)$} & \multicolumn{3}{|c|}{ Hypertension-Present $(\mathrm{n}=9)$} \\
\hline & & Pre-Test & Post-Test & $\mathrm{p}^{1}$ & Pre-Test & Post-Test & \\
\hline \multicolumn{8}{|c|}{ Frequency Domains } \\
\hline \multirow[t]{2}{*}{ Total Hz } & Mean & 6.48 & 6.78 & \multirow[t]{2}{*}{.12} & 5.98 & 6.31 & \multirow[t]{2}{*}{.08} \\
\hline & $\mathrm{SD}$ & 0.72 & 0.68 & & 0.90 & 0.58 & \\
\hline \multirow[t]{2}{*}{ Low Hz } & Mean & 5.49 & 5.82 & \multirow[t]{2}{*}{.12} & 4.92 & 5.24 & \multirow[t]{2}{*}{.14} \\
\hline & $\mathrm{SD}$ & 0.81 & 0.75 & & 0.91 & 0.57 & \\
\hline \multirow[t]{2}{*}{ High Hz } & Mean & 4.34 & 4.58 & \multirow[t]{2}{*}{.21} & 3.89 & 4.29 & \multirow[t]{2}{*}{.04} \\
\hline & $\mathrm{SD}$ & 0.97 & 0.93 & & 1.17 & 0.94 & \\
\hline $\mathrm{Lo} / \mathrm{Hi}$ & Mean & 1.29 & 1.29 & \multirow[t]{2}{*}{.91} & 1.32 & 1.26 & \multirow[t]{2}{*}{$\downarrow .09$} \\
\hline Ratio & $\mathrm{SD}$ & 0.13 & 0.12 & & 0.23 & 0.23 & \\
\hline \multicolumn{8}{|c|}{ Time Domains } \\
\hline \multirow[t]{2}{*}{ pNN50 } & Mean & 4.33 & 3.75 & \multirow[t]{2}{*}{$\downarrow .33$} & 3.20 & 4.96 & \multirow[t]{2}{*}{.03} \\
\hline & SD & 4.45 & 4.66 & & 3.82 & 4.98 & \\
\hline \multirow[t]{2}{*}{ rMSSD } & Mean & 22.83 & 22.67 & \multirow[t]{2}{*}{$\downarrow .92$} & 18.44 & 21.78 & \multirow[t]{2}{*}{.006} \\
\hline & SD & 8.70 & 9.83 & & 7.99 & 8.98 & \\
\hline \multirow[t]{2}{*}{ SD } & Mean & 41.67 & 43.67 & \multirow[t]{2}{*}{039} & 35.44 & 38.00 & \multirow[t]{2}{*}{.23} \\
\hline & SD & 12.77 & 11.36 & & 16.34 & 12.57 & \\
\hline \multirow[t]{2}{*}{ SDNN } & Mean & 109.83 & 107.67 & \multirow[t]{2}{*}{$\downarrow .69$} & 100.00 & 95.56 & \multirow[t]{2}{*}{$\downarrow .44$} \\
\hline & $\mathrm{SD}$ & 32.42 & 29.26 & & 33.45 & 32.86 & \\
\hline \multirow[t]{2}{*}{ SDANN } & Mean & 99.50 & 99.33 & \multirow[t]{2}{*}{$\downarrow .98$} & 92.44 & 85.44 & \multirow[t]{2}{*}{$\downarrow .23$} \\
\hline & SD & 31.15 & 29.71 & & 30.03 & 31.95 & \\
\hline
\end{tabular}

${ }^{1}$ Paired t-Test: $\quad$ Significant at $\mathrm{p}=\leq .05 \quad$ Trend at $\mathrm{p}=\leq .10>.05$ 
Table 33

Differences Between Pre- and Post-Test Mean Values for Heart Rate Variability for Black Postmenopausal Women Across and Between Smoking Status

\begin{tabular}{|c|c|c|c|c|c|c|c|}
\hline \multirow{3}{*}{\multicolumn{2}{|c|}{ HRV Variables }} & \multicolumn{6}{|c|}{ Medical Variables } \\
\hline & & \multicolumn{3}{|c|}{ Non-Smoker $(\mathrm{n}=10)$} & \multicolumn{3}{|c|}{ Smoker $(\mathrm{n}=5)$} \\
\hline & & Pre-Test & Post-Test & $\mathrm{p}^{1}$ & Pre-Test & Post-Test & $\mathrm{p}^{1}$ \\
\hline \multicolumn{8}{|c|}{ Frequency Domains } \\
\hline \multirow[t]{2}{*}{ Total Hz } & Mean & 6.29 & 6.58 & \multirow[t]{2}{*}{.09} & 5.98 & 6.33 & \multirow[t]{2}{*}{.11} \\
\hline & $\mathrm{SD}$ & 0.77 & 0.48 & & 1.03 & 0.94 & \\
\hline \multirow[t]{2}{*}{ Low $\mathrm{Hz}$} & Mean & 5.27 & 5.57 & \multirow[t]{2}{*}{.12} & 4.92 & 5.29 & \multirow[t]{2}{*}{.18} \\
\hline & $\mathrm{SD}$ & 0.79 & 0.48 & & 1.14 & 1.04 & \\
\hline \multirow[t]{2}{*}{$\mathrm{High} \mathrm{Hz}$} & Mean & 4.23 & 4.58 & \multirow[t]{2}{*}{.06} & 3.75 & 4.05 & \multirow[t]{2}{*}{.11} \\
\hline & $\mathrm{SD}$ & 0.93 & 0.57 & & 1.41 & 1.40 & \\
\hline $\mathrm{Lo} / \mathrm{Hi}$ & Mean & 1.27 & 1.23 & \multirow[t]{2}{*}{$\downarrow .20$} & 1.38 & 1.36 & \multirow[t]{2}{*}{$\downarrow .19$} \\
\hline Ratio & SD & 0.15 & 0.12 & & 0.26 & 0.28 & \\
\hline \multicolumn{8}{|c|}{ Time Domains } \\
\hline \multirow[t]{2}{*}{ pNN50 } & Mean & 3.51 & 4.59 & \multirow[t]{2}{*}{.22} & 3.94 & 4.24 & \multirow[t]{2}{*}{.13} \\
\hline & $\mathrm{SD}$ & 3.29 & 4.58 & & 5.53 & 5.54 & \\
\hline \multirow[t]{2}{*}{ rMSSD } & Mean & 20.40 & 22.10 & \multirow[t]{2}{*}{.25} & 19.80 & 22.20 & \multirow[t]{2}{*}{.009} \\
\hline & $\mathrm{SD}$ & 6.54 & 7.28 & & 11.97 & 12.79 & \\
\hline \multirow[t]{2}{*}{ SD } & Mean & 39.10 & 40.90 & \multirow[t]{2}{*}{.35} & 35.60 & 39.00 & \multirow[t]{2}{*}{.22} \\
\hline & $\mathrm{SD}$ & 14.26 & 11.29 & & 17.47 & 14.71 & \\
\hline \multirow[t]{2}{*}{ SDNN } & Mean & 102.10 & 102.80 & \multirow[t]{2}{*}{.88} & 107.60 & 95.60 & \multirow[t]{2}{*}{$\downarrow .08$} \\
\hline & $\mathrm{SD}$ & 31.61 & 31.98 & & 36.95 & 31.89 & \\
\hline \multirow[t]{2}{*}{ SDANN } & Mean & 92.40 & 93.90 & \multirow[t]{2}{*}{.77} & 101.00 & 85.20 & \multirow[t]{2}{*}{$\downarrow .02$} \\
\hline & SD & 29.61 & 32.49 & & 32.04 & 29.66 & \\
\hline
\end{tabular}

${ }^{1}$ Paired t-Test: $\quad$ Significant at $\mathrm{p}=\leq .05 \quad$ Trend at $\mathrm{p}=\leq .10>.05$ 
Table 34

Differences Between Pre- and Post-Test Mean Values for Heart Rate Variability for Black Postmenopausal Women Across and Between Exercise Status

\begin{tabular}{|c|c|c|c|c|c|c|c|}
\hline \multirow{3}{*}{\multicolumn{2}{|c|}{ HRV Variables }} & \multicolumn{6}{|c|}{ Medical Variables } \\
\hline & & \multicolumn{3}{|c|}{ Non-Exerciser $(\mathrm{n}=8)$} & \multicolumn{3}{|c|}{ Exerciser $(\mathrm{n}=7)$} \\
\hline & & Pre-Test & Post-Test & $\mathrm{p}^{1}$ & Pre-Test & Post-Test & $\mathrm{p}^{1}$ \\
\hline \multicolumn{8}{|c|}{ Frequency Domains } \\
\hline \multirow[t]{2}{*}{ Total Hz } & Mean & 6.22 & 6.47 & .11 & 6.14 & 6.52 & .09 \\
\hline & $\mathrm{SD}$ & 0.87 & 0.73 & & 0.88 & 0.58 & \\
\hline \multirow[t]{2}{*}{ Low $\mathrm{Hz}$} & Mean & 5.18 & 5.40 & .23 & 5.12 & 5.55 & .09 \\
\hline & SD & 0.95 & 0.81 & & 0.89 & 0.58 & \\
\hline \multirow[t]{2}{*}{ High Hz } & Mean & 4.11 & 4.34 & .05 & 4.03 & 4.48 & .09 \\
\hline & SD & 1.21 & 1.18 & & 1.02 & 0.55 & \\
\hline $\mathrm{Lo} / \mathrm{Hi}$ & Mean & 1.32 & 1.29 & $\downarrow .22$ & 1.30 & 1.25 & $\downarrow .26$ \\
\hline Ratio & SD & 0.23 & 0.25 & & 0.16 & 0.09 & \\
\hline \multicolumn{8}{|c|}{ Time Domains } \\
\hline \multirow[t]{2}{*}{ pNN50 } & Mean & 3.76 & 4.98 & .19 & 3.53 & 3.90 & .62 \\
\hline & $\mathrm{SD}$ & 4.23 & 4.94 & & 3.97 & 4.78 & \\
\hline \multirow[t]{2}{*}{ rMSSD } & Mean & 20.38 & 23.50 & .02 & 20.00 & 20.57 & .69 \\
\hline & SD & 9.15 & 10.62 & & 7.87 & 7.18 & \\
\hline \multirow[t]{2}{*}{$\mathrm{SD}$} & Mean & 37.63 & 41.25 & .04 & 38.29 & 39.14 & .75 \\
\hline & $\mathrm{SD}$ & 13.53 & 11.74 & & 17.36 & 13.18 & \\
\hline \multirow[t]{2}{*}{ SDNN } & Mean & 110.75 & 108.75 & $\downarrow .74$ & 96.14 & 90.86 & $\downarrow .33$ \\
\hline & $\mathrm{SD}$ & 31.27 & 33.57 & & 33.95 & 26.95 & \\
\hline \multirow[t]{2}{*}{ SDANN } & Mean & 103.63 & 98.88 & $\downarrow .46$ & 85.71 & 82.00 & $\downarrow .57$ \\
\hline & SD & 28.60 & 33.41 & & 29.79 & 27.07 & \\
\hline
\end{tabular}

${ }^{1}$ Paired t-Test: $\quad$ Significant at $\mathrm{p}=\leq .05$

Trend at $\mathrm{p}=\leq .10>.05$ 
Table 35

Differences Between Pre- and Post-Test Mean Values for Heart Rate Variability for Black Postmenopausal Women Across and Between Years of Menopause

HRV Variables $\quad$ Years of Menopause

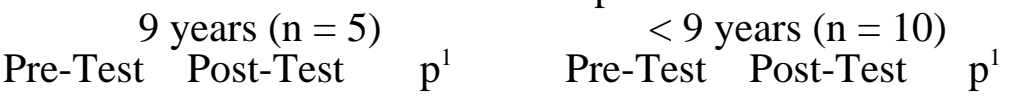

Frequency Domains

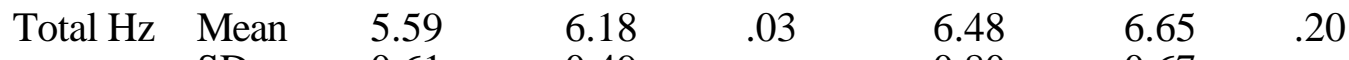

$\begin{array}{lllll}\text { SD } & 0.61 & 0.49 & 0.80 & 0.67\end{array}$

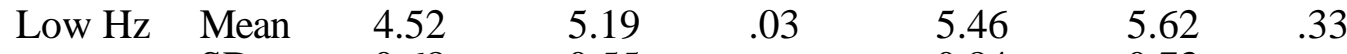

$\begin{array}{lllll}\text { SD } & 0.68 & 0.55 & 0.84 & 0.73\end{array}$

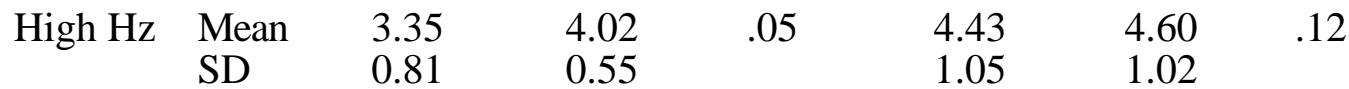

$\begin{array}{llllllll}\text { Lo/Hi } & \text { Mean } & 1.38 & 1.29 & \downarrow .16 & 1.27 & 1.26 & \downarrow .53 \\ \text { Ratio } & \text { SD } & 0.17 & 0.13 & & 0.20 & 0.22 & \end{array}$

Time Domains

$\begin{array}{llcccccc}\text { pNN50 } & \text { Mean } & 1.02 & 1.90 & .05 & 4.97 & 5.76 & .36 \\ & \text { SD } & 1.06 & 1.46 & & 4.26 & 5.31 & \\ \text { rMSSD } & \text { Mean } & 14.40 & 16.80 & .0006 & 23.10 & 24.80 & .25 \\ & \text { SD } & 4.28 & 4.27 & & 8.39 & 9.70 & \\ \text { SD } & \text { Mean } & 27.60 & 32.40 & .03 & 43.10 & 44.20 & .58 \\ & \text { SD } & 5.86 & 8.56 & & 15.47 & 11.87 & \\ \text { SDNN } & \text { Mean } & 86.80 & 83.60 & \downarrow .60 & 112.50 & 108.80 & \downarrow .49 \\ & \text { SD } & 18.38 & 19.74 & & 34.96 & 32.94 & \\ \text { SDANN } & \text { Mean } & 81.60 & 77.80 & \downarrow .67 & 102.10 & 97.60 & \downarrow .39 \\ & \text { SD } & 18.85 & 23.40 & & 32.36 & 32.96 & \end{array}$

${ }^{1}$ Paired t-Test: $\quad$ Significant at $\mathrm{p}=\leq .05 \quad$ Trend at $\mathrm{p}=\leq .10>.05$ 


\section{VITA}

Diane Todd Pace graduated with a diploma in nursing from Methodist Hospital School of Nursing, Memphis, Tennessee, December, 1971. She received a Bachelor's Degree in Health Advisory Services from Arkansas State University, Jonesboro, Arkansas, in December, 1982, and graduated Magna Cum Laude with a Master of Science Degree in Education from University of Memphis, August, 1984. In May of 1995, she graduated Summa Cum Laude from Union University with a Bachelor of Science in Nursing. Upon graduation, Mrs. Pace entered the doctoral program in nursing at the University of Tennessee, Memphis and completed requirements for a Master of Science Degree in Nursing with a concentration of study in the Family Nurse Practitioner Program in June, 1996. She is currently a PhD candidate in Nursing with expected graduation in June, 1998.

Mrs. Pace is a member of Alpha Chi and Phi Kappa Phi Honor Societies. Additionally, she is a member, past vice president, and recipient of the first nursing scholarship for Nu Lambda Chapter of Sigma Theta Tau Nursing Honor Society. She has been named to Who's Who in American Colleges and Universities, Who's Who in Nursing, Outstanding Young Women of America, and was one of the first recipients of The Great 100 Nurses celebration in Dallas, Texas. She is a member of the American and Tennessee Nurses Association, Greater Memphis Advanced Practice Council, Southern Nursing Research Society, and the North America Menopause Society. She has a Volunteer Faculty appointment through UTM College of Nursing and affiliation as a clinical preceptor through The University of Memphis. She has presented her research at various conferences and is developing a program of research in women's health.

Mrs. Pace has been employed in a variety of health care roles including clinician, nursing faculty, manager, and consultant. Currently, she is certified as a Family Nurse Practitioner with active licensure and prescriptive authority through the Tennessee State Board of Nursing and is employed full-time at two community-based healthcare clinics. 